\author{
Universidade de São Paulo \\ Instituto de Física
}

\title{
Modelos unidimensionais na rede com inspiração em biomembranas: pseudo-transições e quase-fases
}

\author{
Tiago Ferreira Lourenço
}

Orientadora: Prof ${ }^{\mathrm{a}}$. Dr ${ }^{\mathrm{a}}$. Vera Bohomoletz Henriques

Dissertação de mestrado apresentada ao Instituto de Física da Universidade de São Paulo, como requisito parcial para a obtenção do título de Mestre em Ciências.

Banca Examinadora:

Prof $^{a}$. Dr ${ }^{\mathrm{a}}$. Vera Bohomoletz Henriques - Orientadora (IFUSP)

Prof. Dr. Onofre Rojas Santos (UFLA)

Prof. Dr. Mario Noboru Tamashiro (UNICAMP) 
FICHA CATALOGRÁFICA

Preparada pelo Serviço de Biblioteca e Informação do Instituto de Física da Universidade de São Paulo

Lourenço, Tiago Ferreira

Modelos unidimensionais na rede com inspiração em biomembranas: pseudo-transições e quase-fases. São Paulo, 2021.

Dissertação (Mestrado) - Universidade de São Paulo. Instituto de Física. Depto. de Física Geral.

Orientador: Profa. Dra. Vera Bohomoletz Henriques

Área de Concentração: Física

Unitermos: 1. Mecânica estatística; 2. Modelos estatísticos na rede; 3. Modelos unidimensionais,.

USP/IF/SBI-063/2021 
University of São Paulo

Physics Institute

\title{
Linear lattice models inspired by biomembranes: pseudo-transitions and quasi-phases
}

\author{
Tiago Ferreira Lourenço
}

Supervisor: Prof ${ }^{a}$. Dr ${ }^{\mathrm{a}}$. Vera Bohomoletz Henriques

Dissertation submitted to the Physics Institute of the University of São Paulo in partial fulfillment of the requirements for the degree of Master of Science.

Examining Committee:

Prof $^{a}$. Dr ${ }^{a}$. Vera Bohomoletz Henriques - Supervisor (IFUSP)

Prof. Dr. Onofre Rojas Santos (UFLA)

Prof. Dr. Mario Noboru Tamashiro (UNICAMP) 

À minha mãe, Cláudia. 



\section{Agradecimentos}

Muitas pessoas cabem aqui, não necessariamente por contribuições diretas à pesquisa, mas por terem marcado, e continuam marcando, as demais dimensões que formam a totalidade da minha vida. Antes de tudo, somos seres sociais, inseridos em um contexto espaço-temporal, sob condicionantes materiais e ideológicos que brotam das relações concretas e antagônicas na sociedade de classes. Ninguém rompe com esses condicionantes sozinho, a transformação, mesmo no sentido individual, é parte de um processo coletivo. Devo minha gratidão a todas e todos que fizeram e fazem parte desse processo.

À professora Vera, por toda a paciência, atenção, ensinamentos, reflexões e inspiração. Por me ajudar a crescer, não apenas nas questões relacionadas à física, mas também as que passam por política, educação e sociedade, tão importantes quanto. É um exemplo de cientista que sabe que a produção do conhecimento não é uma atividade humana pairando sobre as contradições do mundo, como se nada tivesse a ver com elas. Uma intelectual orgânica da classe trabalhadora, que também luta por tempos melhores para todas e todos. Um dos seres humanos mais inteligentes e sensíveis que já conheci, espero ser seu orientando para o resto da vida! Agradeço também ao Jozi pelas conversas, sugestões e auxílio, essenciais no desenvolvimento do trabalho.

À minha mãe, Cláudia, e ao meu pai, Marcelo, que mesmo com todas as dificuldades nunca me faltaram com apoio, e apesar da distância sempre estiveram presentes. As minhas palavras nunca vão ser suficientes para demonstrar o tamanho do meu amor, orgulho e gratidão. Às minhas tias Clara, Cleide e Cristina, cuja ajuda foi fundamental, vou passar a vida toda tentando retribuir, mas sei que nunca vou chegar perto de conseguir. À vó Ana, obrigado por me ensinar tanto, com a sua bondade impossível de descrever e a sua simplicidade carregada de sabedoria. 
À minha companheira, Laura, por todo o carinho e parceria nesses anos. Sou imensamente grato por compartilhar a existência com você, o caminho é mais fácil de percorrer segurando a sua mão. À Viviani e à Saada, pelo suporte nos momentos difíceis e pelo acolhimento, por permitir e me fazer sentir parte da família.

Aos camaradas da militância, educadoras e educadores do Cursinho Popular Laudelina de Campos Melo, em especial: Victor, Lívia, Lúcia, Bia, Cinthia e Carol, pela amizade, pela coragem, pelas reflexões e debates. Vocês são essenciais na minha formação como ser humano, e sempre conseguem converter a atmosfera de desesperança, que com frequência o sistema nos submerge, em combustível para a luta.

Às amizades de tempos, saudosas nessa difícil época de pandemia: Boku e Alessandra por sempre me salvarem - e não foram poucas vezes. Carol pela ajuda com latex, python e mathematica. André, Jéssica, Igor, Bruno, Barreira e Tobias. Obrigado a todas e todos, sei que vocês estão por mim como eu estou por vocês. Se nós somos a média aritmética das pessoas que escolhemos manter próximas, então eu posso ficar feliz por quem eu sou.

É incontornável expressar minha gratidão às trabalhadoras e trabalhadores da Universidade de São Paulo, especialmente àquelas dos serviços básicos: a equipe de limpeza, porteiras e porteiros, seguranças, técnicas e técnicos, bibliotecárias e bibliotecários, entre tantos outros. Todas as pessoas responsáveis por trabalhos muitas vezes invisíveis e quase sempre desvalorizados, porém fundamentais para o coletivo. São vocês que fazem tudo funcionar.

Por fim, à memória da vó Maria, que o tempo levou antes mesmo de me ver na faculdade. Trago nas lembranças e no coração os momentos e ensinamentos que ajudaram a moldar quem eu sou hoje. É um privilégio ter crescido sob os seus cuidados. 

"A teoria materialista de que os homens são produtos das circunstâncias e da educação e de que, portanto, homens modificados são produtos de circunstâncias diferentes e de educação modificada, esquece que as circunstâncias são modificadas precisamente pelos homens e que o próprio educador precisa ser educado. Leva, pois, forçosamente, à divisão da sociedade em duas partes, uma das quais se sobrepõe à sociedade [...]. A coincidência da modificação das circunstâncias e da atividade humana só pode ser apreendida e racionalmente compreendida como prática transformadora." 



\section{Resumo}

Membranas biológicas são as barreiras que separam o interior do exterior das células dos animais, além promoverem diversos processos bioquímicos responsáveis pela regulação da composição intracelular e a comunicação intercelular. Estudos sobre as propriedades físicas das membranas buscando a melhor compreensão das suas funções biológicas têm atraído cada vez mais atenção. Os principais componentes desses sistemas são os lipídios, moléculas anfifílicas, isto é, feitas de duas porções: uma cabeça polar e caudas apolares. O efeito hidrofóbico combinado com a interação eletrostática induz a agregação e formação de estruturas estáveis. Experimentos feitos com membranas modelo sugerem a existência de fases termodinâmicas distintas, onde ocorrem transições sob variação de determinados parâmetros, como temperatura e pressão lateral. Os modelos simplificados na rede são importantes ferramentas para a descrição desses sistemas. O mais conhecido é o modelo de Doniach, que possui dois estados que traduzem a configuração das caudas apolares, enquanto esticadas ou enquanto dobradas, esta última possuindo degenerescência. Recentemente, Guidi e Henriques propuseram uma modificação no modelo: o fluido de rede de Doniach (DLG), por meio da adição de um estado representando um buraco na rede, possibilitando descrever flutuações na densidade. O DLG possui solução obtida por aproximação de campo médio e por simulações computacionais. Neste trabalho, trataremos de obter a solução exata para o caso unidimensional, através do método da matriz de transferência, e lançaremos um olhar sobre as propriedades do seu diagrama de fases. Concomitantemente, desenvolveremos algumas ideias relacionadas ao fenômeno das pseudo-transições e quase-fases, termos que se referem a mudanças abruptas, mas contínuas, nas propriedades do sistema devido à variação de temperatura, manifestados na primeira derivada da energia livre, e acompanhadas de picos na segunda derivada, características de transições descontínuas e contínuas, respectivamente. Não obstante, essas quase-fases não são separadas por linhas onde a energia livre perde a analiticidade, de sorte que não se tratam de transições de fase formais (daí vem a sugestiva terminologia). Por fim, salientamos que modelos unidimensionais podem nos dar pistas valiosas sobre o comportamento dos mesmos sistemas em dimensões maiores, como é o caso das pseudo-transições. O conhecido modelo de Ising apresenta tal propriedade, bem como o fluido de rede de Doniach, sinalizando a existência de transições de fase - que de fato ocorrem - nas suas versões de dimensão maior que um.

palavras-chave: mecânica estatística, modelos estatísticos na rede, modelos unidimensionais, modelos estatísticos para biomembranas, pseudo-transições, quase-fases. 



\section{Abstract}

Biological membranes are the barriers that separate the interior from the exterior of animal cells, in addition to promoting several biochemical processes responsible for regulating intracellular composition and intercellular communication. Studies on the physical properties of membranes seeking a better understanding of their biological functions have attracted increasing attention. The main components of these systems are lipids, amphiphilic molecules, that is, made up of two parts: a polar head and non-polar tails. The hydrophobic effect combined with the electrostatic interaction induces the aggregation and formation of stable structures. Experiments carried out with model membranes suggest the existence of distinct thermodynamic phases, where transitions occur under the variation of certain parameters, such as temperature and lateral pressure. The simplified models on the lattice are important tools for the description of these systems. The best known is the Doniach model, which has two states that translate the configuration of the nonpolar tails, while stretched or folded, the latter having degeneracy. Recently, Guidi e Henriques proposed a modification in the model: the Doniach lattice fluid (DLG), through the addition of a state representing a hole in the lattice, making it possible to describe density fluctuations. The DLG has a solution obtained by mean-field approximation and by computational simulations. In this work, we will try to obtain the exact solution for the one-dimensional case, through the transfer-matrix method, and we will look at the properties of its phase diagram. At the same time, we will develop some ideas related to the phenomenon of pseudo-transitions and quasi-phases, terms that refer to abrupt changes, but continuous, in system properties due to temperature variation, manifested in the first derivative of free energy, and accompanied by peaks in the second derivative, characteristics of discontinuous and continuous transitions, respectively. Nevertheless, these quasi-phases are not separated by lines where the free energy loses its analyticity, so they are not formal phase transitions (hence the suggestive terminology). Finally, we emphasize that one-dimensional models can give us valuable clues about the behavior of the same systems in larger dimensions, such as it is the case of the pseudo-transitions. The well-known Ising model has such a property, as well as the Doniach lattice fluid, signaling the existence of phase transitions - which actually occur — in its larger-than-one dimensional versions.

keywords: statistical mechanics, statistical lattice models, one-dimensional models, biomembranes lattice models, pseudo-transitions, quasi-phases. 



\section{Sumário}

1 Introdução 1

2 Biomembranas e modelos 9

2.1 Lipídios, membranas e modelos experimentais . . . . . . . . . . . . . . 9

2.2 O modelo de Doniach - dois estados . . . . . . . . . . . . . . . . . . . 13

2.3 O fluido de rede de Doniach - três estados . . . . . . . . . . . . . . . 15

3 Modelos estatísticos - soluções analíticas 16

3.1 Gás ideal . . . . . . . . . . . . . . . . . . . . . . . . 16

3.1 .1 Na rede .............................. 16

3.1 .2 No contínuo . . . . . . . . . . . . . . . . . . . 19

3.2 Modelos interagentes na rede . . . . . . . . . . . . . . . . 21

3.2.1 Mapeamento do modelo de Ising no fluido de rede . . . . . . . . . . 22

3.3 Aproximação de campo médio . . . . . . . . . . . . . . . . . . 24

3.3.1 Modelo de Ising . . . . . . . . . . . . . . . . . . . 25

3.3.2 Modelo de Ising degenerado . . . . . . . . . . . . . . . . 28

4 Modelos unidimensionais na rede $\quad 33$

4.1 Modelo de Ising unidimensional $\ldots \ldots$. . . . . . . . . . . . . . 33

4.2 Modelo de Ising unidimensional degenerado . . . . . . . . . . . . . . . 39

4.3 Fluido de rede unidimensional $\ldots \ldots \ldots \ldots$

4.4 Fluido de rede unidimensional degenerado . . . . . . . . . . . . . . . . . . 59

5 O fluido de rede de Doniach (DLG) 65 
6 Conclusões e considerações finais

Referências Bibliográficas

A Método da matriz de transferência

B Resolução da equação cúbica 


\section{Capítulo 1}

\section{Introdução}

As membranas biológicas são essenciais para a existência e manutenção da vida, graças a inúmeras funções bioquímicas, como a organização da célula e a permeabilidade seletiva que regula a composição do meio intracelular [1]. Essas características tornam esse sistema um objeto de estudo de interesse em diversas áreas. Os principais componentes das biomembranas são os fosfolipídios - moléculas compostas por uma cabeça hidrofílica, que pode ser neutra ou estar eletricamente carregada, e duas caudas hidrofóbicas - e, em menor quantidade, proteínas. A propriedade anfifílica dos lipídios, combinada com a interação eletrostática, é o que os induz a se organizarem em estruturas, como monocamadas e bicamadas, em solução aquosa ou na interface água-ar. Ademais, identificamos nesses sistemas a existência de distintas fases termodinâmicas, associadas ao estado dos lipídios — que por sua vez é caracterizado pela conformação das caudas apolares. Por conseguinte, sob variação de parâmetros como temperatura ou pressão lateral, observamos o fenômeno das transições de fase.

Para esse estudo nos apoiaremos matematicamente nos modelos de rede, tomando como base o modelo de Ising $[2,3,4,5]$ : sistema ferromagnético de spin 1/2, proposto por Wilhelm Lenz ao seu orientando de doutorado Ernst Ising, em 1920, que, em sua tese, o resolveu exatamente para o caso unidimensional. A solução exata para duas dimensões, na ausência de campo externo, foi obtida por Lars Onsager [6], em 1944. Ainda hoje, a solução exata para o problema na presença de campo externo e em três dimensões permanece uma tarefa incompleta. Quando dizemos que a nossa base será o modelo de Ising, significa, por um lado, que a estrutura matemática dos nossos sistemas de interesse é análoga ao dele: o fluido de rede, por exemplo, é 
um modelo de dois estados — tal como Ising de spin 1/2 —, mas cuja descrição é feita através de variáveis de ocupação - enquanto Ising usa de variáveis de spin. A equivalência entre os modelos anui ao mapeamento dos resultados de um no outro, quer dizer, podemos fazer a leitura de diferentes sistemas físicos, desde que suas traduções matemáticas tenham a mesma estrutura. Essa propriedade de universalidade torna o modelo de Ising uma ferramenta inestimável para o estudo de modelos na rede, já que sua solução exata é conhecida em uma e duas dimensões para campo nulo, além de soluções aproximadas, como é o caso da abordagem de campo médio. Por outro lado, o sistema mais complexo que resolveremos neste trabalho se trata de uma extensão do modelo de Ising para spin 1.

O modelo de Doniach [7], proposto por Sebastian Doniach, em 1978, é também um sistema de dois estados, sendo um deles degenerado. É uma das principais ferramentas utilizadas na descrição de biomembranas modelo - estruturas produzidas em laboratório, formadas por um único tipo de lipídio. A descrição de Doniach trata a membrana como uma rede bidimensional, onde cada sítio é ocupado por um lipídio que pode estar em um estado ordenado, dito all-trans, cujas caudas apolares estão esticadas, ou num estado desordenado, cujas caudas apresentam dobras, sendo este último altamente degenerado, pois pretende representar a arbitrariedade das possíveis configurações.
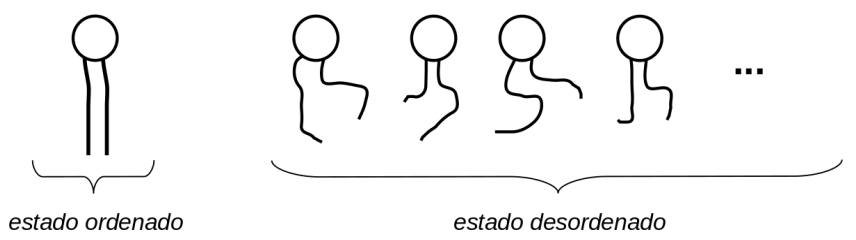

Figura 1.1: Representação dos estados dos lipídios no modelo de Doniach. O estado com as caudas apolares desordenadas é altamente degenerado.

Além dessa classe de sistemas de dois estados, mapeáveis no modelo de Ising de spin 1/2, é possível definir modelos com uma quantidade maior de estados. Nos limitaremos a nos debruçar sobre um caso com três estados, equivalente ao ferromagneto de Ising de spin 1 - cujas variáveis assumem os valores $-1,0$ e 1 . Recentemente, Guidi e Henriques [8, 9] propuseram uma modificação no modelo de Doniach, de sorte a considerar flutuações na densidade da membrana. Isso foi feito a partir da adição de um terceiro estado, o qual descreve um sítio vazio na rede. Esse sistema foi chamado fluido de rede de Doniach (DLG) e será analisado em detalhes 
durante o trabalho.

Nosso estudo foca nos modelos em uma dimensão, cuja resolução será feita através do método da matriz de transferência. O prisma da unidimensionalidade, embora simplificado, é importante para a investigação de vários sistemas físicos, já que a formalização matemática muitas vezes permite a obtenção de soluções exatas, como é o caso dos modelos discretos mencionados. Não obstante, esses modelos unidimensionais podem nos dar pistas importantes sobre os fenômenos que ocorrem em sistemas de dimensões mais altas.

\section{Transições de fase em modelos unidimensionais}

Uma questão controversa relacionada aos modelos em uma dimensão é a existência transições de fase. Apesar de amplamente difundida a ideia da sua impossibilidade nessa abordagem, principalmente devido ao argumento heurístico de Landau [10, 11], é possível encontrar exemplos onde esse fenômeno ocorre, como o modelo de Kittel [12], o modelo de Chui-Weeks [13], o modelo de Dauxois-Peyrard [14] e o modelo de Potts com estados invisíveis [15].

\section{Argumento de Landau}

Considere o estado fundamental de uma cadeia de Ising com $N+1$ sítios, cujos spins estão, digamos, para cima.

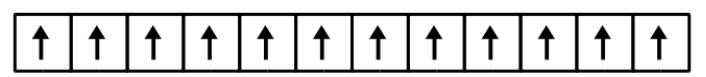

Figura 1.2: Ilustração esquemática de uma rede unidimensional ordenada, com todos os spins apontando para cima.

A energia interna desse sistema é $U_{1}=-N J$ e a entropia é $S_{1}=k_{B} \ln 2$, onde $k_{B}$ é a constante de Boltzmann, já que há duas possibilidades de o sistema estar ordenado — todos os spins para baixo $\left(\sigma_{i}=-1, \forall i\right)$ ou todos para cima $\left(\sigma_{i}=+1, \forall i\right)$. Vamos definir a energia livre desse sistema com um único domínio de $F_{1}$, lembrando que $F=U-T S$, temos

$$
F_{1}=-N J-k_{B} T \ln 2
$$


Agora suponha que escolhemos ao acaso um sítio, e todos os outros à direita deste trocam de estado, criando dois domínios ordenados, como na figura (1.3).

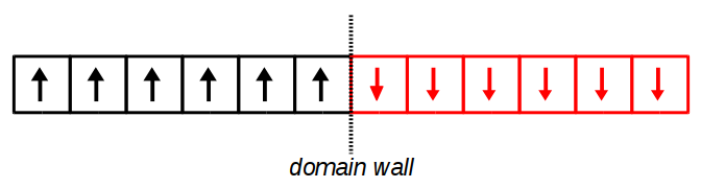

Figura 1.3: Ilustração esquemática de uma rede unidimensional ordenada, com todos os spins apontando para cima no lado esquerdo e todos os spins apontando para baixo no lado direito.

Agora, a energia interna do sistema com dois domínios é dada por $U_{2}=-N J+2 J$, pois o surgimento dessa parede de separação entre os domínios têm um custo energético de $2 J$. Além disso, a entropia do sistema passa a ser $S_{2}=k_{B} \ln (2 N)$, de tal forma que a energia livre será

$$
F_{2}=-N J+2 J-k_{B} T \ln (2 N) .
$$

Por fim, vamos avaliar a variação da energia livre devido ao aparecimento de dois domínios

$$
\begin{gathered}
\Delta F=F_{2}-F_{1}=\left[-N J+2 J-k_{B} T \ln (2 N)\right]-\left(-N J-k_{B} T \ln 2\right) \\
\Delta F=2 J-k_{B} T \ln N .
\end{gathered}
$$

$\mathrm{O}$ argumento de Landau se assenta nesse resultado. A ideia é que para $N$ suficientemente grande, e qualquer temperatura não nula, dois ou mais domínios têm sempre como consequência uma diminuição da energia livre, de tal sorte que essa é uma condição favorável ao sistema.

\section{Transições em uma dimensão e o teorema de van Hove}

Além do argumento exposto, há também resultados matematicamente rigorosos sobre a inexistência de transição de fase em modelos unidimensionais, sendo o mais famoso o teorema de van Hove [16]. Um estudo recente realizado por Custa e Sánchez [17] propõe uma generalização desse resultado, analisando as três hipóteses fundamentais sobre o sistema utilizadas na sua demonstração: homogeneidade, deve ser composto de partículas idênticas, ausência de campos externos, o que significa que as interações devem depender somente das distâncias relativas e, 
por fim, caroços duros, quer dizer, as partículas não podem se sobrepor. Cuesta e Sánchez propõe a flexibilização da segunda e terceira restrições, de forma a incluir no alcance do teorema alguns tipos de campo externo, bem como o caso de sistemas com partículas pontuais. Além disso, eles também sublinham um problema que frequentemente emerge em trabalhos que esbarram com a questão das transições em uma dimensão: o teorema de van Hove é evocado como justificativa generalizada para a inexistência do fenômeno nesses modelos, não obstante tenha sido demonstrado sob as condições que elencamos.

O artigo completo é interessante e deixamos como sugestão para quem se interessa no assunto. Neste trabalho, vamos destacar a análise de sistemas com uma quantidade limitada de estados acessíveis, ou melhor, cuja matriz de transferência tem dimensão finita. Nesse sentido, Cuesta e Sánchez dedicam uma seção do artigo para o modelo de Kittel, de 1969, que se assemelha a um zipper em que cada sítio só pode ser aberto se todos os anteriores também estiverem abertos, e o último está sempre fechado. A energia necessária para abrir uma posição qualquer, se todas as anteriores estiverem abertas é $\epsilon<\infty$, mas se essa condição não ocorre então a energia necessária é infinita. Aliás, o estado aberto possui degenerescência, quer dizer que é possível abrir cada sítio de várias maneiras distintas. Este é um exemplo de modelo unidimensional que possui transição à temperatura finita.

A conclusão importante que gostaríamos de fazer uso é que as transições de fase ocorrem na presença de configurações com energias infinitas, e que levam a entradas nulas na matriz de transferência — que são dadas por pesos de Boltzmann. Isso ocorre pois a demonstração do teorema de van Hove se apoia no teorema de Perron-Frobenius, cujo enunciado [18] é o que segue

Teorema 1. Seja A uma matriz quadrada, irredutível e não-negativa, de ordem maior ou igual a 2. Então

a) $O$ maior autovalor de $A, R_{A}$, é estritamente positivo

b) $R_{A}$ é um autovalor algebricamente simples de $A$

c) Existe um único vetor real $x=\left[x_{i}\right]$ tal que $A x=R_{A} x$ e $x_{1}+\ldots+x_{n}=1$; este vetor é positivo

d) Existe um único vetor real $y=\left[y_{i}\right]$ tal que $y^{T} A=R_{A} y^{T}$ e $x_{n} y_{n}=1$; este vetor é positivo. 
As entradas nulas na matriz de transferência permitem a possibilidade dela vir a se tornar redutivel, fugindo do alcance do teorema de Perron-Frobenius, destarte do teorema de van Hove, abrindo espaço para a ocorrência de transições de fase, que se manifestam em decorrência da troca do maior autovalor.

Retornando um pouco ao mencionado modelo de Kittel, sublinhamos que na situação em que o sistema não possui degenerescência, não há sinais de transição. Por conseguinte, o fato de haver estados energeticamente proibidos é condição necessária, mas não suficiente, para se observar o fenômeno nesse contexto.

\section{Pseudo-transições e quase-fases}

Mas é na sugestão da existência da transição de fases que estamos interessados, as pseudotransições, termo usado pela primeira vez por Pavel Timonin [19], em 2011, em um estudo sobre gelo de spin, e se refere às mudanças abruptas nas características do sistema que se manifestam nas primeiras derivadas da energia livre, como a magnetização e a entropia, bem como um máximo acentuado nas segundas derivadas, como o calor específico e a susceptibilidade, características conhecidas de transições de primeira e segunda ordem, respectivamente. Entretanto, apesar da formação de regiões contrastantes no diagrama de "fases", a energia livre não perde a sua analiticidade, de sorte que, a rigor, não há realmente fases separadas por descontinuidades, mas sim o que foi chamado de quase-fases.

O fenômeno das pseudo-transições vem sendo estudado pelo grupo de Onofre Rojas [20, 21, 22, 23, 24], o qual discute que a ocorrência das quase-fases depende dos pesos de Boltzmann da matriz de transferência do sistema. Em [20], Rojas e Souza mostram que certos modelos decorados se caracterizam por possuir entradas da matriz de transferência de fora da diagonal principal muito menores do que as que estão nela. Nesses modelos, quando os elementos de fora são muito pequenos, a energia livre se comporta de acordo com uma competição entre os termos da diagonal, sendo cada um deles dominante em uma das quase-fases.

Antes de prosseguir, vale fazermos uma ressalva sobre ponto crítico em modelos unidimensionais. O modelo de Ising unidimensional, como veremos, apresenta, em $(T, H)=(0,0)$, 
um ponto que, por um lado é dito ser crítico por exibir divergências na susceptibilidade e no comprimento de correlação, quando nos aproximamos de $T=0$, a campo nulo. Por outro lado, também apresenta uma descontinuidade na magnetização quando tomamos o limite para temperatura nula, a depender do sinal do campo. Ou seja, é um ponto que apresenta, simultaneamente, características de transição de primeira e segunda ordens. Adotaremos a noção de criticalidade como trazida em [4], como divergências nas segundas derivadas da energia livre, mas sublinhamos a existência dessa característica anômala em modelos unidimensionais. Não encontramos, na literatura, algum texto que desenvolva essa questão, mas acreditamos que seja algo que vale ser investigado.

Em [21], Rojas conjectura um critério para avaliar a possível ocorrência das pseudo-transições a partir da entropia residual $\left(S_{R}\right)$ do sistema, quer dizer, a entropia à temperatura nula. A proposta é que se a entropia residual no ponto crítico, dita entropia residual crítica $\left(S_{c}\right)$ é contínua com a entropia residual de ao menos uma das fases adjacentes, então é possível observar uma pseudo-transição próxima a esse ponto. A figura (1.4) é similar à apresentada no artigo e ilustra os possíveis cenários dessa afirmação. Os gráficos na parte de cima sugerem a existência da pseudo-transição, pois indicam a continuidade das entropias residuais. A justificativa é que a continuidade, e portanto a diferenciabilidade, do ponto crítico com uma das fases, faz com que a entropia aumente suavemente junto com a temperatura, levando consigo os vestígios da transição à temperatura nula e a informação sobre as fases adjacentes no estado fundamental.

Como apresentaremos, nos deparamos com um modelo que foge dos cenários propostos pela conjectura. Talvez um dos problemas esteja relacionado à definição de entropia residual crítica, que não está muito bem estabelecida, pois já que é possível obter essa quantidade a partir de vários caminhos distintos, não encontramos na literatura um critério formal para avaliar qual das entropias no estado fundamental é, com efeito, a entropia residual. Na dissertação, consideramos a entropia residual como sendo àquela tomada sobre a pseudo-interface, figura (4.7).

No capítulo 2, trataremos brevemente sobre o conceito biológico de lipídios e membranas biológicas, além de uma apresentação de alguns modelos que descrevem membranas artificiais. No capítulo 3, vamos comparar a resolução do gás ideal no contínuo e na rede, de modo a justi- 

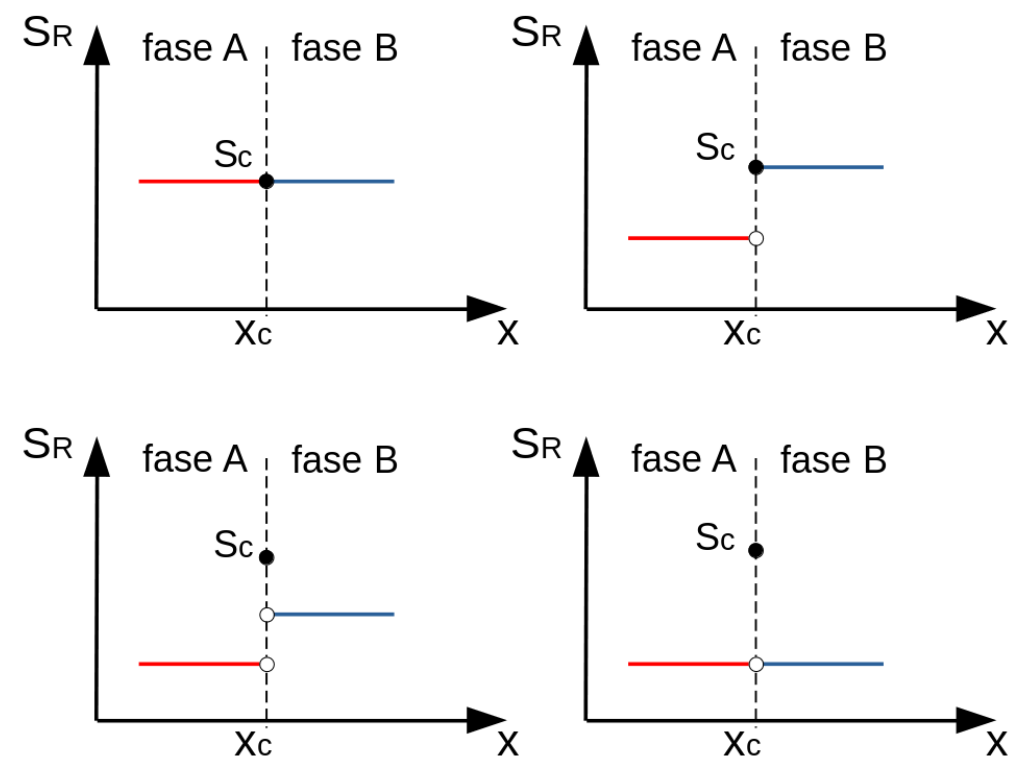

Figura 1.4: Ilustração esquemática da entropia residual do sistema no ponto crítico e nas fases adjacentes. O parâmetro $x$ é alguma variável do sistema, como campo externo ou potencial químico. A entropia residual crítica $S_{c}$ é a entropia residual no ponto crítico. Os dois casos na parte superior indicam a existência de pseudo-transição enquanto os da parte inferior indicam a inexistência.

ficar o uso deste último nesse trabalho, inserir interação entre as partículas do sistema e mostrar a propriedade de equivalência entre modelos com estruturas matemáticas semelhantes e apresentar a aproximação de campo médio para o modelo de Ising e o modelo de Ising degenerado. No capítulo 4 vamos resolver o modelo de Ising e o fluido de rede, unidimensionais, com e sem degenerescência, a partir do método da matriz de transferência. No capítulo 5 resolveremos o fluido de rede de Doniach unidimensional. Por fim, no capítulo 6 apresentaremos algumas conclusões e comentários finais. 


\section{Capítulo 2}

\section{Biomembranas e modelos}

\subsection{Lipídios, membranas e modelos experimentais}

Os lipídios são os blocos fundamentais com os quais são construídas as membranas biológicas, em especial as membranas plasmáticas, paredes que separam o meio interno do meio externo das células animais. Essas moléculas são ditas anfifílicas, ou anfipáticas, o que significa que possuem uma porção com afinidade com água, a cabeça polar, e uma porção hidrofóbica, as duas caudas apolares de ácido graxo. A classificação dos lipídios vai depender tanto da composição das cabeças quanto da composição e tamanho das caudas. Nas biomembranas, o principal tipo encontrado é o fosfolipídio, o qual possui o grupo fosfato na cabeça polar [1], como o que mostra a figura (2.1).
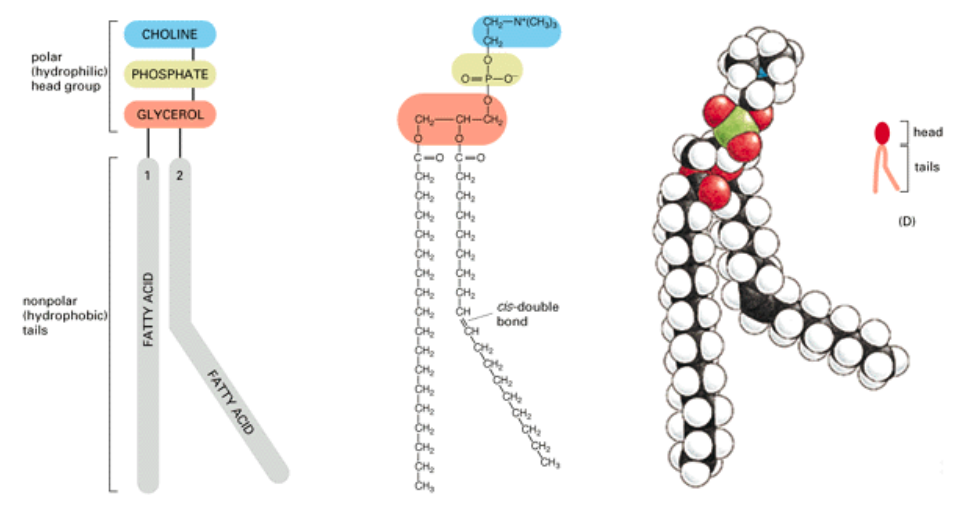

Figura 2.1: Fosfolipídios, molécula anfipática, composto por uma cabeça polar, feita de fosfato, colina e glicerol, e por duas caudas feitas de ácidos graxos. Retirado de http://cadernomedicina.blogspot.com/2015/01/o-fosfolipidio-o-muro-das-celulas.html, dia 21 de maio de 2020 às 08:54. 
As membranas plasmáticas, que mantém a coesão das células animais, têm uma composição essencialmente lipoproteica, feita principalmente de fosfolipídios, mas também de colesterol, glicolipídios, proteínas e carboidratos em menor quantidade, como mostra a figura (2.2). Esse sistema complexo é responsável por vários processos bioquímicos e físicos essenciais à vida, como a permeabilidade seletiva, regulando a composição no interior das células — tais como permitir a passagem de nutrientes importantes e a saída de produtos das organelas - , a comunicação intercelular, trocando informações com outras células do meio, o crescimento e a divisão celular por mitose e meiose [1].

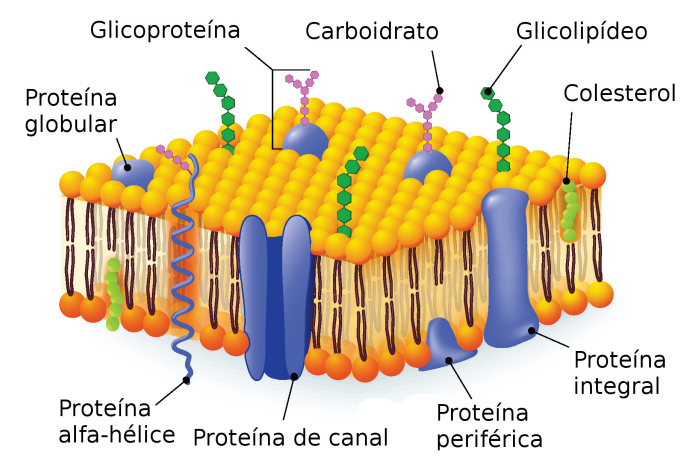

Figura 2.2: Membrana plasmática, com todos os demais componentes químicos complexos. Retirado de https://www.infoescola.com/citologia/membrana-plasmatica/, dia 19 de maio de 2020, às 21:26.

A propriedade anfipática obriga os lipídios em solução aquosa ou em tampão, a se agregarem em estruturas organizadas, já que suas caudas sempre procuram evitar o contato com a água. As arquiteturas formadas dependem de alguns fatores como a concentração e o local onde ocorre a agregação, mas as principais são as monocamadas na incerface água-ar, chamadas monocamadas de Langmuir, e as monocamadas e bicamadas no interior da solução, ditas micelas e lipossomos, respectivamente, como as mostradas na figura (2.3). A estabilidade dessas formações se deve a uma combinação das forças hidrofóbicas entre os lipídios e a água e entre uma interação de van de Waals entre os lipídios e lipídios. Eventualmente, também ocorrem interações eletrostáticas por conta de lipídios carregados.

Por conseguinte, as membranas devem ser, simultaneamente, estáveis, maleáveis e semipermeáveis. O estudo experimental das propriedades físicas de vesículas modelo — vesículas feitas artificialmente a partir de um único tipo de lipídio — têm dado pistas importantes sobre as suas funções biológicas [39].

Assim como outros sistemas físicos, quando há uma grande quantidade de componentes 


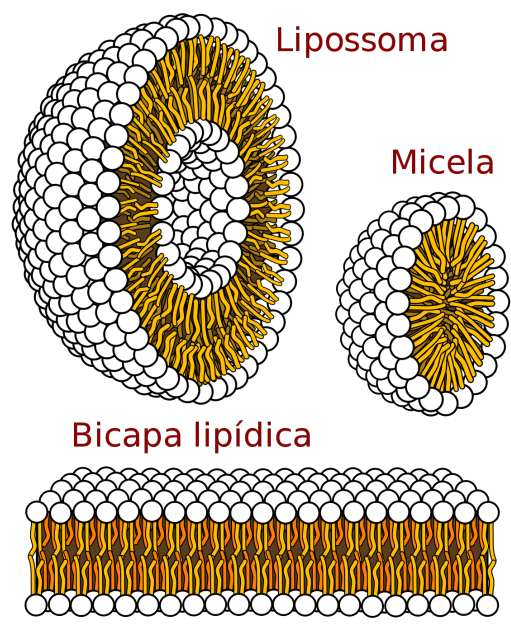

Figura 2.3: Em solução aquosa os lipídios se agregam em estruturas devido à natureza hidrofóbica das caudas. Algumas das principais são os lipossomos, as micelas e as bicamadas. Retirado de https://pt.wikipedia.org/wiki/Lipossoma, dia 19 de junho de 2021 às 11:21.

interagindo entre si, há a possibilidade da existência de propriedades emergentes, como fases termodinâmicas distintas, com mudanças abruptas nas características da estrutura, que só surgem devido a todas essas interações. Sob a variação de temperatura, concentração e pressão lateral no caso das monocamadas de Langmuir, é possível observar transições de fase em biomembranas.

A mais estudada, e a que trataremos aqui, é a transição ordem-desordem, quando as moléculas passam de um estado ordenado ou gel, para um estado desordenado ou fluido, sob o aumento da temperatura, figura (2.4). Experimentos mostram que nessa transição se observa um aumento da mobilidade dos lipídios bem como um aumento da área superficial das membranas, além de um pico estreito no calor específico na temperatura de transição, figura (2.5). Esses resultados indicam que a estrutura das membranas nessas fases contrastantes está relacionada ao estado das caudas dos lipídios, assumindo configurações ordenadas, all-trans, na fase gel e desordenadas na fase fluida. Com efeito, essa hipótese explica o aumento da área por cabeça polar além do aumento da mobilidade dos lipídios da membrana.

Para o estudo desses sistemas constituídos por uma quantidade grande de lipídios interagindo entre si pela ação hidrofóbica e o potencial de van der Waals, formando as estruturas e as fases termodinâmicas distintas, faremos uso da Mecânica Estatística para buscar uma descrição que traduza a configuração microscópica do sistema nas propriedades macroscópicas de 
Fase gel

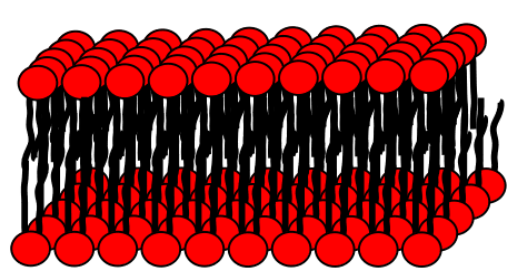

Fase fluido

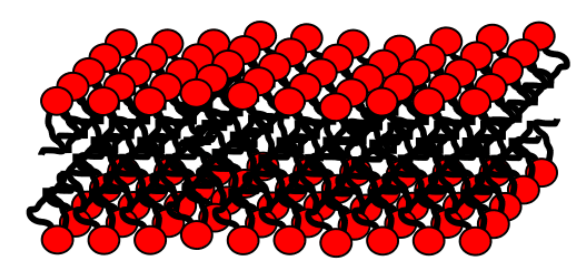

Figura 2.4: Ilustração esquemática da transição gel-fluido com a variação de temperatura. Na fase gel, as caudas dos lipídios lipídios da cadeia estão ordenadas, esticadas, enquanto na fase fluida os lipídios estão com as caudas apolares em um estado desordenado.
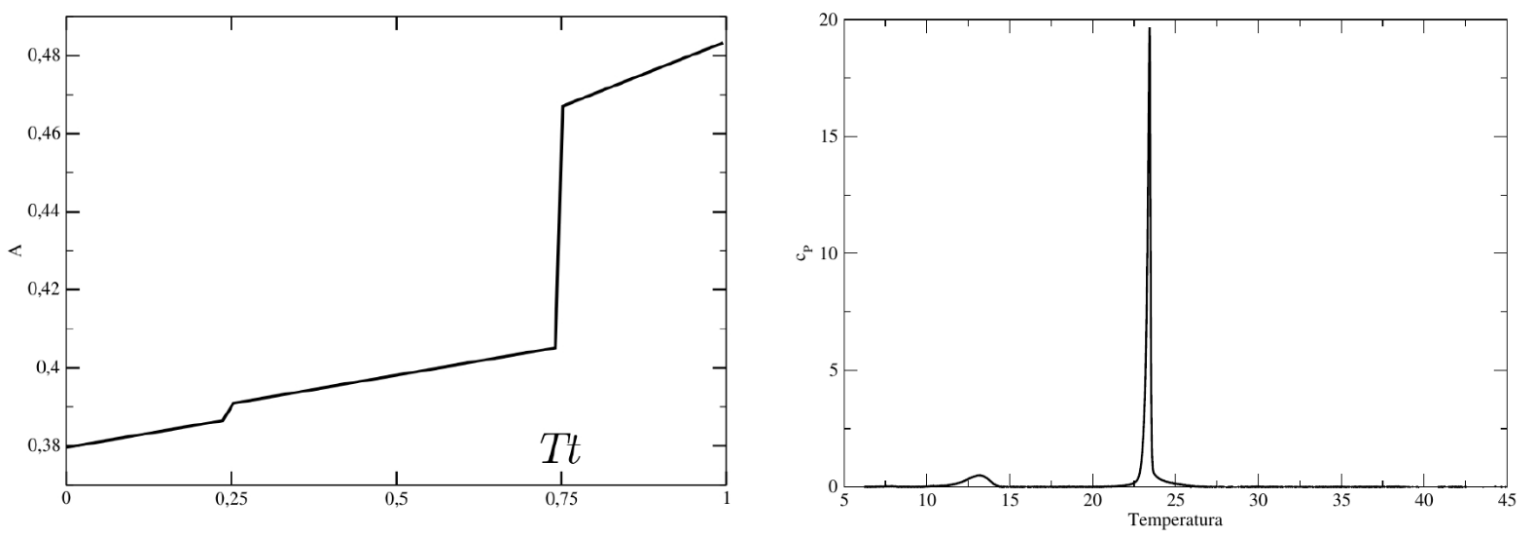

Figura 2.5: À esquerda, ilustração esquemática do aumento de área por cabeça polar em função da temperatura para bicamadas, onde $T_{t}$ é a temperatura de transição gel-fluido, retirado da referência [25]. À direita, calor específico a pressão constante em função da temperatura para solução aquosa de vesículas construídas com fosfatidil dimiristoil colina (DMPC), obtido no laboratório de biofísica do IFUSP, retirado da referência [36]. 
interesse, como densidade, entropia, calor específico, ordenamento médio, entre outros. Mais especificamente, usaremos modelos simplificados de rede baseados no modelo magnético de Ising para descrever as membranas. Entre os modelos conhecidos na literatura, o modelo de Doniach é o mais utilizado, vamos dedicar algumas linhas para explicar brevemente a sua construção.

\subsection{O modelo de Doniach - dois estados}

Doniach propõe descrever a membrana através de um modelo de dois estados. Cada sítio da rede bidimensional corresponde a um lipídio em um estado ordenado (relativo ao estado gel das caudas) ou desordenado (relativo ao estado fluido das caudas), como está representado na figura (1.1), onde este último possui uma alta degenerescência, de maneira a traduzir todas as possíveis dobras das caudas apolares. A energia de interação entre os lipídios é representada por uma interação atrativa de curto alcance, que tacitamente traduz os efeitos da característica hidrofóbica entre lipídios e água e a interação de van der Waals entre lipídios e lipídios. Podemos escrever a Hamiltoniana do sistema [8] como a soma sobre pares de primeiros vizinhos

$$
\mathcal{H}(\{\eta\})=-\epsilon_{o} \sum_{(i j)} \eta_{i} \eta_{j}-\epsilon_{d} \sum_{(i j)}\left(1-\eta_{i}\right)\left(1-\eta_{j}\right)-\epsilon_{o d} \sum_{(i j)}\left[\eta_{i}\left(1-\eta_{j}\right)+\left(1-\eta_{i}\right) \eta_{j}\right]
$$

onde $\epsilon_{o}, \epsilon_{d}$ e $\epsilon_{o d}$ são as energias de interação entre lipídios ordenado-ordenado, desordenadodesordenado e ordenado-desordenado, respectivamente, figura (2.6), sendo todas positivas desde que representam uma atração efetiva entre as moléculas, e $\eta_{i}=1$ para o estado ordenado e $\eta_{i}=0$ para o estado desordenado.
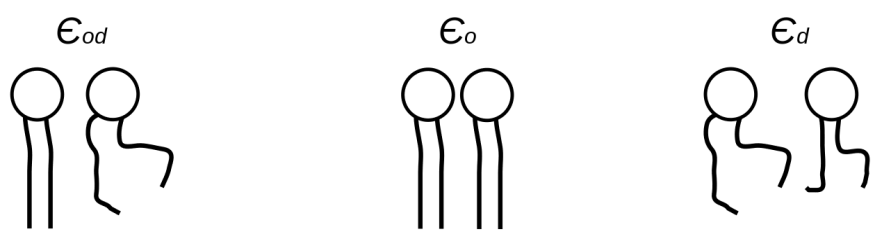

Figura 2.6: Esquema das energias de interação entre os lipídios de Doniach nos estados ordenado, com as caudas esticadas e desordenado, com as caudas flexionadas. 
A cauda com dobras ocupa um espaço maior do que a esticada, de modo que é atribuído a cada conformação uma área ocupada na superfície da membrana, sendo $a_{o}$ e $a_{d}$, para os estados ordenado e desordenado, respectivamente, com $a_{d}>a_{o}$. Assim, podemos definir uma área total ocupada pela monocamada

$$
A=\sum_{i}\left[a_{o} \eta_{i}+a_{d}\left(1-\eta_{i}\right)\right]
$$

Cumpre destacar que essa definição de área é feita $a d$ hoc, para que no ensemble das pressões, $(T, \Pi, N)$, seja possível considerar uma contribuição energética devido a uma pressão lateral $\Pi$, dada por $\Pi A$ - particularmente conveniente na descrição das monocamadas de Langmuir, por apresentarem transições de fase por conta dessa pressão.

Há também uma contribuição efetiva para a energia total do sistema de dois estados devido à degenerescência $\omega$, dada por

$$
E_{d e g}=\frac{\ln \omega}{2 \beta} \sum_{i}\left(\eta_{i}-1\right),
$$

onde $\beta=1 / k_{B} T$. Doniach obtém o diagrama de fases a partir do mapeamento do seu modelo com o modelo de Ising, figura (2.7). Isto é possível, pois a estrutura matemática de ambos é equivalente. Com efeito, o modelo de Doniach se trata de um modelo de Ising degenerado no contexto de biologia celular.

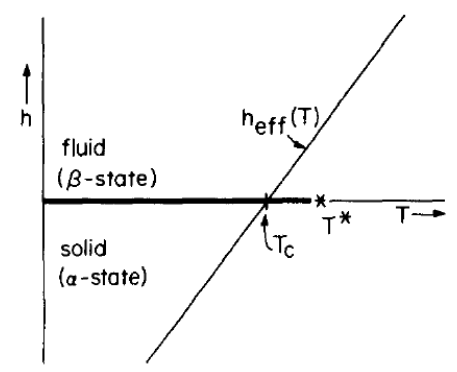

FIG. 1. Schematic phase diagram of a bilayer as a function of chain-entropy driving field $h$ and temperature $T$.

Figura 2.7: Esquema do diagrama de fases da bicamada, imagem retirada da referência [7] 


\subsection{O fluido de rede de Doniach - três estados}

Certos lipídios, como o DMPG (difosfatidildimiristoil glicerol), alteram a composição da cabeça polar perdendo um íon de sódio em solução, isto é, se tornam eletricamente carregados. Consequentemente, membranas formadas por essas moléculas apresentam comportamentos atípicos em baixa concentração de sal, se comparados àqueles observados para o DMPC (difosfatidildimiristoil colina) [26, 27], sufactante que apresenta uma estrutura muito similar ao do DMPG, exceto que a cabeça polar deste não dissocia em solução. Estudos baseados em modelos estatísticos sobre os efeitos da dissociação podem ser encontrados em [28, 29, 30].

Compreender o efeito das cargas sobre as propriedades termodinâmicas da membrana é o que motivou a modificação do modelo de Doniach por Henrique Guidi e Vera Henriques[8, 9], que buscaram redesenhar a descrição, de modo a tornar possível considerar interações eletrostáticas das cabeças ionizadas. Para isso, Guidi e Henriques propõe a construção de um modelo de três estados, com dois deles referentes aos estados ordenado e desordenado do modelo de Doniach usual e um terceiro representando um sítio vazio, de sorte que é possível considerar flutuações na densidade e, portanto, definir distâncias estatísticas entre os lipídios, permitindo a inserção da interação eletrostática.

Enquanto o modelo de Doniach coincide com o modelo de Ising degenerado de spin 1/2, o DLG é equivalente ao modelo de Ising degenerado de spin 1. Infelizmente este último não possui solução exata para mapearmos. Sua resolução na aproximação de campo médio foi feita por Guidi e Henriques em [9], e simulações de Monte Carlo por Guidi em [8]. Outras resoluções podem também ser encontradas em [31], feita por Vignoto, através de aproximação de campo médio em comparação com as monocamadas de Langmuir e também feitas por Oliveira e Tamashiro, por aproximação de pares, em [32, 33, 34].

Neste trabalho iremos aplicar o método da matriz de transferência na versão unidimensional do modelo para obter a solução exata. 


\section{Capítulo 3}

\section{Modelos estatísticos - soluções analíticas}

Neste capítulo vamos obter soluções analíticas para alguns modelos estatísticos com o objetivo de desenvolver algumas ideias que serão importantes no restante do trabalho. Em primeiro lugar vamos analisar o gás ideal em dois contextos, na rede e no contínuo, de maneira a confrontar alguns resultados. Em seguida definiremos modelos com interação atrativa de curto alcance e sua propriedade de equivalência, isto é, sua relação biunívoca através de um determinado mapeamento. Finalmente, resolveremos o modelo de Ising e o modelo de Ising degenerado através da aproximação de campo médio.

\subsection{Gás ideal}

\subsubsection{Na rede}

Considere uma rede quadrada de lado $L$, com $V=L^{2}$ sítios e contendo $N \leq V$ partículas, cada uma podendo ocupar um único sítio, como esquematizado na figura (3.1). Embora o gás ideal seja conhecido como um sistema em que as componentes não interagem entre si, no contexto de modelos na rede, o definiremos contendo uma interação repulsiva de curto alcance, que traduz a impossibilidade de duas partículas ocuparem o mesmo sítio na rede.

Representamos o estado do sítio através de $\eta_{i}=0,1$, onde o valor 0 significa que o sítio está vazio e o valor 1 , que está ocupado, com o índice $i=1,2, \ldots, V$ sendo a localização da partícula. Além disso, as variáveis são tais que 


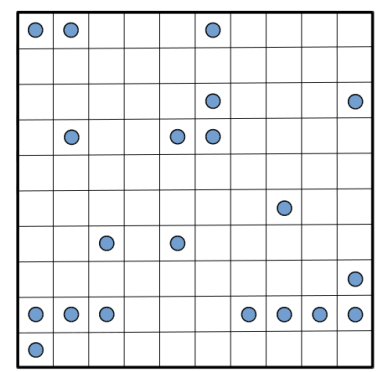

Figura 3.1: Representação esquemática de uma rede quadrada de lado $L$ com $N \leq V$ partículas. Cada célular pode conter, no máximo, uma partícula

$$
\sum_{i=1}^{V} \eta_{i}=N
$$

No ensemble microcanônico, o número total de possíveis configurações do sistema é

$$
\Omega(V, N)=\frac{V !}{N !(V-N) !}
$$

A conexão com a termodinâmica é feita a partir da entropia, definida como

$$
S=-k_{B} \ln \Omega
$$

Substituíndo $\Omega$ nessa expressão e fazendo uso da aproximação de Stirling

$$
\ln N ! \approx N \ln N-N \quad \text { quando } \quad N \gg 1
$$

teremos, no limite termodinâmico, para $V, N \rightarrow \infty \operatorname{com} v=V / N=1 / \rho$ fixo, uma expressão para a entropia em função de $V$ e $N$

$$
S(V, N)=k_{B}[V \ln V-N \ln N-(V-N) \ln (V-N)]
$$

ou, ainda, da propriedade de extensividade, a entropia por partícula

$$
s(v)=\frac{S}{N}=k_{B}[v \ln v-(v-1) \ln (v-1)]
$$

Agora precisamos lembrar da relação entre a variação das variáveis termodinâmicas num processo infinitesimal reversível [35, 36, 37] 


$$
d U=T d S-p d V+\mu d N
$$

É conveniente inverter a relação (3.7) para trabalharmos na representação da entropia, $S=$ $S(U, V, N)$, que nos coloca sobre o ensemble microcanônico, segue que

$$
d S=\frac{1}{T} d U+\frac{p}{T} d V-\frac{\mu}{T} d N
$$

Diferenciando $S$ e identificando seus coeficientes com aqueles da expressão (3.8), obtemos as equações de estado

$$
\frac{1}{T}=\left(\frac{\partial S}{\partial U}\right)_{V, N}, \quad \frac{p}{T}=\left(\frac{\partial S}{\partial V}\right)_{U, N}, \quad \frac{\mu}{T}=-\left(\frac{\partial S}{\partial N}\right)_{U, V} .
$$

A partir destas, assim como da entropia, em (3.5) ou (3.6), as quantidade termodinâmicas de interesse são facilmente calculadas

A pressão é dada por

$$
p=-k_{B} T \ln (1-\rho) .
$$

Se expandirmos em uma série de Taylor, obtemos

$$
p \approx k_{B} T \rho+\frac{k_{B} T \rho^{2}}{2}
$$

Agora vamos lembrar que, para o gás ideal, temos $\rho \ll 1$, o que nos fornece a conhecida equação de estado do gás ideal

$$
p=k_{B} T \rho .
$$

Por último, o potencial químico

$$
\mu=k_{B} T \ln \rho
$$

Com efeito, quanto mais preenchida estiver a rede, mais interações repulsivas são criadas en- 
tre os pares de partículas, sendo necessário cada vez mais energia para adicioná-las ao sistema. Ora, mas isso é precisamente o que a equação (3.7) nos conta, o potencial químico representa a variação da energia interna ao inserir uma partícula no sistema.

\subsubsection{No contínuo}

A função de partição canônica clássica pode ser obtida por

$$
Z(T, V, N)=\frac{1}{h^{3 N}} \int d^{3} \vec{r}_{1} \ldots \int d^{3} \vec{r}_{N} \int d^{3} \vec{p}_{1} \ldots \int d^{3} \vec{p}_{N} e^{-\beta \mathcal{H}}
$$

onde $\overrightarrow{r_{i}}$ e $\overrightarrow{p_{i}}$ são a posição e o momento linear da i-ésima partícula, respectivamente, e $h$ é a constante de Planck. Diferente do que ocorre com o modelo da rede, o sistema no contínuo não possui uma interação repulsiva de curto alcance, as partículas são consideradas pontuais e não há impedimento nas sobreposições entre elas. Como as interações são traduzidas pela parcela da energia associada à energia potencial, nesse contexto a energia total é composta somente da parcela associada ao movimento: a energia cinética. Assim, a Hamiltoniana do gás ideal é dada por

$$
\mathcal{H}=\sum_{i=1}^{N} \frac{\vec{p}_{i}^{2}}{2 m}
$$

onde $m$ é a massa das partículas. O fato de o sistema ser composto por elementos que não dialogam entre si — veja que, enquanto na rede nós desconsideramos o movimento, aqui nós desconsideramos a interação —, torna a resolução da integral (3.14) um trabalho um pouco mais fácil, já que desacopla as exponenciais transformando a função de partição em um produto entre o volume $V^{N}$, dado pela parte configuracional, e um conjunto de integrais gaussianas. Ademais, para uma solução que leve em consideração a indistinguibilidade entre as partículas do sistema, um fator $N$ ! deve ser adicionado manualmente

$$
Z(T, V, N)=\frac{V^{N}}{N !}\left(\frac{2 \pi m k_{B} T}{h^{2}}\right)^{\frac{3 N}{2}}
$$

Para facilitar a notação, vamos definir 


$$
\Lambda(T):=\left(\frac{h^{2}}{2 \pi m k_{B} T}\right)^{\frac{1}{2}}
$$

conhecido como comprimento de onda térmico, de modo que a função de partição (3.16) assume a forma

$$
Z(T, V, N)=\frac{V^{N}}{N ! \Lambda^{3 N}(T)}
$$

Note que agora estamos sobre o ensemble canônico $(T, V, N)$, de sorte que precisamos utilizar equações de estado diferentes das relações (3.9). Em primeiro lugar, fazemos uma transformada de Legendre da energia interna, obtendo a energia livre de Helmholtz

$$
F=U-T S
$$

cuja diferencial, junto com a diferencial (3.7), nos fornece

$$
d F=-S d T-p d V+\mu d N
$$

Diferenciando a função $F(T, V, N)$ e comparando os termos, obtemos as equações de estado canônicas

$$
S=-\left(\frac{\partial F}{\partial T}\right)_{V, N}, \quad p=-\left(\frac{\partial F}{\partial V}\right)_{T, N}, \quad \mu=\left(\frac{\partial F}{\partial N}\right)_{T, V}
$$

A conexão com a termodinâmica é feita através da energia livre de Helmholtz — para o gás na rede, estávamos no ensemble microcanônico, portanto a conexão foi feita a partir da entropia -, dada por

$$
F=-k_{B} T \ln Z
$$

Substituindo (3.18) na expressão acima e evocando novamente a aproximação de Stirling (3.4), obtemos

$$
F(T, V, N)=-N k_{B} T[\ln V-\ln N+1-3 \ln \Lambda(T)]
$$


Assim, de (3.21), a pressão é facilmente obtida e note que é precisamente (3.12)

$$
p(T, \rho)=k_{B} T \rho .
$$

Finalmente, o potencial químico

$$
\mu(T, \rho)=k_{B} T \ln \rho+3 k_{B} T \ln \Lambda(T) .
$$

Observe que o primeiro termo da soma é também igual ao obtido na rede (3.13), porém obtivemos um termo adicional, relacionado ao movimento das partículas — não por acaso é dependente da temperatura.

Apresentamos duas abordagens para lidar com o problema de sistemas de muitos corpos com o objetivo de introduzir alguns dos conceitos que serão utilizados durante o trabalho que segue, e também para apresentar uma justificativa sobre a escolha em tratar modelos de rede. Como vimos, esse pano de fundo considera somente as interações entre as partículas do sistema e através delas conseguimos obter as quantidades de interesse. Entretanto, mesmo que o modelo no contínuo possibilite a obtenção de soluções a partir da energia cinética apenas, a adição de interação na função de partição (3.14) tornaria impossível uma resolução exata. O fato de modelos de rede possibilitarem a análise de sistemas interagentes, além de fornecer soluções exatas em muitos casos, a depender da complexidade do sistema, tornam essa abordagem essencial para a descrição de transições de fase, cujas interações desempenham papel central.

\subsection{Modelos interagentes na rede}

A interação entre as partículas é a chave para a compreensão das transições de fase, que é um fenômeno que emerge de sistemas com grandes quantidades de componentes agindo e interagindo de maneira complicada. Vamos introduzir nessa seção o mais famoso dos modelos de rede com interação de curto alcance: o modelo de Ising magnético, que servirá de base para os outros que veremos. Também apresentaremos o fluido de rede, matematicamente análogo ao primeiro.

A Hamiltoniana do modelo de Ising magnético é dada por 


$$
\mathcal{H}_{m}(\{\sigma\})=-J \sum_{(i, j)} \sigma_{i} \sigma_{j}
$$

onde $(i, j)$ representa uma soma sobre pares de primeiros vizinhos, $\sigma_{i}$ são as variáveis de spin, podendo assumir os valores -1 (spin down) e +1 (spin up) e $J$ é a energia de interação, positiva para o sistema ferromagnético, pois privilegia a formação de pares paralelos, e negativa para o sistema antiferromagnético, pois privilegia a formação de pares antiparalelos.

A Hamiltoniana do fluido de rede é dada por

$$
\mathcal{H}_{f}(\{\eta\})=-\epsilon \sum_{(i, j)} \eta_{i} \eta_{j}
$$

onde $\eta_{i}$ são as variáveis de ocupação, valendo 0 no caso de sítio vazio e 1 no caso de sítio preenchido, e $\epsilon>0$ é a energia de interação, que privilegia a agregação das partículas.

\subsubsection{Mapeamento do modelo de Ising no fluido de rede}

Uma característica importante de certos modelos físicos é a chamada universalidade, que fala sobre a equivalência entre certas classes de sistemas. Isto é, se dois modelos possuem estruturas matemáticas análogas, como as Hamiltonianas (3.26) e (3.27), é possível mapear os resultados de um no outro (ver cap.14 de [5]). Quer dizer, mesmo em contextos diferentes, a mesma arquitetura anui à construção de uma relação biunívoca entre as soluções desses sistemas.

Considere o seguinte mapeamento, que conecta o modelo de Ising ao fluido de rede

$$
\eta:=\frac{1+\sigma}{2}
$$

permitindo a relação

$$
\left\{\begin{array}{l}
\sigma=-1 \quad \rightarrow \quad \eta=0 \\
\sigma=+1 \quad \rightarrow \quad \eta=1
\end{array}\right.
$$

A magnetização, no modelo magnético, e o número de partículas, no fluido, são dadas, respectivamente, por 


$$
M=m_{0} \sum_{i} \sigma_{i}, \quad N=\sum_{i} \eta_{i}
$$

onde assumiremos $m_{0}=1$.

É possível obter uma importante relação entre a magnetização por partícula $m=M / V$ (veja que, no modelo de Ising, $V=N$ ) e a densidade $\rho=N / V$. Aplicando o mapeamento (3.28) em $N$

$$
\begin{gathered}
N=\sum_{i=1}^{V} \eta_{i}=\sum_{i=1}^{V} \frac{1+\sigma_{i}}{2}=\frac{V+M}{2}=V\left(\frac{1+m}{2}\right) \\
\rho=\frac{1+m}{2} .
\end{gathered}
$$

Considere as "Hamiltonianas"efetivas do modelo magnético $\mathcal{H}_{m}^{e f}$ e fluido $\mathcal{H}_{f}^{e f}$, obtidas de (3.26) e (3.27), respectivamente, através da adição de uma contribuição do campo externo para a primeira e do potencial químico para a segunda

$$
\mathcal{H}_{m}^{e f}=-J \sum_{(i, j)} \sigma_{i} \sigma_{j}-H \sum_{i} \sigma_{i}
$$

$\mathrm{e}$

$$
\mathcal{H}_{f}^{e f}=-\epsilon \sum_{(i, j)} \eta_{i} \eta_{j}-\mu \sum_{i} \eta_{i}
$$

Observe que $H$ e $\mu$ desempenham papéis análogos, ambos são termos de campo, quer dizer, estão associados aos componentes lineares das "Hamiltonianas"efetivas. Reescrevendo a equação (3.32) em termos das variáveis de spin, fazendo uso do mapeamento (3.28), obtemos

$$
\mathcal{H}_{f}^{e f}=-\frac{\epsilon}{4} \sum_{(i, j)} \sigma_{i} \sigma_{j}-\left(\frac{q \epsilon+2 \mu}{4}\right) \sum_{i} \sigma_{i},
$$

onde ignoramos a constante aditiva. Aqui, $q$ se refere à quantidade de primeiros vizinhos, por conseguinte, em uma rede quadrada, $q=4$, em uma cadeia unidimensional, $q=2$, e assim segue. Comparando as expressões (3.33) e (3.31), vemos, primeiramente, uma relação simples 
entre as energias de interação: $\epsilon=4 J$. Por outro lado, o que realmente nos interessa são os fatores associados aos termos lineares

$$
H=\frac{q \epsilon+2 \mu}{4}
$$

Aqui reside o triunfo do mapeamento entre os modelos da mesma classe de equivalência. Sabendo que o modelo de Ising possui uma solução exata para uma rede bidimensional quadrada [6], que fornece uma linha de transição em $H=0$, através da relação (3.34) concluímos que, no fluido de rede bidimensional quadrado, a linha de coexistência está sobre $\mu=-2 \epsilon$. Em outras palavras, para resolver o fluido de rede, ou outro modelo correspondente, não precisamos passar pelo tour de force matemático que Lars Onsager passou quando nos presenteou com a resolução do modelo de Ising, podemos apenas fazer o mapeamento da solução.

\subsection{Aproximação de campo médio}

Não são muitos os modelos termoestatísticos que possuem uma solução exata. Por esse motivo o uso de métodos aproximados no tratamento dos problemas é amplamente usado, e um dos mais importantes é a aproximação de campo médio. Utilizaremos a abordagem de Bragg-Williams [3], onde nós trocaremos as interações de curto alcance do modelo de Ising (3.26), dependente da posição relativa entre as partículas, por um potencial médio para o sistema todo, que depende apenas do número de elementos, com está ilustrado na figura (3.2).

Interações de primeiros vizinhos

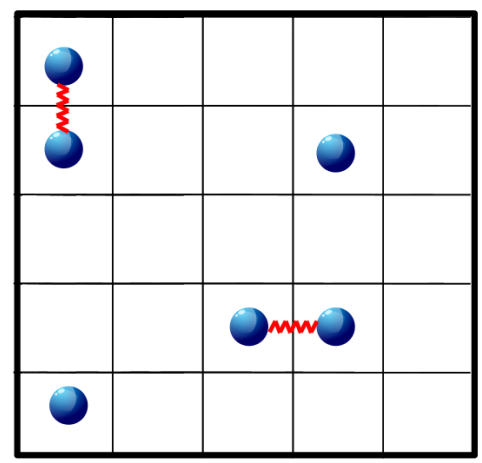

Interações de campo médio

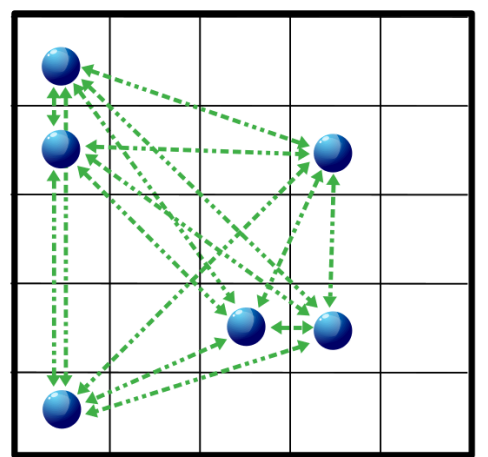

Figura 3.2: Representação esquemática de uma rede com interação de primeiros vizinhos à esquerda e uma rede com interação de campo médio à direita. 


\subsubsection{Modelo de Ising}

Considere um sistema magnético composto por $N$ spins, sendo $N_{+}$spins up e $N_{-}$spins down, de modo que podemos escrever o número de partículas $N$ e a magnetização $M$

$$
\left\{\begin{array}{l}
N=N_{+}+N_{-}, \\
M=N_{+}-N_{-},
\end{array}\right.
$$

cuja Hamiltoniana é a que já apresentamos, (3.26). Na aproximação de campo médio, vamos fazer as seguintes modificações

$$
\sigma_{i} \rightarrow\left\langle\sigma_{i}\right\rangle=m, \quad \sum_{(i, j)} \rightarrow \frac{q N}{2},
$$

em que a primeira se deve à aproximação de que o valor esperado de magnetização de todos os spins é tomado como uma constante e a segunda traduz uma independência das interações com relação à distância, sendo $q$ a quantidade de primeiros vizinhos. Estamos supondo que cada partícula interage com todas as outras, o fator 1/2 corrige uma contagem dobrada do número de interações. A Hamiltoniana (3.26) se torna

$$
\mathcal{H}_{c m}=-\frac{N q J}{2} m^{2}
$$

A função de partição pode ser escrita como

$$
Z_{c m}(T, m)=\Omega e^{-\beta \mathcal{H}_{c m}}
$$

onde $\Omega$ é a quantidade de maneiras possíveis de se arranjar $N_{+}$partículas com spin para cima e $N_{-}$com spin para baixo

$$
\Omega=\frac{N !}{N_{+} ! N_{-} !}
$$

Evocando a fórmula de Stirling (3.4) e usando a relação (3.35)

$$
\ln \Omega=N \ln 2-\frac{N}{2}[(1+m) \ln (1+m)+(1-m) \ln (1-m)]
$$


onde $m=M / N$. Assim, a energia livre por sítio será

$$
\begin{gathered}
f_{c m}(T, m)=\frac{1}{N} F_{c m}(T, m, N)=-\frac{1}{N} k_{B} T \ln Z_{c m}=-\frac{k_{B} T}{N}\left[\ln \Omega-\beta \mathcal{H}_{c m}\right] \\
f_{c m}(T, m)=-\frac{q J}{2} m^{2}+\frac{k_{B} T}{2}[(1+m) \ln (1+m)+(1-m) \ln (1-m)],
\end{gathered}
$$

onde o termo $k_{B} T \ln 2$ foi ignorado por ser uma constante que não fará diferença na análise. Vamos fazer uso da teoria de Landau para investigar o comportamento do sistema. Considere a função de Landau

$$
g_{L}(T, H ; m)=f_{c m}(T, m)-H m,
$$

ou, mais explicitamente, a partir de (3.40)

$$
g_{L}(T, H ; m)=-H m-\frac{q J}{2} m^{2}+\frac{k_{B} T}{2}[(1+m) \ln (1+m)+(1-m) \ln (1-m)]
$$

A extremização de (3.42) com relação à $m$ é a transformada de Legendre de $f_{c m}(T, m)$, trocando a variável $m$ por $H$, nos levando do ensemble canônico $(T, m)$ para o ensemble dos campos $(T, H)$ e definindo a energia livre

$$
g(T, H)=\min _{m}\left[g_{L}(T, H ; m)\right]
$$

ou seja, quando $m$ satisfaz

$$
\left(\frac{\partial g}{\partial m}\right)_{T, H}=0 \quad \Longrightarrow \quad-H-q J m+\frac{k_{B} T}{2} \ln \left(\frac{1+m}{1-m}\right)=0 .
$$

Desenvolvendo a expressão acima obtemos a equação de Curie-Weiss

$$
m=\tanh [\beta(H+q J m)] .
$$


Sob esta restrição, podemos descrever o nosso sistema no ensemble dos campos. Contudo, a equação (3.44) é transcendental, portanto não podemos obter uma solução analítica a partir dela. Felizmente, soluções numéricas são facilmente obtidas, como encontrar a intersecção entre as funções que representam os lados esquerdo e direito da igualdade.

Mas vamos dar um passo atrás e ir por outro caminho. Considere a função de Landau (3.42). Se lembrarmos que a magnetização do sistema magnético de Ising é seu parâmetro de ordem, significa que nas vizinhanças do ponto crítico ele deve se anular. Então vamos olhar para essa vizinhança expandindo (3.42) em uma série de MacLaurin [38] com relação à $m$

$$
g_{L}(T, H ; m)=g_{L}(T, H ; 0)+\left.\frac{\partial g_{L}}{\partial m}\right|_{m=0} m+\left.\frac{\partial^{2} g_{L}}{\partial m^{2}}\right|_{m=0} m^{2}+\left.\frac{\partial^{3} g_{L}}{\partial m^{3}}\right|_{m=0} m^{3}+\ldots
$$

Com um pouco de esforço algébrico, obtemos a função de Landau nas proximidades da criticalidade

$$
g_{L}(T, H ; m)=-H m+\frac{\left(k_{B} T-q J\right)}{2} m^{2}+\frac{k_{B} T}{12} m^{4}+\mathcal{O}\left(m^{6}\right) .
$$

O que devemos fazer agora é analisar como os mínimos dessa função se comportam — pois são eles que validam a energia livre (3.43) e, portanto, são os valores da magnetização do sistema —, especialmente com relação aos parâmetros $T$ e $H$.

Em primeiro lugar, se retirarmos o campo, (3.46) se torna par, destarte possui uma simetria com relação ao sinal da magnetização. Nessa condição, o que vai ditar o comportamento da função é o sinal do termo quadrático: quando for não negativo, a única possibilidade para haver um mínimo é $m=0$, já que a função é convexa. Por outro lado, quando este termo for negativo, teremos dois mínimos simétricos. Essas condições podem ser vistas na figura (3.3).

Vamos traduzir essas características para a física. Para altas temperaturas, $k_{B} T \geq q J$, há somente um mínimo, em $m=0$, o que é razoável, pois a alta temperatura torna os spins aleatórios. Em contrapartida, ao esfriar o sistema, $k_{B} T<q J$, a magnetização nula passa a ser um máximo, enquanto surgem dois novos mínimos simétricos em $m= \pm m_{0}$, que sinalizam o aparecimento de uma coexistência de fases sobre a linha $H=0$. Essa coexistência só ocorre 


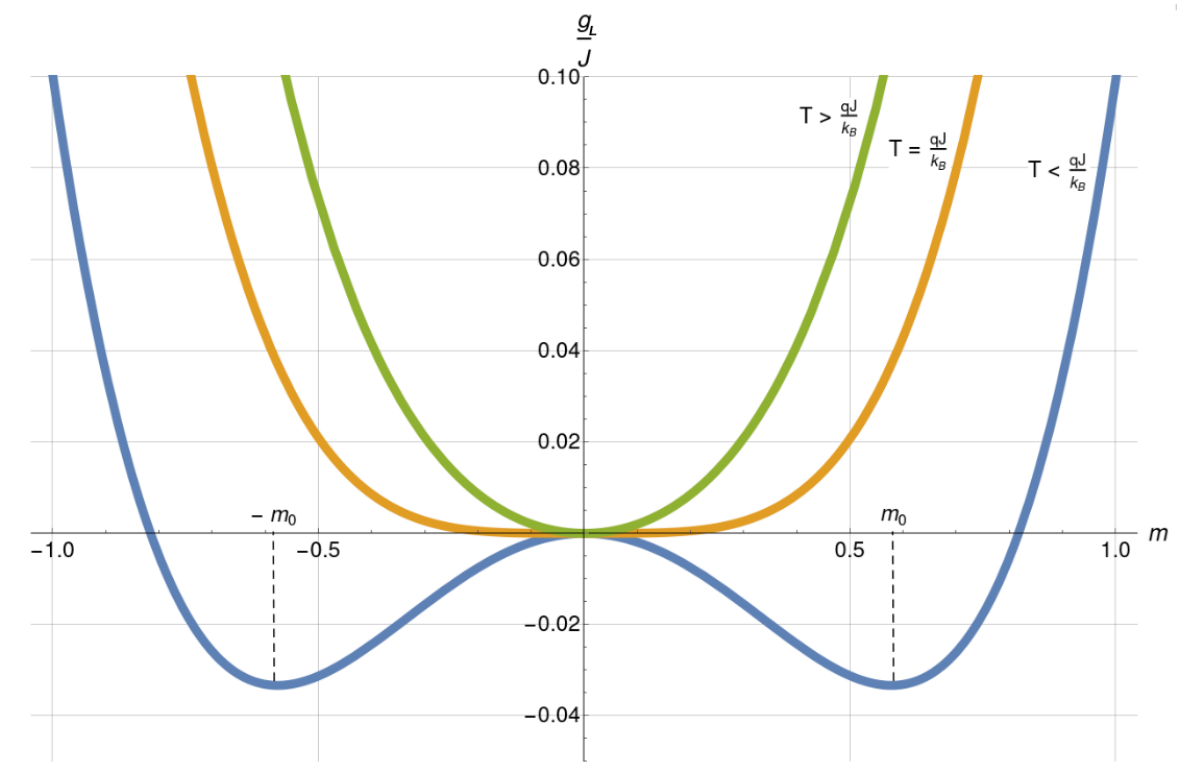

Figura 3.3: Funcão de Landau nas proximidades da temperatura crítica, para campo nulo, que nesse caso foi tomada como $k_{B} T_{c} / J=4$, para considerar uma rede bidimensional quadrada.

para temperaturas inferiores a $k_{B} T=q J$. Por esse motivo, definimos essa quantidade como a temperatura crítica $T_{c}:=q J / k_{B}$ do sistema. Cumpre também comentar que no caso em que temos campo não nulo, a função de Landau perde a simetria, possuindo um único mínimo global, que é a magnetização do sistema, como mostram os gráficos (c) e (d) da figura (3.5).

Vamos obter os valores desses mínimos simétricos $m_{0}$, para $H=0$. Derivando (3.46) e igualando a zero

$$
\begin{gathered}
\frac{\partial g_{L}(T, 0 ; m)}{\partial m}=0 \quad \Longrightarrow \quad\left(T-T_{c}\right) m+\frac{T}{3} m^{3}=0, \\
m=\left\{\begin{array}{lc}
0, & T \geq T_{c}, \\
\pm \sqrt{\frac{3\left(T_{c}-T\right)}{T}}, & T<T_{c} .
\end{array}\right.
\end{gathered}
$$

Note que $m$ não mostra sinais de descontinuidade sob a variação de $T$, o que caracteriza uma transição contínua ou de segunda ordem.

\subsubsection{Modelo de Ising degenerado}

Vamos fazer uma pequena modificação no modelo de Ising, de maneira a se adequar melhor ao nosso estudo de membranas. Considere que o estado $\sigma=-1$ seja $\omega$-degenerado, o 
que quer dizer que existem $\omega$ maneiras distintas de os spins se manifestarem com valor -1 , $\left(-1_{1},-1_{2}, \ldots,-1_{\omega}\right)$. A tradução matemática dessa característica é através da adição de um fator multiplicativo na função de partição (3.41)

$$
Z_{c m}=\Omega \omega^{N_{-}} e^{-\mathcal{H}_{c m}}
$$

onde $N_{-}$é definida pela relação (3.35). A energia livre será

$$
f_{c m}(T, m ; \omega)=-\frac{J q}{2} m^{2}+\frac{k_{B} T}{2}\left[(1+m) \ln (1+m)+(1-m) \ln \left(\frac{1-m}{\omega}\right)\right] .
$$

Vamos repetir o mesmo processo feito para o caso não degenerado. A função de Landau é dada por

$$
g_{L}(T, H ; \omega, m)=-H m-\frac{J q}{2} m^{2}+\frac{k_{B} T}{2}\left[(1+m) \ln (1+m)+(1-m) \ln \left(\frac{1-m}{\omega}\right)\right] .
$$

Novamente, a condição de extremização de $g_{L}$ nos fornece uma equação transcendental para a magnetização do sistema

$$
m=\frac{e^{\beta(q J m+H)}-\omega e^{-\beta(q J m+H)}}{e^{\beta(q J m+H)}+\omega e^{-\beta(q J m+H)}}
$$

Veja que para $\omega=1$, retornamos a equação de Curie-Weiss (3.44).

Contudo, vamos nos ater a olhar como o sistema se comporta nas vizinhanças do ponto crítico. Expandindo a função (3.50) em uma série de MacLaurin

$$
g_{L}(T, H ; m, \omega)=\left(\frac{k_{B} T \ln \omega}{2}-H\right) m+\frac{\left(k_{B} T-J q\right)}{2} m^{2}+\frac{k_{B} T}{12} m^{4}
$$

Agora o termo linear possui uma contribuição da degenerescência dos spins, produzindo um campo efetivo 


$$
H_{e f}:=\frac{k_{B} T \ln \omega}{2}-H
$$

De modo que a função pode ser reescrita com uma estrutura semelhante a da expressão (3.46)

$$
g_{L}(T, H ; m, \omega)=H_{e f} m+\frac{\left(k_{B} T-J q\right)}{2} m^{2}+\frac{k_{B} T}{12} m^{4}+\mathcal{O}\left(m^{6}\right) .
$$

Para campo efetivo nulo, o sistema possui novamente uma simetria em relação ao sinal de $m$ e temos novamente as soluções (3.46), cujo ponto crítico é dado por $k_{B} T_{c}=J q$. Além disso, essa condição também implica em uma relação entre campo e temperatura que sugere uma linha de coexistência dependente da degenerescência, dada por

$$
H=\frac{k_{B} T \ln \omega}{2}
$$

A relação acima está expressa no diagrama de fases da figura (3.4). Veja que a temperatura crítica é sempre a mesma, independente da degenerescência. Na figura (3.5), apresentamos novamente a função de Landau para diferentes pontos do diagrama de fases. Em (b), temos o mesmo comportamento apresentado na figura (3.3), para $H / J=0$, com efeito, esta é a linha de coexistência para o caso não degenerado. Em (c) e (d) também mostramos pontos um pouco abaixo e um pouco acima da linha de transição descontínua para mostrar como a função de Landau perde a sua simetria, i.e., deixam de coexistir dois valores possíveis de magnetização, passando a ter um único mínimo global, que é a magnetização do sistema — positiva para valores acima e negativa para valores abaixo da coexistência.

Por fim, é também interessante investigar o caso em que o termo quadrático se anula, isto é, sobre a temperatura crítica, $k_{B} T_{c}=J q$, a linha tracejada na figura (3.5), temos

$$
g_{L}=H_{e f} m+\frac{q J}{12} m^{4}
$$

cujo valor extremo é dado por 


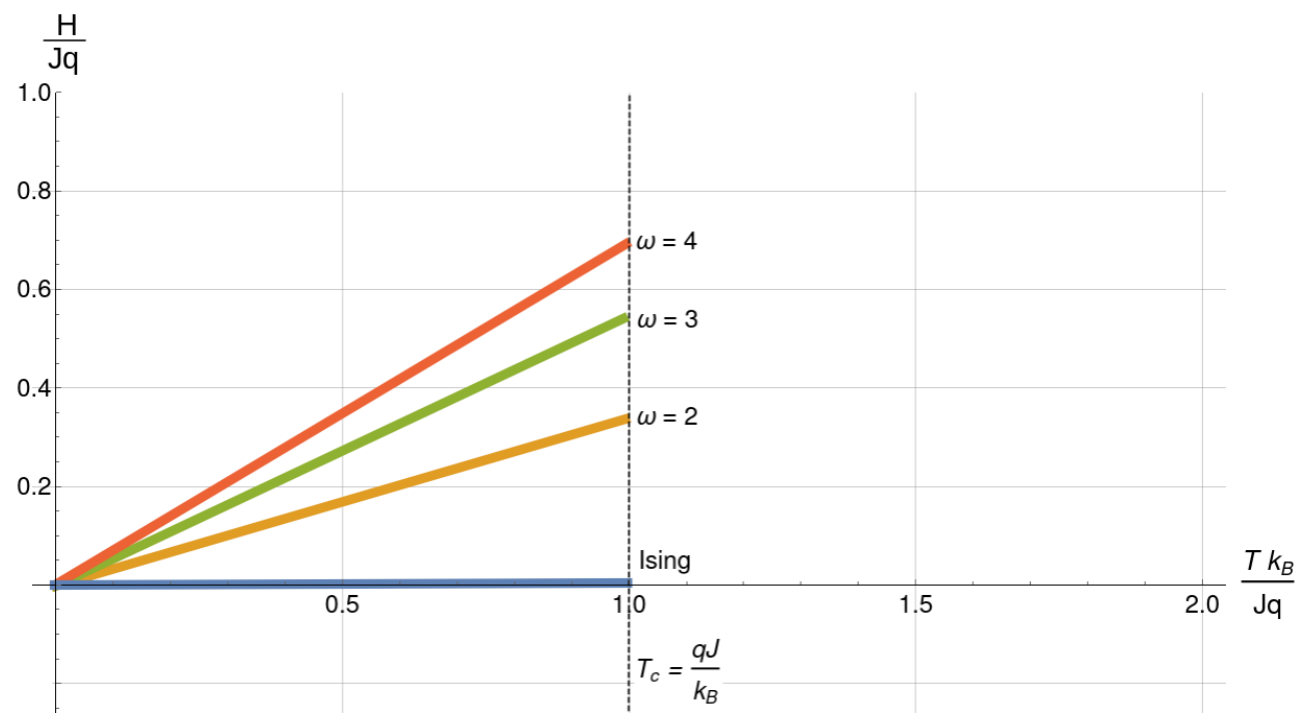

Figura 3.4: Diagrama de fase do modelo de Ising degenerado na aproximação de campo médio. As degenerescências mostradas são $\omega=1$ (Ising), 2, 3 e 4 . A linha tracejada se refere à temperatura crítica do modelo nessa aproximação.

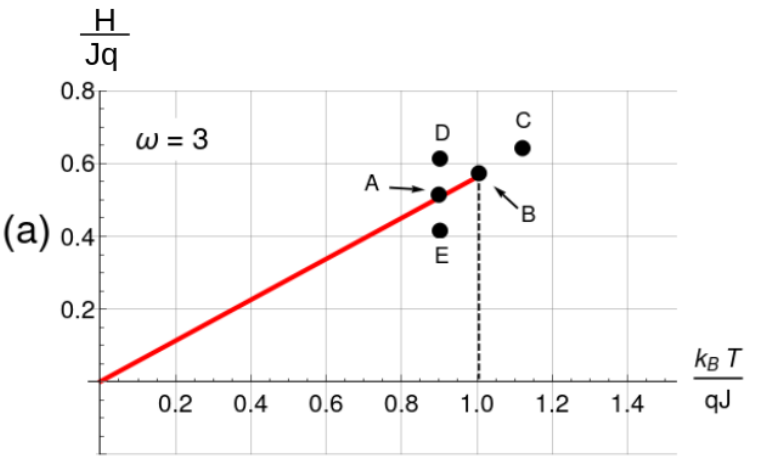

(b)

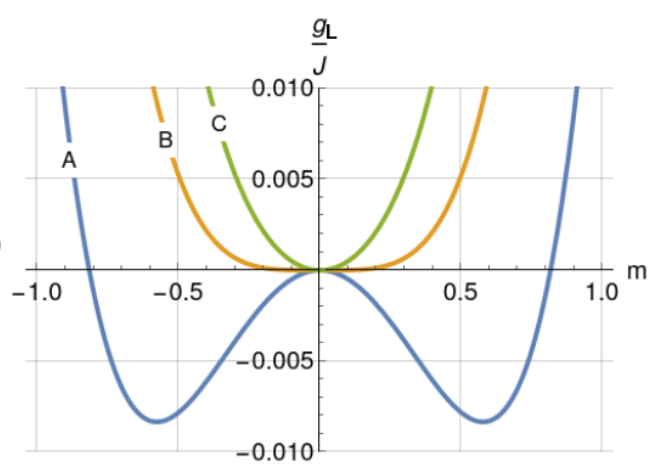

(c)

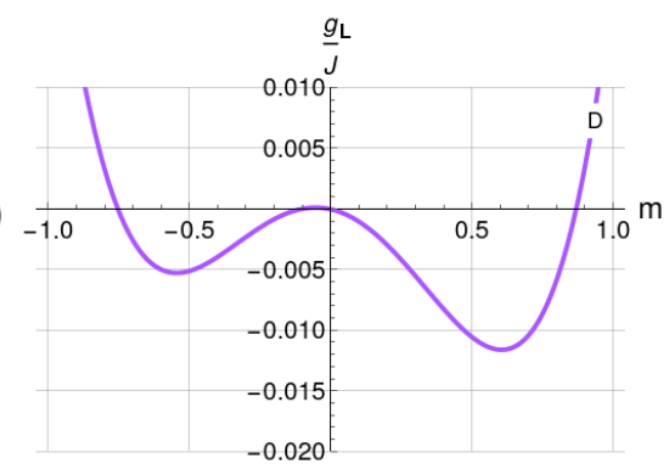

(d)

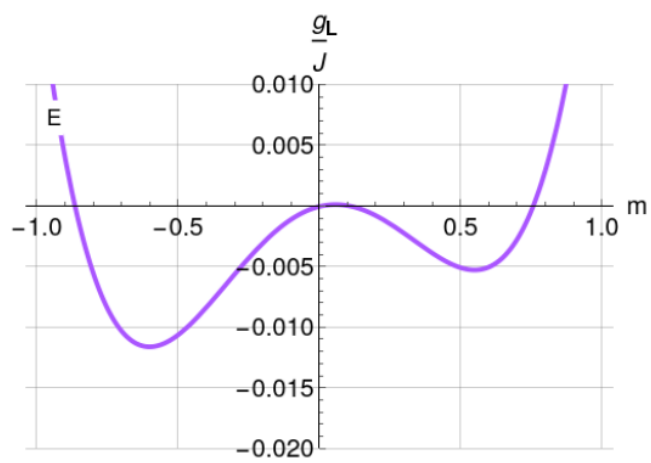

Figura 3.5: Modelo de Ising na aproximação de campo médio com degenerescência 3. Em (a), o diagrama de fases do modelo com os ponto $(T, H)$ fixados nos demais gráficos. Em (b), a função de Landau para os pontos $A$, e $B$ do diagrama de fases. Em (c) e (d), a função de Landau para, respectivamente, os pontos $D$ e $E$ no diagrama de fases. 


$$
\frac{\partial g}{\partial m}=0 \Longrightarrow m=-\sqrt[3]{3\left(\frac{\ln \omega}{2}-\frac{H}{J q}\right)}
$$

que se anula apenas quando vale (3.55), sendo positiva para $H>\frac{k_{B} T_{c} \ln \omega}{2}$ e negativo para $H<\frac{k_{B} T_{c} \ln \omega}{2}$, quer dizer, no ponto crítico já não temos a coexistência de fases, os spins já se tornaram arbitrários devido à temperatura.

Os resultados obtidos aqui para o modelo de Ising degenerado na aproximação do campo médio são interessantes, pois os confrontaremos com os que serão obtidos adiante para o mesmo modelo no caso unidimensional. 


\section{Capítulo 4}

\section{Modelos unidimensionais na rede}

Neste capítulo vamos analisar os modelos unidimensionais de dois estados, o ferromagneto de Ising e o fluido de rede, através do método da matriz de transferência - cuja demonstração pode ser vista no apêndice A —, além de resolver também estes sistemas com adição de degenerescência.

\subsection{Modelo de Ising unidimensional}

O modelo consiste em uma cadeia composta por $N$ partículas de spin - 1/2 que interagem entre si através de um potencial de curto alcance, expresso por uma interação de primeiros vizinhos. A Hamiltoniana (3.26) é acrescida de um termo linear associado ao campo magnético externo sobre os spins

$$
\mathcal{H}_{e f}(\{\sigma\})=-J \sum_{(i, j)} \sigma_{i} \sigma_{j}-H \sum_{i=1}^{N} \sigma_{i},
$$

onde $J>0$ é a energia de interação que favorece a organização paralela entre os vizinhos e que caracteriza um sistema ferromagnético - se tivéssemos $J<0$, a interação privilegiaria a configuração de vizinhos antiparalelos, o que caracteriza um sistema antiferromagnético.

No caso de uma dimensão, temos

$$
\mathcal{H}_{e f}(\{\sigma\})=-\sum_{i=1}^{N}\left(J \sigma_{i} \sigma_{i+1}+H \sigma_{i}\right) .
$$


É conveniente reescrever de forma mais simétrica

$$
\mathcal{H}_{e f}(\{\sigma\})=-\sum_{i=1}^{N}\left[J \sigma_{i} \sigma_{i+1}+\frac{H}{2}\left(\sigma_{i}+\sigma_{i+1}\right)\right] .
$$

A função de partição do sistema é dada por

$$
Z(T, H, N)=\sum_{\{\sigma\}} e^{-\beta \mathcal{H}_{e f}(\{\sigma\})}=\sum_{\{\sigma\}} \exp \left\{\sum_{i=1}^{N}\left[\beta J \sigma_{i} \sigma_{i+1}+\frac{\beta H}{2}\left(\sigma_{i}+\sigma_{i+1}\right)\right]\right\}
$$

O expoente com a somatória pode ser trocado pela produtória da exponencial

$$
Z(T, H, N)=\sum_{\{\sigma\}} \prod_{i=1}^{N} \exp \left[\beta J \sigma_{i} \sigma_{i+1}+\frac{\beta H}{2}\left(\sigma_{i}+\sigma_{i+1}\right)\right] .
$$

Em seguida, vamos definir o elemento de matriz

$$
T\left(\sigma_{i}, \sigma_{i+1}\right):=\exp \left[\beta J \sigma_{i} \sigma_{i+1}+\frac{\beta H}{2}\left(\sigma_{i}+\sigma_{i+1}\right)\right]
$$

que serão as entradas da matriz de transferência $\mathbb{T}$

$$
\mathbb{T}=\left[\begin{array}{cc}
T(-1,-1) & T(-1,1) \\
T(1,-1) & T(1,1)
\end{array}\right]=\left[\begin{array}{cc}
e^{\beta(J-H)} & e^{-\beta J} \\
e^{-\beta J} & e^{\beta(J+H)}
\end{array}\right],
$$

de tal sorte que (4.4) pode ser escrito de forma mais compacta

$$
Z(T, H, N)=\sum_{\{\sigma\}} \prod_{i=1}^{N} T\left(\sigma_{i}, \sigma_{i+1}\right) .
$$

Quando adotamos condições de contorno periódicas, i.e., $\sigma_{N+1}=\sigma_{1}$, podemos entender (4.7) como o traço da matriz $\mathbb{T}^{N}$ — apresentamos uma breve demonstração dessa afirmativa no apêndice A. Ademais, para uma matriz diagonalizável, os elementos da diagonal principal, na sua forma diagonal, são precisamente seus autovalores [39], segue que

$$
Z(T, H, N)=\lambda_{+}^{N}+\lambda_{-}^{N}
$$


onde $\lambda_{+}$e $\lambda_{-}$são os autovalores de $\mathbb{T}$. Essas quantidades podem ser obtidas através do cálculo das raízes do polinômio característico de $\mathbb{T}$, ou seja, resolvendo a equação

$$
\operatorname{det}(\mathbb{T}-\lambda \mathbb{I})=0
$$

que, nesse modelo, é dada pela equação do segundo grau em $\lambda$

$$
\lambda^{2}-2 e^{\beta J} \cosh (\beta H) \lambda+2 \sinh (2 \beta J)=0,
$$

cujas soluções são

$$
\lambda_{ \pm}=e^{\beta J}\left[\cosh (\beta H) \pm \sqrt{\sinh ^{2}(\beta H)+e^{-4 \beta J}}\right] .
$$

É fácil ver que $\lambda_{+}>\lambda_{-}$, dessa forma, no limite termodinâmico $N \rightarrow \infty$, vale

$$
Z(T, H, N)=\lambda_{+}^{N}+\lambda_{-}^{N}=\lambda_{+}^{N}\left[1+\left(\frac{\lambda_{-}}{\lambda_{+}}\right)^{N}\right] \longrightarrow \lambda_{+}^{N} .
$$

Finalmente, a função de partição do sistema é

$$
Z(T, H, N)=\left\{e^{\beta J}\left[\cosh (\beta H)+\sqrt{\left.\sinh ^{2}(\beta H)+e^{-4 \beta J}\right)}\right]\right\}^{N} .
$$

Agora devemos dar um pouco de atenção para a estrutura matemática sobre a qual estamos construindo o modelo. Nesse momento estamos no ensemble dos campos, $(T, H, N)$. Para obter as equações de estado, vamos tomar a transformada de Legendre (3.43)

$$
G=F-H M
$$

que nos permite trocar a energia livre de Helmholtz $F(T, M, N)$ pela energia livre com as variáveis apropriadas, $G(T, H, N)$. Diferenciando a transformada (4.13) e usando a diferencial (3.20) - levando em conta que, nesta última, haverá um termo adicional $+H d M$ e que podemos ignorar termo $-p d V-$, obtemos

$$
d G=-S d T+\mu d N-M d H
$$


cujas correspondentes equações de estado são

$$
S=-\left(\frac{\partial G}{\partial T}\right)_{N, H}, \quad \mu=\left(\frac{\partial G}{\partial N}\right)_{T, H}, \quad M=-\left(\frac{\partial G}{\partial H}\right)_{T, N},
$$

e a conexão com a termodinâmica é feita através de

$$
G(T, H, N)=-k_{B} T \ln Z(T, H, N) \text {. }
$$

Vamos escrever algumas quantidades termodinâmicas de interesse tornando explicita a relação com o maior autovalor (4.11).

A energia livre por partícula é

$$
g(T, H)=-k_{B} T \ln \lambda_{+}
$$

A entropia por partícula

$$
s(T, H)=k_{B} \ln \lambda_{+}+\frac{k_{B} T}{\lambda_{+}}\left(\frac{\partial \lambda_{+}}{\partial T}\right)_{H} .
$$

A magnetização por partícula

$$
m(T, H)=\frac{k_{B} T}{\lambda_{+}}\left(\frac{\partial \lambda_{+}}{\partial H}\right)_{T}
$$

A energia interna é obtida por

$$
U(T, H, N)=-\left[\frac{\partial \ln Z(T, H, N)}{\partial \beta}\right]_{H, N},
$$

de modo que a energia interna por partícula é

$$
u(T, H)=-\frac{1}{\lambda_{+}}\left(\frac{\partial \lambda_{+}}{\partial \beta}\right)_{H} .
$$

A susceptibilidade é definida por

$$
\chi(T, H)=\left[\frac{\partial m(T, H)}{\partial H}\right]_{T},
$$


assim

$$
\chi(T, H)=\frac{k_{B} T}{\lambda_{+}}\left(\frac{\partial^{2} \lambda_{+}}{\partial H^{2}}\right)_{T}-\beta m^{2}(T, H) .
$$

Por fim, o calor específico a campo constante

$$
c_{H}(T)=-T\left[\frac{\partial s(T, H)}{\partial T}\right]_{H},
$$

de maneira que

$$
c_{H}(T)=k_{B} \beta^{2}\left[\frac{1}{\lambda_{+}}\left(\frac{\partial^{2} \lambda_{+}}{\partial \beta^{2}}\right)_{H}-u^{2}(T, H)\right] .
$$

Explicitamente, a energia livre por partícula, a energia interna por partícula, a magnetização por partícula e a susceptibilidade são dadas por, respectivamente

$$
\begin{gathered}
g(T, H)=-J-k_{B} T \ln \left[\cosh (\beta H)+\sqrt{\left.\sinh ^{2}(\beta H)+e^{-4 \beta J}\right],}\right. \\
u(T, H)=-J-\frac{e^{\beta J}}{\lambda_{+}}\left[H \sinh (\beta H)+\frac{H \sinh (\beta H) \cosh (\beta H)-2 e^{-4 \beta J}}{\sqrt{\sinh ^{2}(\beta H)+e^{-4 \beta J}}}\right], \\
m(T, H)=\frac{\sinh (\beta H)}{\sqrt{\sinh ^{2}(\beta H)+e^{-4 \beta J}}}, \\
\chi(T, H)=\frac{\beta e^{-4 \beta J} \cosh (\beta H)}{\left[\sinh ^{2}(\beta H)+e^{-4 \beta J}\right]^{\frac{3}{2}}} .
\end{gathered}
$$

Na figura (4.1), apresentamos graficamente alguns dos resultados.

Primeiramente, no gráfico (b) vemos que, para temperaturas altas e $H \neq 0$, a magnetização vai assintóticamente a zero, o que é razoável, já que esperamos que a orientação dos spins se desfaça devido à energia térmica — quando $H=0$, a magnetização é nula para qualquer valor de temperatura. Em contrapartida, conforme o sistema esfria, a magnetização converge para $m=1,-1$ ou 0 , se o campo externo for positivo, negativo e nulo, respectivamente. Ainda 


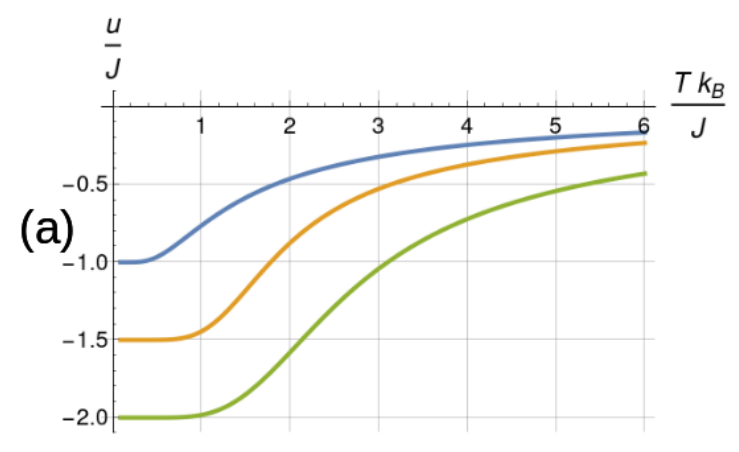

(b)

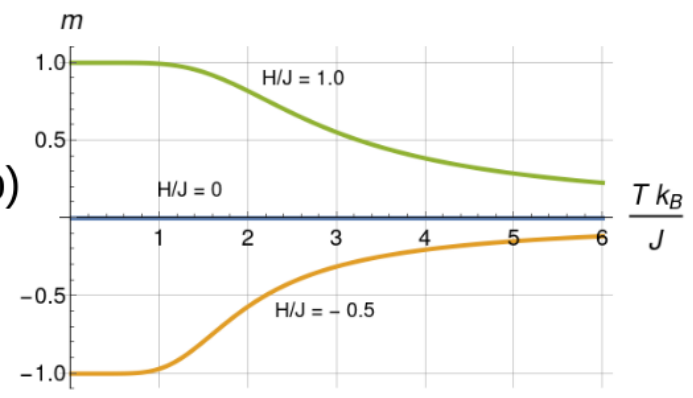

(c)
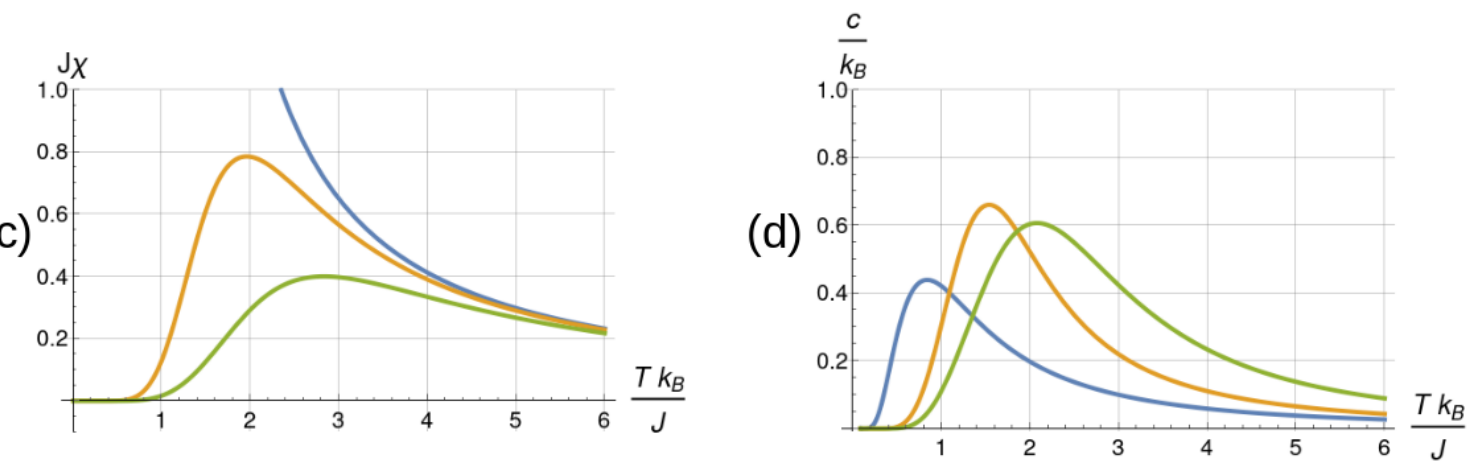

Figura 4.1: Em (a), a energia interna por partícula em função da temperatura, em (b) mostramos a magnetização por partícula em função da temperatura, em (c) a susceptibilidade em função da temperatura e em (d) o calor específico a campo constante em função da temperatura. Para todos os gráficos vale o padrão de cores azul, verde e amarelo para campos magnéticos de, respectivamente, $H / J=0,1 \mathrm{e}-0.5$

na curva da magnetização, é possível notar uma competição entre os efeitos da temperatura e do campo externo: quanto maior é o módulo do campo, mais lentamente é o decréscimo da magnetização, ou seja, maior é a resistência do sistema em perder a ordem.

Outro ponto para sobrelevar é a divergência na susceptibilidade mostrada no gráfico (c), quando diminuímos a temperatura na ausência de campo, sinalizando a existência de uma transição contínua no ponto $(T, H)=(0,0)$. Não obstante, o comportamento da magnetização no gráfico (b) também sugere uma transição descontínua. Com efeito, se tomarmos o limite da equação (4.28) para $H \rightarrow 0$ simultaneamente com $T \rightarrow 0$, o sinal da magnetização vai depender do caminho

$$
m(t \ll 1, H)= \begin{cases}+1 ; & H \rightarrow 0^{+} \\ 0 ; & H=0 \\ -1 ; & H \rightarrow 0^{-}\end{cases}
$$

onde $t:=\frac{k_{B} T}{J}$ é a temperatura reduzida. Por sua vez, o resultado (4.30) indica uma transição 
descontínua. Por conseguinte, a origem do diagrama $H-T$ do modelo de Ising unidimensional é, simultaneamente, um ponto de transição de primeira e segunda ordem. Não encontramos, na literatura, discussões aprofundadas sobre essa questão, o que é curioso pois é uma característica

que não se mostra nos modelos de dimensão maior. É possível que essa característica esteja relacionada ao fato de as linhas de pseudo-coexistência também possuírem características de transição de primeira e segunda ordens, apesar de não possuírem descontinuidade'.

\subsection{Modelo de Ising unidimensional degenerado}

No mesmo espírito da modificação que fizemos no modelo de Ising para tratá-lo pela aproximação de campo médio, vamos inserir na função de partição um fator que representa a degenerescência do estado -1, como na equação (3.48)

$$
Z=\sum_{\{\sigma\}} \omega^{N_{-1}} e^{-\beta\left(-J \sum_{i} \sigma_{i} \sigma_{1+i}-H \sum_{i} \sigma_{i}\right)}
$$

onde

$$
N_{-1}=\sum_{i}\left(\frac{1-\sigma_{i}}{2}\right)
$$

é a quantidade de spins -1 da cadeia. Desenvolvendo um pouco (4.31), obtemos

$$
Z=\sum_{\{\sigma\}} e^{N_{-1} \ln \omega} e^{-\beta\left(-J \sum_{i} \sigma_{i} \sigma_{1+i}-H \sum_{i} \sigma_{i}\right)}=\sum_{\{\sigma\}} e^{-\beta\left(-J \sum_{i} \sigma_{i} \sigma_{1+i}-H \sum_{i} \sigma_{i}-\frac{\ln \omega}{\beta} N_{-1}\right)} .
$$

Portanto, a "Hamiltoniana"efetiva, munida da contribuição do campo e da degenerescência, é

$$
\mathcal{H}_{e f}(\{\sigma\})=-J \sum_{i=1}^{N} \sigma_{i} \sigma_{i+1}-H \sum_{i=1}^{N} \sigma_{i}-\frac{\ln \omega}{2 \beta} \sum_{i=1}^{N}\left(1-\sigma_{i}\right) .
$$

Todas as etapas para a obtenção da função de partição que faremos a seguir são idênticas às feitas para o modelo de Ising. Reescrevendo (4.33) de forma simetrizada 


$$
\mathcal{H}_{e f}(\{\sigma\})=-\sum_{i=1}^{N}\left[J \sigma_{i} \sigma_{i+1}+\frac{H}{2}\left(\sigma_{i}+\sigma_{i+1}\right)+\frac{\ln \omega}{4 \beta}\left(2-\sigma_{i}-\sigma_{i+1}\right)\right]
$$

com correspondente matriz de transferência

$$
\mathbb{T}=\left[\begin{array}{cc}
T(-1,-1) & T(-1,1) \\
T(1,-1) & T(1,1)
\end{array}\right]=\left[\begin{array}{cc}
\omega e^{\beta(J-H)} & \sqrt{\omega} e^{-\beta J} \\
\sqrt{\omega} e^{-\beta J} & e^{\beta(J+H)}
\end{array}\right]
$$

e autovalores

$$
\lambda_{ \pm}=\frac{e^{\beta J}}{2}\left[e^{\beta H}+\omega e^{-\beta H} \pm \sqrt{\left(e^{\beta H}-\omega e^{-\beta H}\right)^{2}+4 \omega e^{-4 \beta J}}\right]
$$

Como antes, também é fácil ver que $\lambda_{+}>\lambda_{-}$, de sorte que a função de partição será, no limite termodinâmico, $Z=\lambda_{+}^{N}$. Explicitamente

$$
Z(T, H, N ; \omega)=\left\{\frac{e^{\beta J}}{2}\left[e^{\beta H}+\omega e^{-\beta H}+\sqrt{\left(e^{\beta H}-\omega e^{-\beta H}\right)^{2}+4 \omega e^{-4 \beta J}}\right]\right\}^{N}
$$

Fazendo uso novamente de (4.17), (4.18), (4.21) e (4.19), para o cálculo da energia livre por partícula, entropia por partícula, da energia interna por partícula e da magnetização por partícula, respectivamente, obtemos

$$
g(T, H ; \omega)=-J+k_{B} T \ln 2-k_{B} T \ln \left[e^{\beta H}+\omega e^{-\beta H}+\sqrt{\left(e^{\beta H}-\omega e^{-\beta H}\right)^{2}+4 \omega e^{-4 \beta J}}\right]
$$

$s(T, H ; \omega)=k_{B} \ln \lambda_{+}-\frac{1}{T}\left\{J+\frac{e^{\beta J}}{2 \lambda_{+}}\left[H\left(e^{\beta H}-\omega e^{-\beta H}\right)+\frac{H\left(e^{2 \beta H}-\omega^{2} e^{-2 \beta H}\right)-8 J \omega e^{-4 \beta J}}{\sqrt{\left(e^{\beta H}-\omega e^{-\beta H}\right)^{2}+4 \omega e^{-4 \beta J}}}\right]\right\}$ 


$$
\begin{gathered}
u(T, H ; \omega)=\frac{1}{\sqrt{\left(e^{\beta H}-\omega e^{-\beta H}\right)^{2}+4 \omega e^{-4 \beta J}}}\left\{\frac{4 J \omega\left(1+e^{-4 \beta J}\right)}{e^{\beta H}+\omega e^{-\beta H}+\sqrt{\left(e^{\beta H}-\omega e^{-\beta H}\right)^{2}+4 \omega e^{-4 \beta J}}}\right. \\
\left.-\left[(J+H) e^{\beta H}+(J-H) \omega e^{-\beta H}\right]\right\} \\
m(T, H ; \omega)=\frac{e^{\beta H}-\omega e^{-\beta H}}{\sqrt{\left(e^{\beta H}-\omega e^{-\beta H}\right)^{2}+4 \omega e^{-4 \beta J}}}
\end{gathered}
$$

É interessante obter as expressões acima para certos limites:

\section{Altas temperaturas}

$$
\begin{gathered}
g(t \gg 1, H ; \omega)=-J-k_{B} T \ln (\omega+1), \\
s(t \gg 1, H ; \omega)=k_{B} \ln (\omega+1), \\
u(t \gg 1, H ; \omega)=\frac{1}{1+\omega}\left\{\frac{4 \omega J}{1+\omega}-[(1+\omega) J+(1-\omega) H]\right\}, \\
m(t \gg 1, H ; \omega)=\frac{1-\omega}{1+\omega} .
\end{gathered}
$$

\section{Campo nulo}

$$
\begin{gathered}
g(T, 0 ; \omega)=-J+\frac{1}{\beta} \ln 2-\frac{1}{\beta} \ln \left[1+\omega+\sqrt{(1-\omega)^{2}+4 \omega e^{-4 \beta J}}\right] \\
s(T, 0 ; \omega)=k_{B} \ln \left[\frac{1+\omega+\sqrt{(1-\omega)^{2}+4 \omega e^{-4 \beta J}}}{2}\right] \\
-\frac{1}{T} \frac{1}{1+\omega+\sqrt{(1-\omega)^{2}+4 \omega e^{-4 \beta J}}} \frac{8 \omega J e^{-4 \beta J}}{\sqrt{(1-\omega)^{2}+4 \omega e^{-4 \beta J}}},
\end{gathered}
$$




$$
\begin{gathered}
u(T, 0 ; \omega)=\frac{4 \omega J}{(1+\omega)^{2}} \\
m(T, 0 ; \omega)=\frac{1-\omega}{1+\omega}
\end{gathered}
$$

\section{Baixas temperaturas}

$$
\begin{gathered}
g(t \ll 1, H ; \omega)= \begin{cases}-J-H, & H>\frac{k_{B} T}{2} \ln \omega, \\
-J, & H=\frac{k_{B} T}{2} \ln \omega, \\
-J+H, & H<\frac{k_{B} T}{2} \ln \omega .\end{cases} \\
s(t \ll 1, H ; \omega)= \begin{cases}\frac{k_{B} \ln \omega}{2}, & H=\frac{k_{B} T}{2} \ln \omega, \\
k_{B} \ln \omega, & H<\frac{k_{B} T}{2} \ln \omega .\end{cases} \\
u(t \ll 1, H ; \omega)=-J-|H|, \\
m(t \ll 1, H ; \omega)= \begin{cases}0, & H=\frac{k_{B} T}{2} \ln \omega, \\
+1, & H>\frac{k_{B} T}{2} \ln \omega, \\
-1, & H<\frac{k_{B} T}{2} \ln \omega .\end{cases}
\end{gathered}
$$

Observe que quase todas as expressões no limite para baixas temperaturas possuem duas sentenças, sob uma condição que é consequência da competição entre as exponenciais do termo $e^{\beta H} \pm \omega e^{-\beta H}$ presente em todas as funções de estado (4.38) - (4.41). Essa fronteira nos dá pistas sobre a possível existência de duas regiões que manifestam propriedades distintas, separadas pela linha

$$
H_{p}=\frac{k_{B} T}{2} \ln \omega
$$

Essa expressão já foi obtida anteriormente para o modelo de Ising degenerado na aproxima- 
ção de campo médio (3.55) e significa precisamente a linha de coexistência naquele caso. Aqui, por outro lado, entendemos essa separação como uma linha de pseudo-coexistência, uma fronteira entre duas quase-fases. Como mencionamos na introdução, essa terminologia foi utilizada pela primeira vez por Timonin [19] e se refere a fenômenos similares a transições de primeira e segunda ordem, simultaneamente. Não obstante, as descontinuidades ou divergências das funções de estado, características desses processos, não ocorrem - ao menos não em temperatura finita.

Essa linha pode ser vista no diagrama de quase-fases $H-T$ ao lado de uma diagrama de densidade de magnetização, na figura (4.7). É visível, pela magnetização do sistema, a existência de dois domínios distintos com uma separação tanto mais nítida quanto menor é a temperatura, se dissipando para altos valores de $T$.

\section{Matriz de transferência e pseudo-transições}

Outra forma de obter uma expressão para a linha de pseudo-coexistência é sugerida no trabalho de Rojas [20], que mostra que o fenômeno da pseudo-transição ocorre para modelos cujas matrizes de transferência possuem elementos fora da diagonal principal muito menores do que os desta. Quando o termo de fora da diagonal principal se torna muito pequeno, o que geralmente ocorre nas regiões de baixa temperatura, a energia livre se comporta segundo uma competição entre as entradas da diagonal principal, cada uma delas dominando em uma das quase-fases.

Vamos fazer uma breve avaliação das desigualdades mencionadas. As condições $T(-1,1)<$ $T(1,1)$ e $T(-1,1)<T(-1,-1)$, da matriz (4.35) implicam em, respectivamente

$$
H>\frac{k_{B} T}{2} \ln \omega-2 J \quad \text { e } \quad H<\frac{k_{B} T}{2} \ln \omega+2 J .
$$

Essas relações dizem que nas regiões entre a linha (4.54) e linhas paralelas deslocadas de uma quantidade $|2 J|$, o elemento $T(1,1)$ domina na parte superior e o $T(-1,-1)$, na parte inferior, como esquematizamos na figura (4.2).

Ademais, na matriz (4.35), o elemento $T(-1,-1)$ prevalece na quase-fase de magnetiza- 


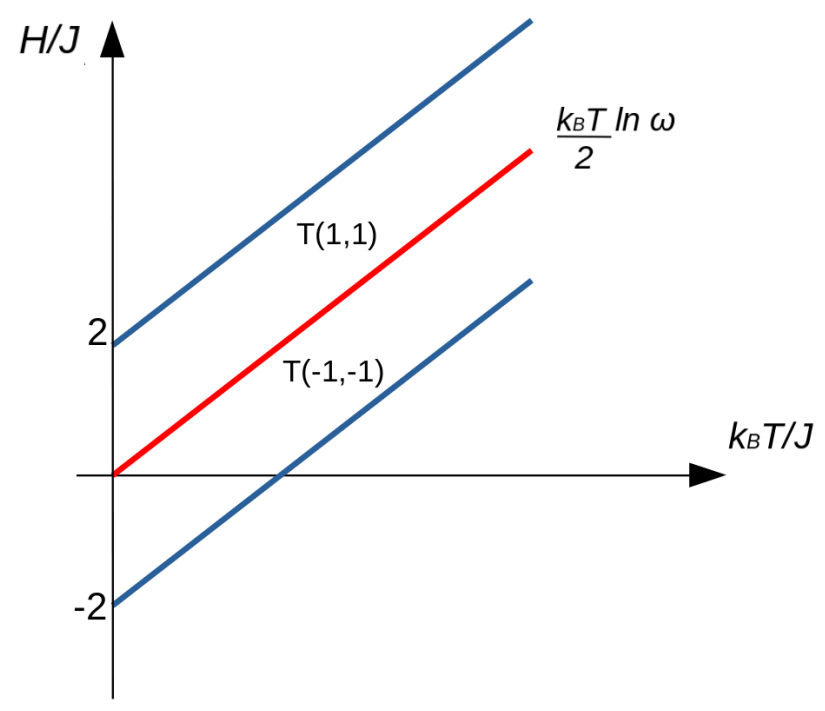

Figura 4.2: Ilustração esquemática do diagrama de quase-fases do modelo de Ising $\omega$-degenerado, a linha vermelha corresponde à pseudo-transição e as azuis são paralelas à esta deslocadas de uma quantidade \pm 2 verticalmente no gráfico.

ção -1 e $T(1,1)$, na de magnetização +1 , o que significa que a pseudo-transição se dá na coincidência desses termos

$$
T(-1,-1)=T(1,1) \Longrightarrow \omega e^{\beta(J-H)}=e^{\beta(J+H)}
$$

Não é difícil mostrar que esta é precisamente a relação (4.54). Também é possível ver que os termos $e^{-\beta J}$ sempre vão a zero quando resfriamos o sistema. Por outro lado, sempre que $-J \leq H \leq J$, ambos os termos da diagonal terão expoentes não-negativos, os colocando como termos dominantes na região de baixas temperaturas.

Convém fazermos uma última observação sobre o assunto. Recordando a discussão sobre transições de fase em modelos unidimensionais, comentamos que a possibilidade de transição está associada ao fato do sistema sair do alcance do teorema de Perron-Frobenius e, por conseguinte, implicar numa troca de maior autovalor da matriz de transferência. Ora, nas pseudotransições ocorre um mecanismo similar: há também uma troca de termo dominante, neste caso dado pelas entradas na diagonal principal da matriz de transferência, o que não implica em uma descontinuidade na energia livre, porém manifesta uma mudança abrupta nas características do sistema. 
Mostraremos alguns resultados obtidos nas figuras que seguem
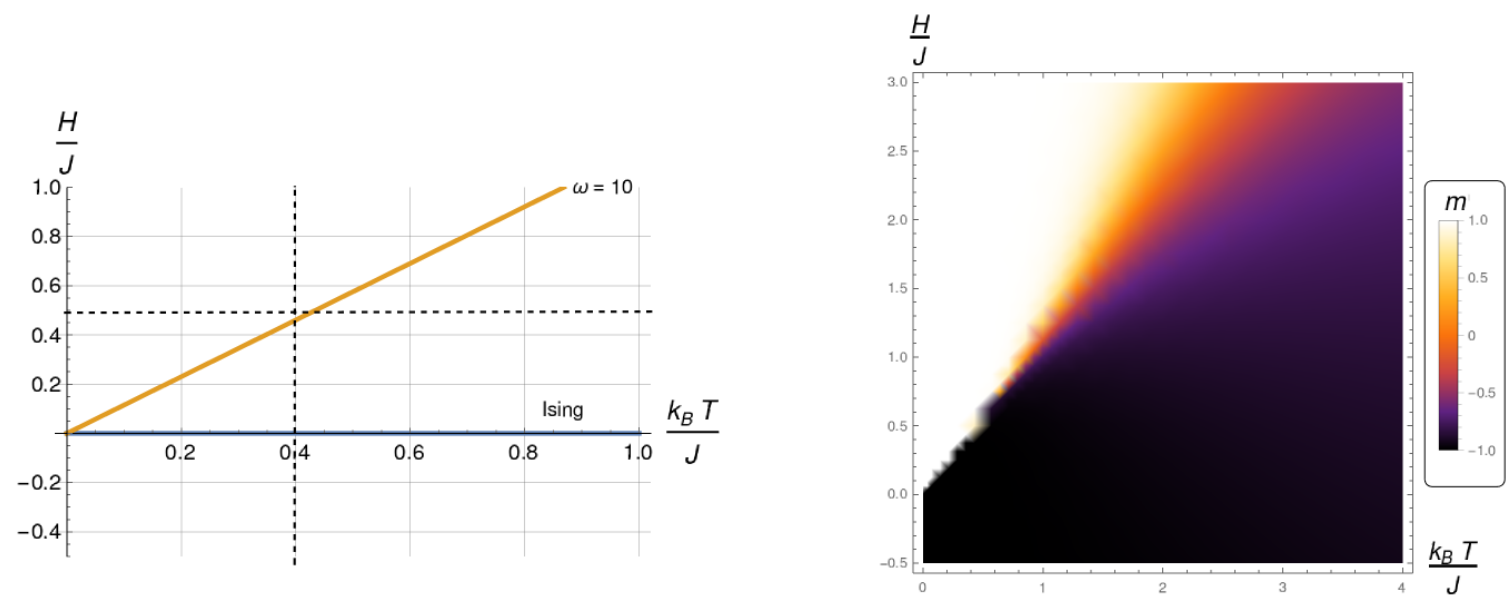

Figura 4.3: À esquerda o diagrama de quase-fases para o modelo de Ising e para o modelo de Ising com degenerescência $\omega=10$, as linhas tracejadas representam os cortes feitos para os gráficos das figuras (4.4) e (4.5), de campo magnético constante $H / J=0.5$ e temperatura constante $k_{B} T / J=0.4$, respectivamente. À direita, também para degenerescência 10, um diagrama de densidade para a magnetização cujos eixos são os mesmos do diagrama à esquerda. A região escura representa a magnetização -1 enquanto a região clara, a magnetização +1 .

Em primeiro lugar vamos colocar nossa atenção no comportamento do modelo com a inserção de degenerescência: a entropia e a magnetização em função de temperatura, mostradas na figura (4.4), tornam bastante visível a mudança brusca das características do sistema ao atravessar a linha de pseudo-transição da figura (4.3), ao caminharmos sobre a linha pontilhada horizontal. Veja que a magnetização, para altas temperaturas, é zero no modelo de Ising, porém é dada pela equação (4.49) no caso degenerado, que para $\omega=10$ vale $m=-0.81$. A maior quantidade de estados -1 faz com que o sistema não perca a magnetização, mesmo com o aumento de energia térmica. Também vemos que a degenerescência induz o aparecimento de um pico no calor específico e na susceptibilidade. Os dois efeitos mencionados são sinais de transições tanto de primeira ordem como de segunda, como esperado em quase-transições.

Com relação à figura (4.5), que corresponde ao caminho sobre a linha pontilhada vertical no diagrama (4.3), observamos o mesmo comportamento ao atravessar a pseudo-coexistência. O modelo de Ising $(\omega=1)$ também apresenta sinal de pseudo-transição ao atravessar a linha $H / J=0$, além disso, sua magnetização mostra uma mudança abrupta, idêntica à observada no modelo degenerado, porém localizada sobre a linha de campo nulo. O calor específico também apresenta um pico, apesar de pequeno em comparação ao sistema degenerado. A susceptibi- 

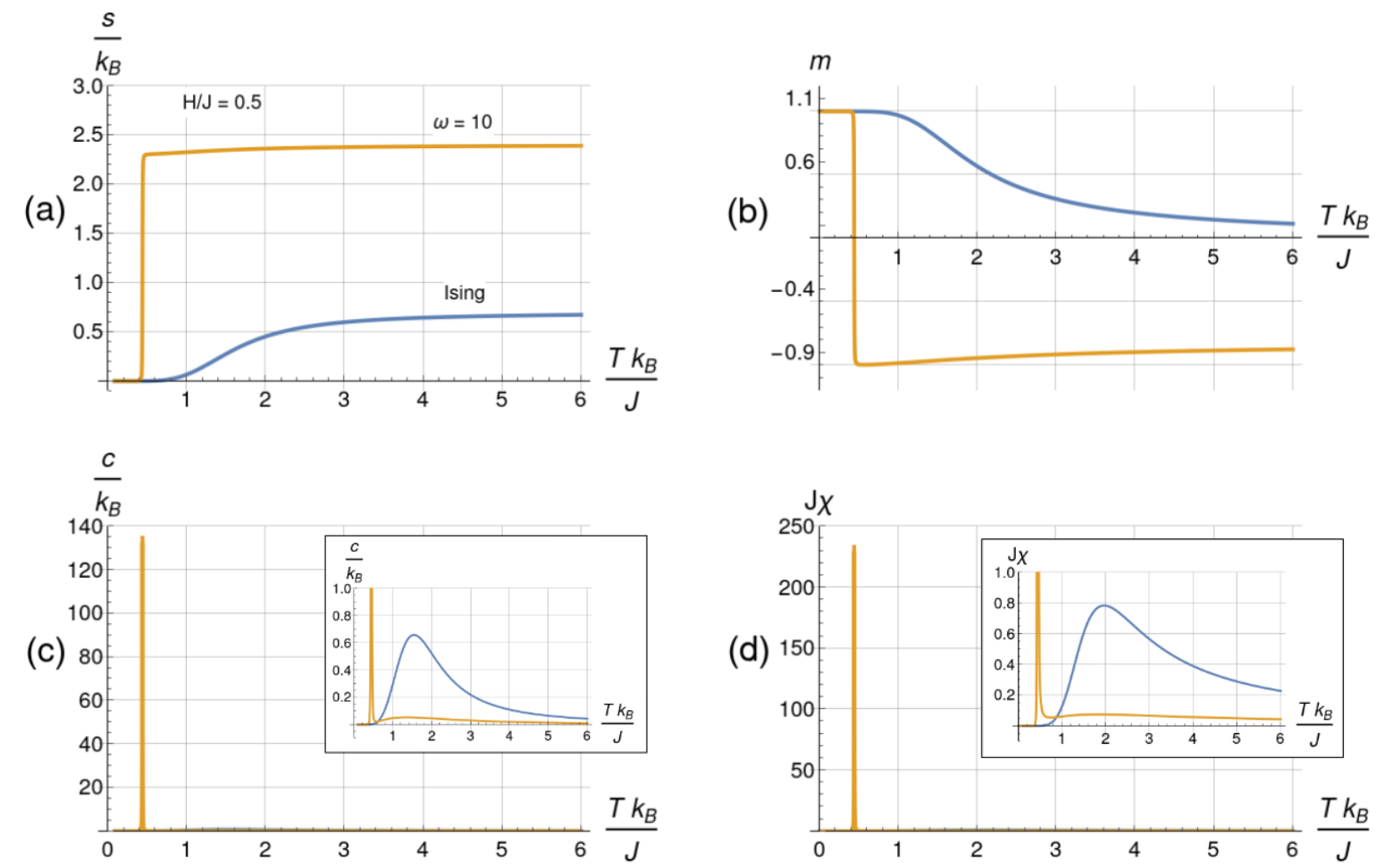

Figura 4.4: O gráfico (a) mostra a entropia por partícula em função da temperatura, em (b) a magnetização por partícula em função da temperatura, em (c) o calor específico a campo constante em função da temperatura e em (d) a susceptibilidade em função da temperatura. Em todas as figuras a curva azul representa o modelo de Ising enquanto a curva amarela representa o modelo de Ising com degenerescência $\omega=10$, ambos os sistemas imersos num campo magnético $H / J=0.5$. 
(a)

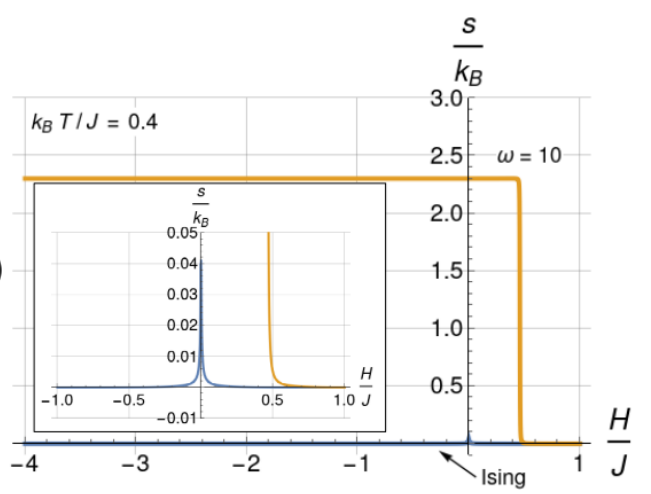

(c)

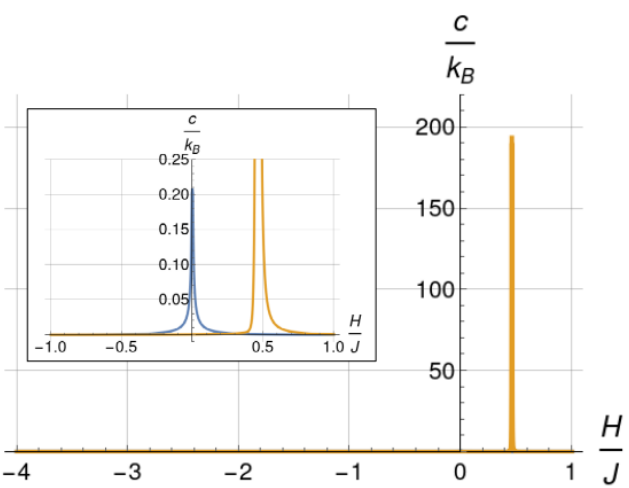

(b)

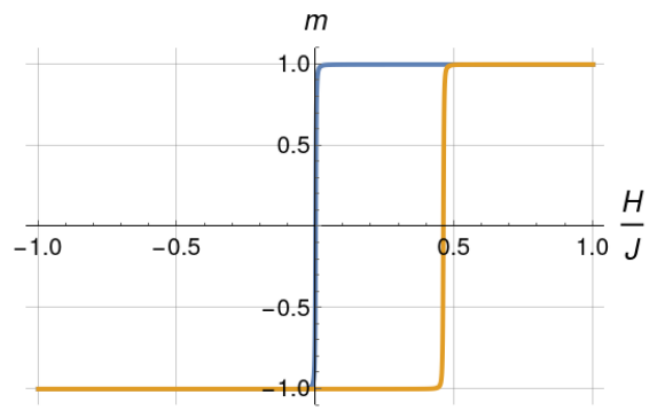

(d)

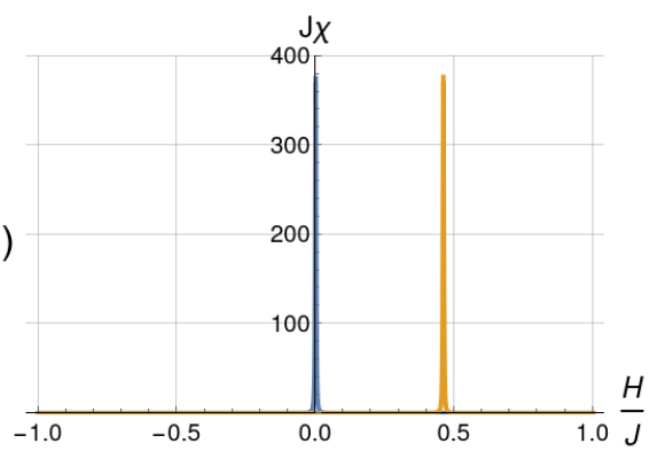

Figura 4.5: O gráfico (a) mostra a entropia por partícula em função do campo magnético, em (b) a magnetização por partícula em função do campo magnético, em (c) o calor específico a campo constante em função do campo magnético e em (d) a susceptibilidade em função do campo magnético. Em todas as figuras a curva azul representa o modelo de Ising enquanto a curva amarela representa o modelo de Ising com degenerescência $\omega=10$, ambos a temperatura $T k_{B} / J=0.4$.
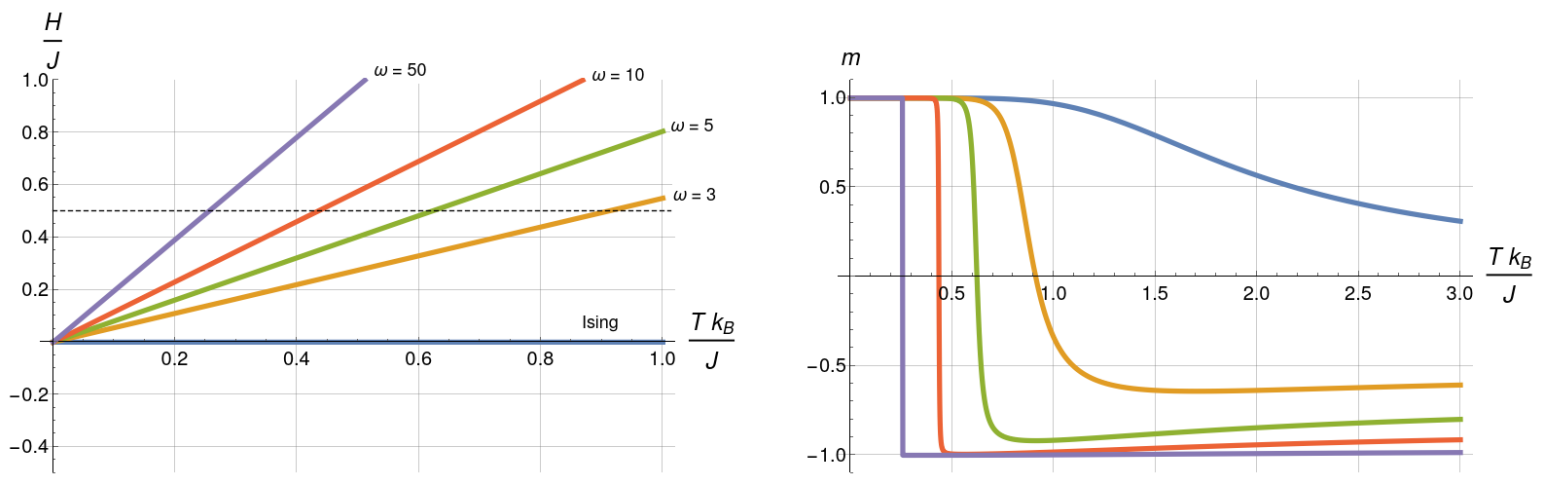

Figura 4.6: À esquerda, o diagrama de quase-fases para as degenerescências 1 (Ising), 3, 5, 10 e 50, cujas cores correspondem às degenerescências do gráfico à direita, da magnetização por partícula em função da temperatura. A linha tracejada no diagrama se refere ao campo $H / J=0.5$ que é tomado fixo no gráfico da magnetização. 
lidade, como a magnetização, não altera o seu formato, apenas o desloca, mostrando que o sistema exige mais intensidade de campo externo para "transicionar".

Essa análise, em conjunto com os resultados da figura (4.6), nos permitem sublinhar duas conclusões: em primeiro lugar, o modelo de Ising também apresenta pseudo-transições, conquanto ela não seja comumente observada, já que tradicionalmente estudamos como o sistema se comporta sob variação de temperatura, o que não permite o cruzamento da pseudo-transição, paralela ao eixo da temperatura - de fato, a figura (4.5) indica os sinais de pseudo-transição sob variação de campo. Sendo assim, o que nos possibilitou observar esse comportamento foi a degenerescência, posto que esta induz uma inclinação na linha de pseudo-coexistência com relação ao eixo horizontal, permitindo seu cruzamento sob variação de temperatura, para certos valores de campo fixo.

Em segundo lugar, esses resultados sugerem que o principal parâmetro responsável por quão contrastante são as quase-fases adjacentes é a temperatura. Se olharmos a magnetização e a susceptibilidade na figura (4.5), vemos que o comportamento dos dois sistemas é idêntico, salvo um deslocamento no valor de campo onde ocorre a mudança. Da mesma forma, na figura (4.6), vemos que, quanto maior é a inclinação do diagrama de quase-fases - como consequência da degenerescência - , menor é a temperatura em que a linha de pseudo-coexistência será cruzada, forçando um comportamento de quase-transição mais intenso.

\section{Entropia residual}

Cumpre fazermos ainda um comentário sobre entropia residual. Na figura (4.7) esquematizamos os diferentes caminhos possíveis para obter a entropia residual crítica. Vemos que há três caminhos possíveis: por cima da linha de pseudo-coexistência, por baixo ou sobre ela. No gráfico (d), mostramos uma varredura para diferentes ângulos de incidência, $\alpha$, sobre o ponto crítico, cujo comportamento está de acordo com a expressão (4.51).

Ainda que não seja possível ver a descontinuidade no gráfico, podemos olhar para a expressão da entropia residual em função do ângulo, obtida a partir da substituição de

$$
H(\alpha)=\frac{\alpha k_{B} T}{2} \ln \omega
$$


(a)

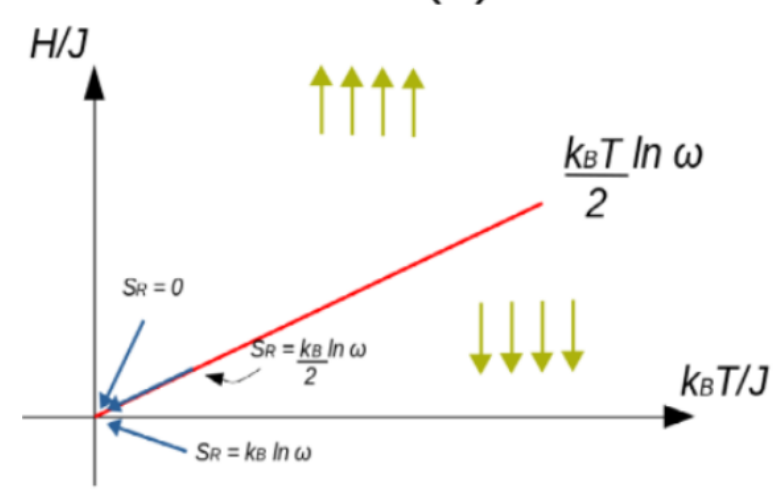

(c)

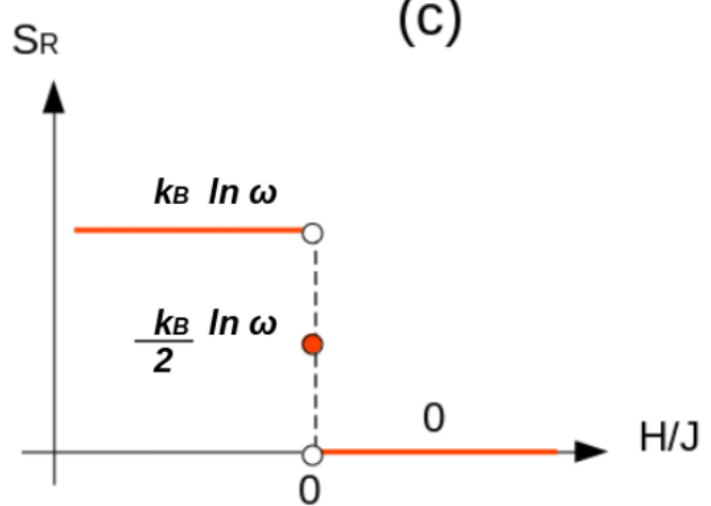

(b)

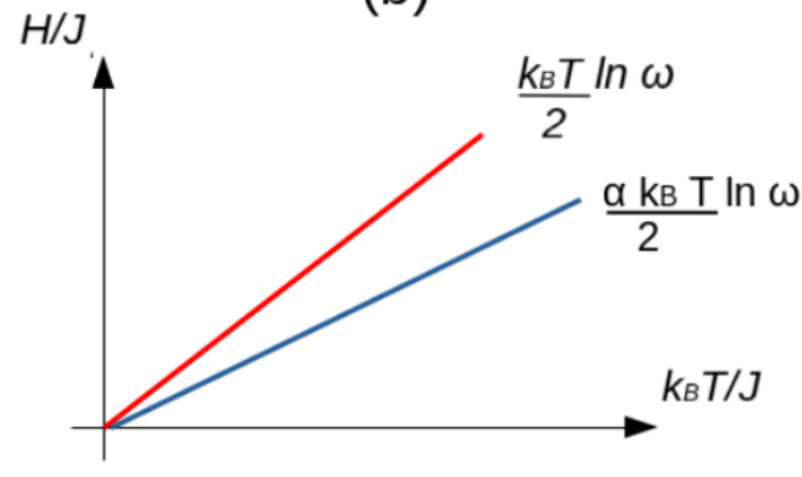

(d)

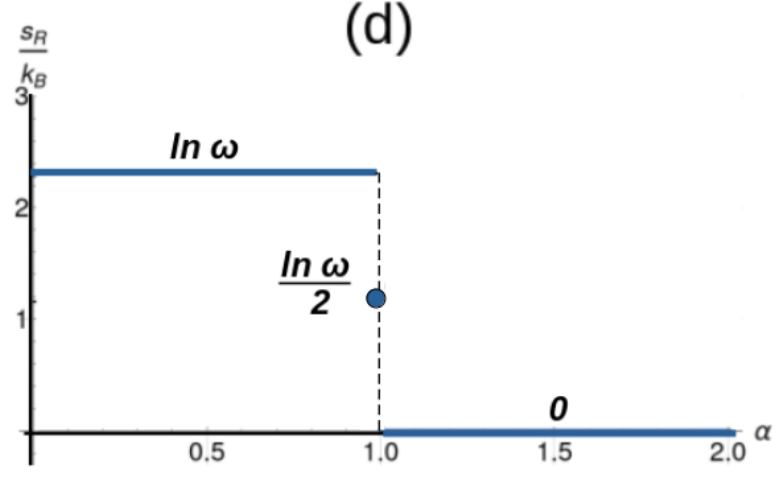

Figura 4.7: Em (a), ilustração esquemática do diagrama de quase-fases com os valores da entropia tomada no limite $T \rightarrow 0$ por diferentes caminhos. Em (b), a linha vermelha representa a curva pseudo-transição e a linha azul representa a mesma curva a menos de uma constante multiplicativa $\alpha$, isto é, com um coeficiente ângular distinto para qualquer $\alpha \neq 1$. Em (c), esquema da entropia residual em função do campo para os caminhos indicados no gráfico (a). Em (d), a entropia residual em função do ângulo $\alpha$, calculada para a linha azul do gráfico (b). 
na expressão da entropia (4.39), tomando o limite $T \rightarrow 0$

$$
S_{R}(\alpha)=\frac{\alpha \ln \omega}{2}\left(1-\frac{1-\omega^{1-\alpha}}{\left|1-\omega^{1-\alpha}\right|}\right)+\ln \left(\frac{1+\omega^{1-\alpha}+\left|1-\omega^{1-\alpha}\right|}{2}\right)
$$

ou, de forma equivalente

$$
S_{R}(\alpha)= \begin{cases}\ln \omega, & \alpha<1, \\ \frac{\ln \omega}{2}, & \alpha=1 \\ 0, & \alpha>1 .\end{cases}
$$

Esse resultado nos mostra um desacordo com a conjectura de Rojas [21] de que a continuidade da entropia residual na fronteira com ao menos uma das fases adjacentes sinaliza a existência de uma pseudo-transição, figura (1.4). Por um lado, talvez a continuidade da entropia residual seja um critério suficente, mas não necessário, para garantir a manifestação da pseudotransição. Por outro lado, o caso estudado aqui não se enquadra nos possíveis cenários que o artigo apresenta, pois a hipótese é que a entropia residual na fronteira será, em geral, maior que as das fases adjacentes, o que não ocorre no modelo de Ising $\omega$-degenerado, como nos conta a equação (4.58).

Por fim, como adiantamos na introdução, um ponto que se manteve em estado de interrogação foi a questão da definição de entropia residual crítica. Não encontramos na literatura estudos que se dedicam a esse conceito - salvo alguns que encontramos, mas que esbarram na questão sem se aprofundar nela, alguns exemplos são [40, 41, 42]. Dado que o valor da entropia, no limite $T \rightarrow 0$, em direção ao ponto crítico, depende do caminho a ser tomado, não ficou claro para nós qual nos fornece, de fato, a entropia residual crítica. Nos nossos cálculos, assumimos que essa quantidade é aquela tomada sobre a pseudo-transição, mas não por uma definição matemática formal e sim por uma razoabilidade teórica devido à simetria do sistema.

\section{Comprimento de correlação}

O comprimento de correlação entre os spins do sistema (cap. 2 de [4]), é dado por 


$$
\xi=\left[\ln \left(\frac{\lambda_{+}}{\lambda_{-}}\right)\right]^{-1}
$$

onde $\lambda_{ \pm}$são dados por (4.36). Explicitamente, temos

$$
\xi(\beta, H ; \omega)=\left\{\ln \left[\frac{e^{\beta H}+\omega e^{-\beta H}+\sqrt{\left(e^{\beta H}-\omega e^{-\beta H}\right)^{2}+4 \omega e^{-4 \beta J}}}{e^{\beta H}+\omega e^{-\beta H}-\sqrt{\left(e^{\beta H}-\omega e^{-\beta H}\right)^{2}+4 \omega e^{-4 \beta J}}}\right]\right\}^{-1} .
$$

No limite para baixas temperaturas, $t \ll 1$, é interessante notar que o valor depende do campo externo

$$
\xi(t \ll 1, H ; \omega)= \begin{cases}\frac{1}{\ln \omega}, & H=0, \\ \infty, & H=\frac{k_{B} T \ln \omega}{2}, \\ 0, & H \neq 0 \text { e } H \neq \frac{k_{B} T \ln \omega}{2} .\end{cases}
$$

Mostramos alguns resultados na figura (4.8).

No gráfico (b), vemos que $\xi$ se comporta de forma similar ao que ocorre com a susceptibilidade em função do campo na figura (4.5), deslocando o pico sem alterar o seu tamanho, sugerindo novamente que o grau de contraste entre as quase-fases depende somente da temperatura. A linha pontilhada lilás se refere ao valor calculado sobre a pseudo-transição, independente de qual degenerescência.

No gráfico (c), a campo nulo, duas características merecem destaque: primeiro, o fato de o comprimento de correlação para o modelo de Ising estar sobreposto ao comprimento de correlação sobre a pseudo-coexistência, o que é esperado já que, como é possível ver no diagrama (a), a linha $H / J=0$ é precisamente a pseudo-transição. Em segundo lugar, ainda sobre o gráfico (c), notamos que $\xi$ não tende a zero quando o sistema é resfriado, diferentemente do que ocorre na presença de campo externo, gráfico (d). Na verdade, seus valores convergem segundo indica a expressão (4.61). O fato de haver uma divergência no comprimento de correlação ao nos aproximarmos da origem do diagrama sobre a linha de pseudo-transição está em acordo com o fato de este ponto ser crítico no modelo de Ising unidimensional. O que é curioso, entretanto, é que o mesmo limite, tomado sobre $H / J=0, \operatorname{com} \omega \neq 0$, faz $\xi$ convergir para $1 / \ln \omega$. 

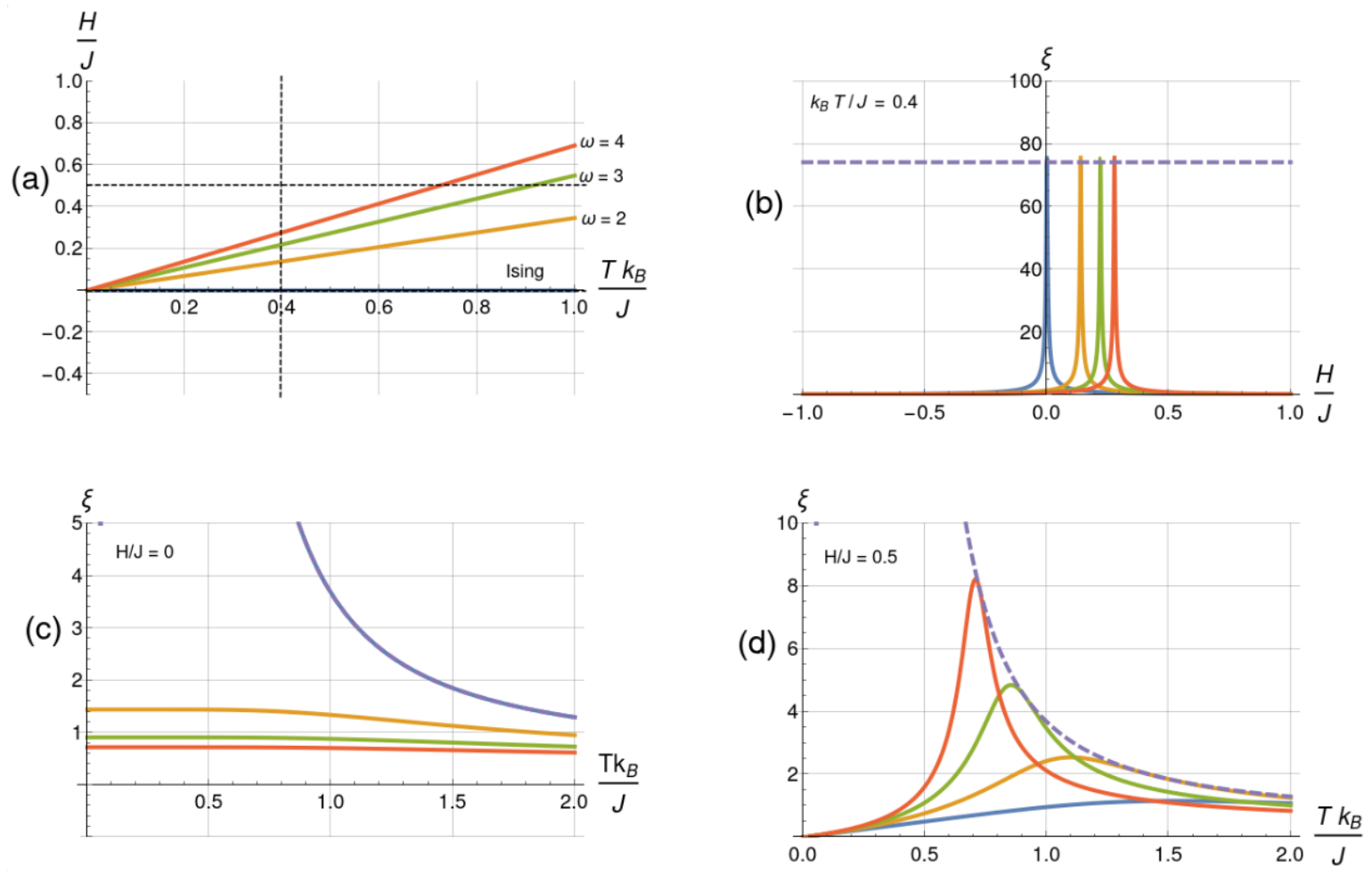

Figura 4.8: Em (a), o diagrama de quase-fases do modelo de Ising para as degenerescências $\omega=1,2,3$ e 4, que representam os comprimentos de correlação das outras figuras, as cores das curvas são correspondentes. As linhas tracejadas são os valores fixos de temperatura $k_{B} T / J=0.4$ e campos externos $H / J=0$ e 0.5 nos gráficos (b), (c) e (d), respectivamente. Em (b), o comprimento de correlação em função do campo magnético, a linha tracejada lilás é o comprimento de correlação calculado sobre a linha de pseudo-coexistência. Em (c), o comprimento de correlação em função da temperatura para campo nulo. Em (d), o comprimento de correlação em função da temperatura para $H / J=0.5$, novamente a linha tracejada é valor sobre a pseudo-coexistência. 
Por fim, o gráfico (d) indica novamente o papel importante da temperatura nas pseudotransições. Notamos que há um máximo no comprimento de correlação na pseudo-transição, que é tanto maior quanto menor é a temperatura em que essa linha é cruzada.

Como foi possível notar, o caminho tomado para cruzar as linhas de pseudo-transição, em geral, interfere nos resultados — bem como no cálculo dos limites. Por esse motivo, vamos analisar o comportamento de $\xi, m$ e $s$ ao cruzar a linha de pseudo-coexistência perpendicularmente. Para isso, vamos obter uma família de retas perpendiculares à reta (4.54), isto é, uma função $H_{t}(T, \omega)$, tal que $H$ e $H_{t}$ são perpendiculares. A expressão, adimensional, que queremos é

$$
\hat{H}_{t}(T ; \omega)=-\frac{2}{\ln \omega} t+d \sqrt{\left(\frac{2}{\ln \omega}\right)^{2}+1}
$$

onde $\hat{H}_{t}=H_{t} / J$ e $d$ é a distância que vai da intersecção de $H$ e $H_{t}$ até a origem do diagrama.
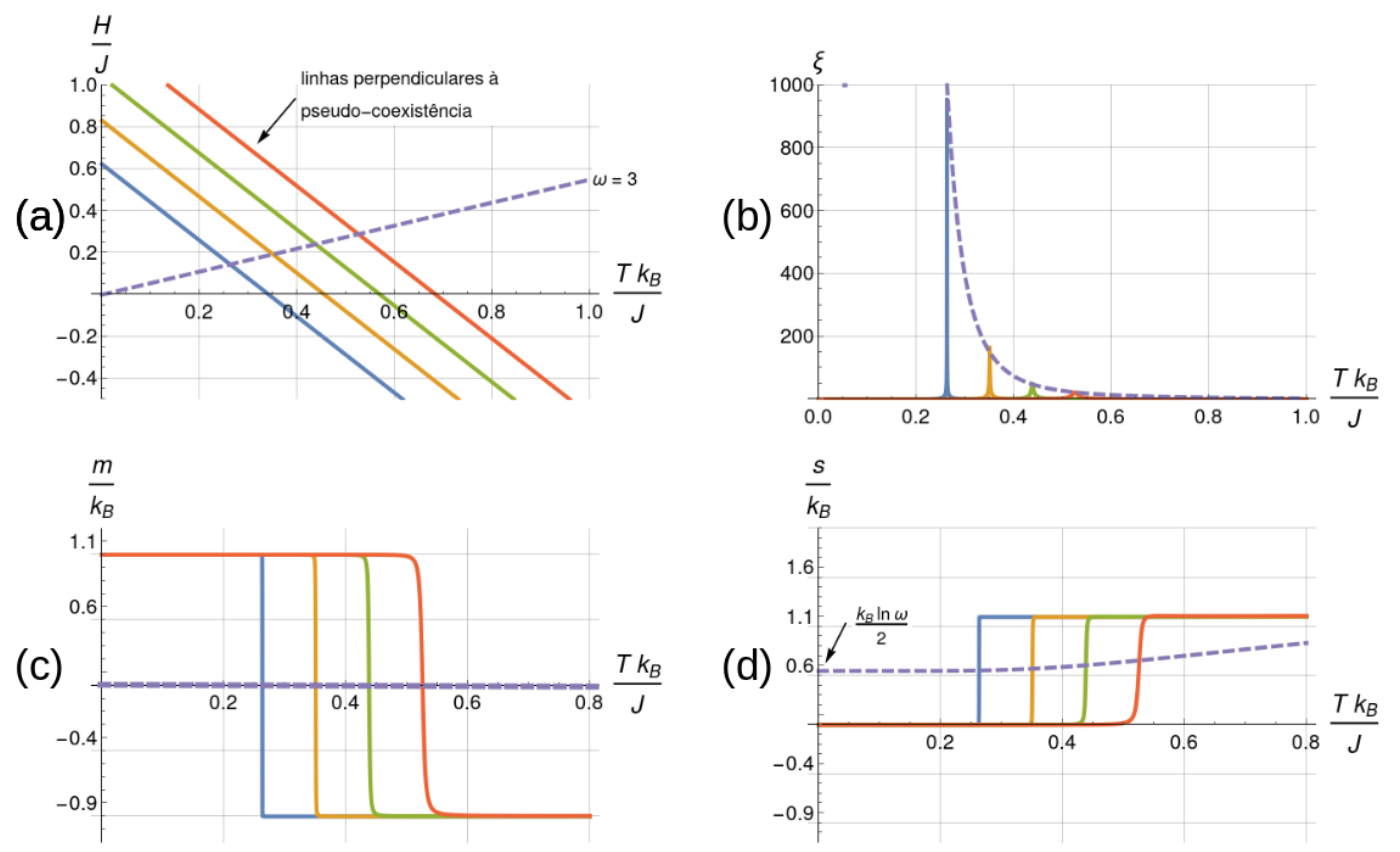

Figura 4.9: Em (a), o diagrama de quase-fases cuja linha de coexistência é a tracejada e as demais são perpendiculares a esta. As cores das curvas de todos os gráficos são correspondentes, representando os caminhos seguidos. Em (b), o comprimento de correlação em função da temperatura. Em (c), a magnetização em função da temperatura. Em (d), entropia em função da temperatura.

O comprimento de correlação se comporta de forma similar ao que ocorre a campo fixo não nulo, no gráfico (d) da figura (4.8), mas de forma mais abrupta, mostrando somente um pico fino muito próximo à pseudo-transição. As demais quantidades exibem comportamentos similares para os quatro pontos de pseudo-transição, com a mudança se tornando levemente mais abrupta 
conforme a temperatura diminui. Note que a magnetização é nula sobre a pseudo-coexistência, para todos os valores de $T$, o que pode ser obtido calculando a magnetização (4.41) sob a condição (4.54). Veja, também, que a entropia calculada sobre a quase-fronteira converge para $\frac{k_{B} \ln \omega}{2}$, no limite $T \rightarrow 0$, como já discutimos.

Por fim, um último resultado interessante que gostaríamos de apresentar é a diferença entre a magnetização nas proximidades da pseudo-transição. Vamos considerar duas linhas paralelas a esta, a uma distância $\delta$, como indicado na figura (4.10). As linhas pontilhadas, acima e abaixo da quase-fronteira, são dadas por

$$
H_{ \pm}=\frac{k_{B} T \ln \omega}{2} \pm \delta
$$

Substituindo essa relação na expressão da magnetização (4.41), podemos obter a diferença entre essas quantidades nas duas quase-fases adjacentes

$$
\Delta m=m_{+}-m_{-}=\frac{\sinh (\beta \delta)}{\sqrt{\sinh ^{2}(\beta \delta)+e^{-4 \beta J}}}
$$

cujo gráfico para um valor $\delta / J=0.01$ pode ser visto na figura (4.10)
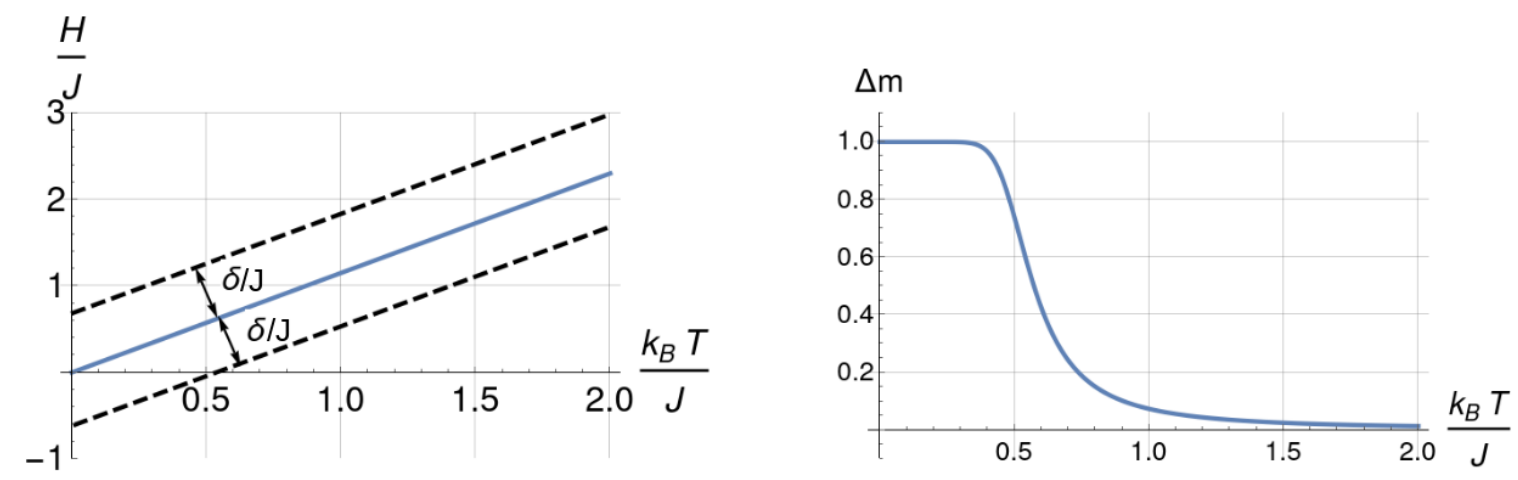

Figura 4.10: À esquerda, a linha de pseudo-coexistência do modelo de Ising degenerado, em azul, e linhas paralelas a esta tracejada, a uma distância $\delta$, acima e abaixo. À direita, a diferença entre as magnetizações sobre as linhas pontilhadas, em função da temperatura.

Primeiro, notamos que a equação (4.64) depende somente da temperatura e da distância até a pseudo-transição. Com efeito, vimos que a degenerescência afeta o sistema na medida em que aproxima a linha de pseudo-coexistência da região de temperatura baixa.

Segundo, sublinhamos o fato de $\Delta m$ se manter constante, aproximadamente igual a 1 , até 
uma determinada temperatura, seguida de uma queda rápida. O final deste patamar inicial pode ser a sugestão da existência de um pseudo-ponto crítico, onde a linha que separa as quase-fases se desfaz - e aparentemente seria apenas essa a função deste ponto, posto que o sinal de uma transição contínua, a divergência (ou a descontinuidade) nas segundas derivadas da energia livre já é observada em toda a quase-fronteira. Um comportamento nesse sentido pode ser observado para o diagrama de densidade de magnetização, na figura (4.3): seguindo a pseudo-transição, quando $k_{B} T / J \approx 1$, a linha se dissipa em uma região de magnetizações entre -0.5 e 0,5.

\subsection{Fluido de rede unidimensional}

Vamos dedicar um espaço para resolver o fluido de rede unidimensional, cuja Hamiltoniana é dada por (3.27), numa cadeia com $L$ sítios e $N$ partículas, fazendo novamente uso do método da matriz de transferência. A "Hamiltoniana"efetiva, com a contribuição da flutuação de partículas, unidimensional, é dada por

$$
\mathcal{H}_{e f}(\{\eta\})=-\epsilon \sum_{i=1}^{L} \eta_{i} \eta_{i+1}-\mu \sum_{i=1}^{L} \eta_{i}
$$

Agora estamos no ensemble grande canônico, $(T, L, \mu)$. A energia livre, dada pelo grande potencial termodinâmico, $\Phi=\Phi(T, L, \mu)$, pode ser obtido da transformada de Legendre da energia livre de Helmholtz, $F=F(T, L, N)$ com relação à variável $N$

$$
\Phi=F-\mu N
$$

Diferenciando a função (4.66) e usando a diferencial (3.20), obtemos

$$
d \Phi=-S d T-\sigma d L-N d \mu
$$

onde $\sigma$ é a tensão linear, equivalente a uma pressão em uma dimensão. A identificação com os termos que aparecem na diferencial de $\Phi$ nos fornece as equações de estado

$$
S=-\left(\frac{\partial \Phi}{\partial T}\right)_{L, \mu}, \quad \sigma=-\left(\frac{\partial \Phi}{\partial L}\right)_{T, \mu}, \quad N=-\left(\frac{\partial \Phi}{\partial \mu}\right)_{T, L}
$$


A grande função de partição será

$$
\mathcal{Z}(T, L, \mu)=\sum_{\{\eta\}} e^{-\beta[\mathcal{H}(\{\eta\})-\mu N]}
$$

Os elementos de matriz, simetrizados, são

$$
T\left(\eta_{i}, \eta_{i+1}\right)=\exp \left[\beta \epsilon \eta_{i} \eta_{i+1}+\frac{\beta \mu}{2}\left(\eta_{i}+\eta_{i+1}\right)\right]
$$

de maneira que a matriz de transferência do fluido de rede é

$$
\mathbb{T}=\left[\begin{array}{cc}
T(0,0) & T(0,1) \\
T(1,0) & T(1,1)
\end{array}\right]=\left[\begin{array}{cc}
1 & e^{\frac{\beta \mu}{2}} \\
e^{\frac{\beta \mu}{2}} & e^{\beta(\epsilon+\mu)}
\end{array}\right]
$$

e a equação (4.69) pode ser reescrita da forma compacta

$$
\begin{gathered}
\mathcal{Z}(T, L, \mu)=\sum_{\{\eta\}} \prod_{i=1}^{L} T\left(\eta_{i}, \eta_{i+1}\right)=\operatorname{tr}\left(\mathbb{T}^{L}\right), \\
\mathcal{Z}(T, L, \mu)=\lambda_{+}^{L}+\lambda_{-}^{L} .
\end{gathered}
$$

Os autovalores da matriz são obtidos novamente da equação secular (4.9), que nos fornece

$$
\lambda_{ \pm}=\frac{e^{\beta(\epsilon+\mu)}+1 \pm \sqrt{\left[e^{\beta(\epsilon+\mu)}-1\right]^{2}+4 e^{\beta \mu}}}{2}
$$

Ora, sendo $\lambda_{+}>\lambda_{-}$, temos

$$
\mathcal{Z}(T, L, \mu)=\lambda_{+}^{L}=\left\{\frac{e^{\beta(\epsilon+\mu)}+1+\sqrt{\left[e^{\beta(\epsilon+\mu)}-1\right]^{2}+4 e^{\beta \mu}}}{2}\right\}^{L}
$$

O grande potencial termodinâmico é definido de forma equivalente à feita nos outros ensembles, (3.22) e (4.16)

$$
\Phi(T, L, \mu)=-k_{B} T \ln \mathcal{Z}(T, \mu, L),
$$

de sorte que a energia livre por sítio será 


$$
\phi(T, \mu)=\frac{\Phi(T, L, \mu)}{L}=-k_{B} T \ln \left\{\frac{e^{\beta(\epsilon+\mu)}+1+\sqrt{\left[e^{\beta(\epsilon+\mu)}-1\right]^{2}+4 e^{\beta \mu}}}{2}\right\}
$$

Vamos determinar uma expressão para o número médio de partículas

$$
\begin{gathered}
\mathcal{Z}=\sum_{\alpha} e^{-\beta\left(\mathcal{H}_{\alpha}-\mu N_{\alpha}\right)} \Longrightarrow\left(\frac{\partial \mathcal{Z}}{\partial \mu}\right)_{T, L}=\beta \sum_{\alpha} N_{\alpha} e^{-\beta\left(\mathcal{H}_{\alpha}-\mu N_{\alpha}\right)} \\
\frac{1}{\mathcal{Z}\left(\frac{\partial \mathcal{Z}}{\partial \mu}\right)_{T, L}}=\frac{\beta}{\mathcal{Z}} \sum_{\alpha} N_{\alpha} e^{-\beta\left(\mathcal{H}_{\alpha}-\mu N_{\alpha}\right)}=\beta\langle N\rangle \\
\langle N\rangle=\frac{1}{\beta}\left(\frac{\partial \ln \mathcal{Z}}{\partial \mu}\right)_{T, L} .
\end{gathered}
$$

Por conseguinte, podemos definir a densidade da cadeia como

$$
\begin{gathered}
\rho(T, \mu):=\frac{\langle N\rangle}{L}=\frac{k_{B} T}{L}\left[\frac{\partial \ln \phi(T, \mu)}{\partial \mu}\right]_{T}, \\
\rho(T, \mu)=\frac{k_{B} T}{\lambda_{+}}\left(\frac{\partial \lambda_{+}}{\partial \mu}\right)_{\beta} .
\end{gathered}
$$

Do mesmo modo, vamos determinar uma expressão para a energia interna

$$
\begin{gathered}
\mathcal{Z}=\sum_{\alpha} e^{-\beta\left(\mathcal{H}_{\alpha}-\mu N_{\alpha}\right)} \Longrightarrow\left(\frac{\partial \mathcal{Z}}{\partial \beta}\right)_{\mu, L}=-\sum_{\alpha}\left(\mathcal{H}_{\alpha}-\mu N_{\alpha}\right) e^{-\beta\left(\mathcal{H}_{\alpha}-\mu N_{\alpha}\right)} \\
\frac{1}{\mathcal{Z}}\left(\frac{\partial \mathcal{Z}}{\partial \beta}\right)_{\mu, L}=-\frac{1}{\mathcal{Z}} \sum_{\alpha} \mathcal{H}_{\alpha} e^{-\beta\left(\mathcal{H}_{\alpha}-\mu N \alpha\right)}+\frac{\mu}{\mathcal{Z}} \sum_{\alpha} N_{\alpha} e^{-\beta\left(\mathcal{H}_{\alpha}-\mu N \alpha\right)} \\
\left(\frac{\partial \ln \mathcal{Z}}{\partial \beta}\right)_{\mu, L}=-U+\mu\langle N\rangle \\
U(T, \mu, L)=\mu\langle N\rangle(T, \mu, L)-\left[\frac{\partial \ln \mathcal{Z}(T, \mu, L)}{\partial \beta}\right]_{\mu, L}
\end{gathered}
$$


onde $U=\langle\mathcal{H}\rangle$. Segue que a energia interna por sítio, em termos do maior autovalor, é

$$
u(T, \mu)=\mu \rho(T, \mu)-\frac{1}{\lambda_{+}}\left(\frac{\partial \lambda_{+}}{\partial \beta}\right)_{\mu} .
$$

Explicitamente, a densidade e a energia interna por sítio são dadas, respectivamente, por

$$
\begin{aligned}
& \rho(T, \mu)=\frac{e^{\beta \mu}}{e^{\beta(\mu+\epsilon)}+1+\sqrt{\left[e^{\beta(\mu+\epsilon)}-1\right]^{2}+4 e^{\beta \mu}}}\left\{e^{\beta \epsilon}+\frac{\left[e^{\beta(\mu+\epsilon)}-1\right] e^{\beta \epsilon}+2}{\sqrt{\left[e^{\beta(\mu+\epsilon)}-1\right]^{2}+4 e^{\beta \mu}}}\right\}, \\
& u(T, \mu)=-\frac{\epsilon e^{\beta(\mu+\epsilon)}}{e^{\beta(\mu+\epsilon)}+1+\sqrt{\left.\left[e^{\beta(\mu+\epsilon)}-1\right)\right]^{2}+4 e^{\beta \mu}}}\left\{1+\frac{e^{\beta(\mu+\epsilon)}-1}{\sqrt{\left[e^{\beta(\mu+\epsilon)}-1\right]^{2}+4 e^{\beta \mu}}}\right\},
\end{aligned}
$$

Apresentamos alguns resultados na figura (4.11)

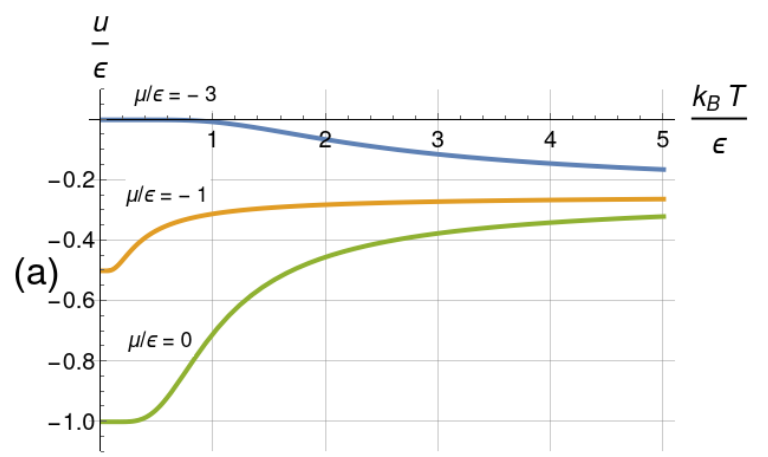

(b)

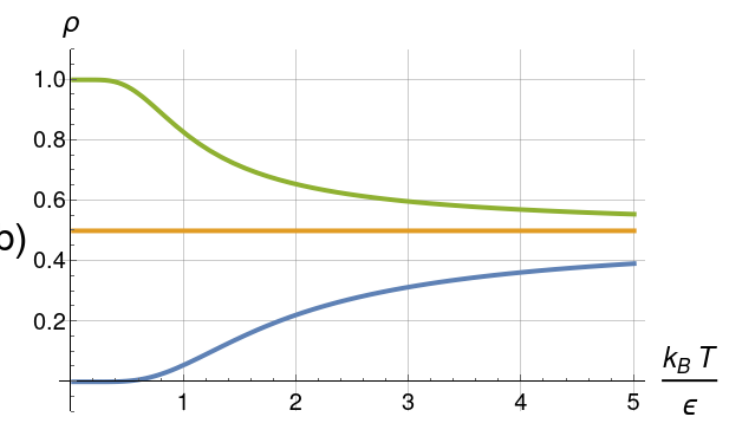

(c)
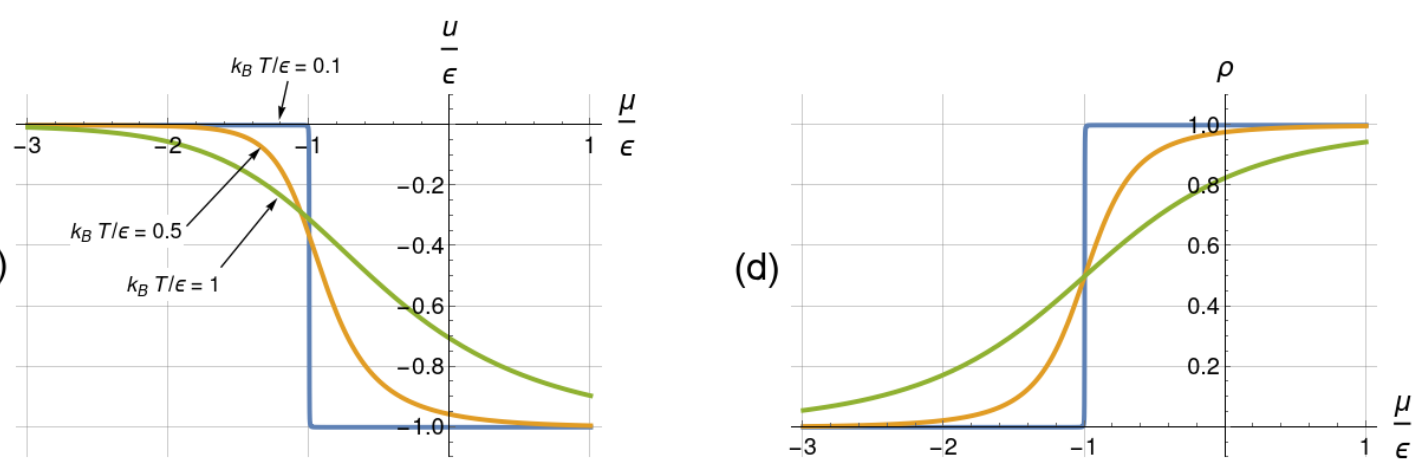

Figura 4.11: Em (a), a energia interna por sítio em função da temperatura, para os potenciais químicos $\mu / \epsilon=-3,-1$ e 0 , cujas cores das curvas são as mesmas para os potenciais do gráfico (b), da densidade em função da temperatura. Em (c), a energia interna por sítio em função do potencial químico, para as temperaturas $k_{B} T / \epsilon=0.1,0.5$ e 1 , cujas cores são as mesmas para as temperaturas do gráfico (d), da densidade em função do potencial químico

Dos gráficos (a) e (b) vemos como o potencial químico se relaciona ao quão preenchida está 
a rede. À temperatura próxima a zero está preenchida para $\mu / \epsilon=0$ e vazia para $\mu / \epsilon=-3$, com energias por partícula $u / \epsilon=-1$ e 0 , respectivamente. Por outro lado, para altas temperaturas a rede converge para um estado semi-preenchido, $\operatorname{com} \rho=1 / 2$ e a energia por sítio para $u / \epsilon=-1 / 4$.

Outro ponto que vale destacar é o comportamento do sistema quando $\mu / \epsilon=-1$, que fixa a rede num estado semi-preenchido para qualquer temperatura. Vemos nos gráficos (c) e (d) que esse valor de potencial químico se manifesta de maneira muito similar a uma pseudo-transição, se acentuando cada vez mais para temperaturas baixas. É possível ver na figura (c) como a curva para $k_{B} T / \epsilon=0.1$ se assemelha muito a uma função degrau. Com efeito, se fizermos uso da relação entre o campo externo e o potencial químico (3.34), obtido do mapeamento (3.28), vemos que a quase-fronteira do modelo de Ising unidimensional, em $H / J=0$, corresponde a uma linha para o fluido de rede unidimensional em $\mu / \epsilon=-1$. Não apenas isso, as expressões obtidas aqui, (4.82) e (4.81), poderiam também ser deduzidas a partir das expressões do modelo de Ising (4.40) e (4.41), respectivamente, através dos mapeamentos (3.28), (3.30) e (3.34).

\subsection{Fluido de rede unidimensional degenerado}

Seguindo o mesmo roteiro, vamos adicionar uma degenerescência $\omega$ ao fluido de rede, no estado $\eta=1$ - que pode ser pensado como um lipídio na rede com $\omega$ possíveis dobras nas caudas apolares, de fato, lembramos que o modelo de Doniach é um sistema de dois estados com degenerescência em um deles. Pelo mesmo argumento usado para construir a "Hamiltoniana"efetiva do modelo de Ising $\omega$-degenerado (4.33), aqui obtemos, já simetrizada

$$
\mathcal{H}(\{\eta\})=-\epsilon \sum_{i=1}^{L} \eta_{i} \eta_{i+1}-\frac{\mu}{2} \sum_{i=1}^{L}\left(\eta_{i}+\eta_{i+1}\right)-\frac{\ln \omega}{2 \beta} \sum_{i=1}^{L}\left(\eta_{i}+\eta_{i+1}\right) .
$$

Destarte, a matriz de transferência é dada por

$$
\mathbb{T}=\left[\begin{array}{cc}
T(0,0) & T(0,1) \\
T(1,0) & T(1,1)
\end{array}\right]=\left[\begin{array}{cc}
1 & \sqrt{\omega z} \\
\sqrt{\omega z} & \omega z e^{\beta \epsilon}
\end{array}\right],
$$

onde $z:=e^{\beta \mu}$ é a fugacidade, usada aqui por motivos de simplicidade na notação. Os autova- 
lores de (4.84) são

$$
\lambda_{ \pm}=\frac{1+\omega z e^{\beta \epsilon} \pm \sqrt{\left(1-\omega z e^{\beta \epsilon}\right)^{2}+4 \omega z}}{2}
$$

de maneira que a grande função de partição é

$$
\mathcal{Z}(T, \mu ; \omega)=\left\{\frac{1+\omega e^{\beta(\epsilon+\mu)} \pm \sqrt{\left[1-\omega e^{\beta(\epsilon+\mu)}\right]^{2}+4 \omega e^{\beta \mu}}}{2}\right\}^{L}
$$

e o grande potencial termodinâmico por sítio

$$
\phi(T, \mu, \omega)=-k_{B} T \ln \left\{\frac{1+\omega e^{\beta(\epsilon+\mu)} \pm \sqrt{\left[1-\omega e^{\beta(\epsilon+\mu)}\right]^{2}+4 \omega e^{\beta \mu}}}{2}\right\} .
$$

A densidade e a energia interna por sítio são obtidas através de, respectivamente, (4.78) e (4.80)

$$
\rho(T, \mu ; \omega)=\frac{\omega e^{\beta \mu}}{1+\omega e^{\beta(\epsilon+\mu)}+\sqrt{\left[1-\omega e^{\beta(\epsilon+\mu)}\right]^{2}+4 \omega e^{\beta \mu}}}\left\{e^{\beta \epsilon}+\frac{\left[\omega e^{\beta(\epsilon+\mu)}-1\right] e^{\beta \epsilon}+2}{\sqrt{\left[1-\omega e^{\beta(\epsilon+\mu)}\right]^{2}+4 \omega e^{\beta \mu}}}\right\},
$$

$\mathrm{e}$

$$
u(T, \mu ; \omega)=-\frac{\epsilon \omega e^{\beta(\epsilon+\mu)}}{1+\omega e^{\beta(\epsilon+\mu)}+\sqrt{\left[1-\omega e^{\beta(\epsilon+\mu)}\right]^{2}+4 \omega e^{\beta \mu}}}\left\{1+\frac{\omega e^{\beta(\epsilon+\mu)}-1}{\sqrt{\left[1-\omega e^{\beta(\epsilon+\mu)}\right]^{2}+4 \omega e^{\beta \mu}}}\right\} .
$$

Além disso, a entropia por sítio pode ser calculada através da equação de estado dada em (4.68)

$$
s(T, \mu ; \omega)=-\left[\frac{\partial \phi(T, \mu ; \omega)}{\partial T}\right]_{\mu}=\frac{k_{B}}{L} \ln \mathcal{Z}(T, \mu ; \omega)+\frac{k_{B} T}{L}\left[\frac{\ln \mathcal{Z}(T, \mu ; \omega)}{\partial T}\right]_{\mu} .
$$

Em termos do maior autovalor e da derivada em relação à $\beta$ 


$$
s(T, \mu ; \omega)=k_{B} \ln \lambda_{+}-\frac{1}{T} \frac{1}{\lambda_{+}}\left(\frac{\partial \lambda_{+}}{\partial \beta}\right)_{\mu},
$$

ou, mais explicitamente

$$
s(T, \mu ; \omega)=k_{B} \ln \lambda_{+}-\frac{1}{T} \frac{\omega e^{\beta \mu}}{2 \lambda_{+}}\left\{e^{\beta \epsilon}(\mu+\epsilon)+\frac{\omega(\mu+\epsilon) e^{\beta(2 \epsilon+\mu)}+2 \mu}{\sqrt{\left[1-\omega e^{\beta(\epsilon+\mu)}\right]^{2}+4 \omega e^{\beta \mu}}}\right\} .
$$

Também é possível calcular a capacidade térmica por sítio a potencial químico constante através de

$$
c_{\mu}(T ; \omega)=T\left[\frac{\partial s(T, \mu ; \omega)}{\partial T}\right]_{\mu} .
$$

Se utilizarmos novamente o raciocínio de Rojas para obter a linha de pseudo-coexistência a partir dos elementos da diagonal da matriz de transferência (4.84), temos que a competição entre $T(0,0)$ e $T(1,1)$ implica em uma quase-fronteira em $T(1,1)=T(0,0)$, dada por

$$
\mu_{p}=k_{B} T \ln \omega-\epsilon
$$

Note que para o caso $\omega=1$, temos $\mu_{p}=-\epsilon$, como já vimos.

Substituindo essa relação na expressão da densidade (4.88), não é difícil concluir que o resultado é $\rho_{p}=1 / 2$, o que significa que, sobre a pseudo-coexistência, a rede está sempre semi-preenchida. No fluido de rede vimos que isso ocorre para $\mu / \epsilon=-1$, que é a pseudotransição naquele caso. Note também que essa condição é análoga ao que ocorre no modelo de Ising, que possui magnetização nula sobre esse linha.

Também é possível mostrar que sobre (4.93), a densidade (4.88) é sempre $\rho_{p}=1 / 2$, independentemente da temperatura, o mesmo que ocorria para a magnetização no modelo de Ising, que se anulava sobre a pseudo-coexistência. Aliás, vemos que esse comportamento também está em acordo com o mapeamento (3.30).

Alguns dos resultados podem ser observados nas figuras (4.12), (4.13) e (4.14).

Novamente é possível vermos o aparecimento do fenômeno de pseudo-transição, eviden- 


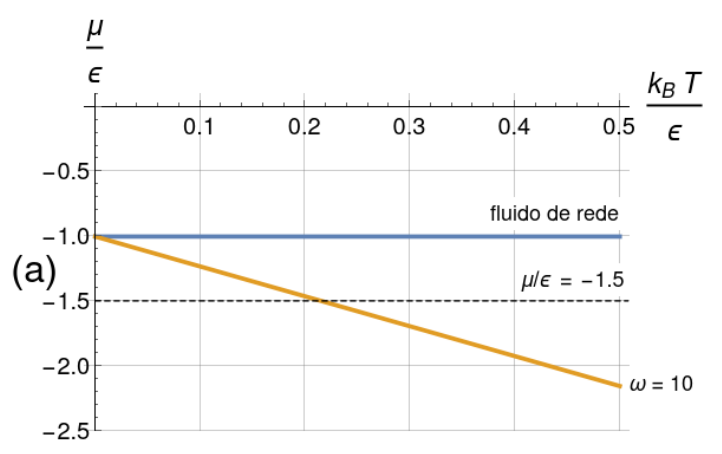

(b)
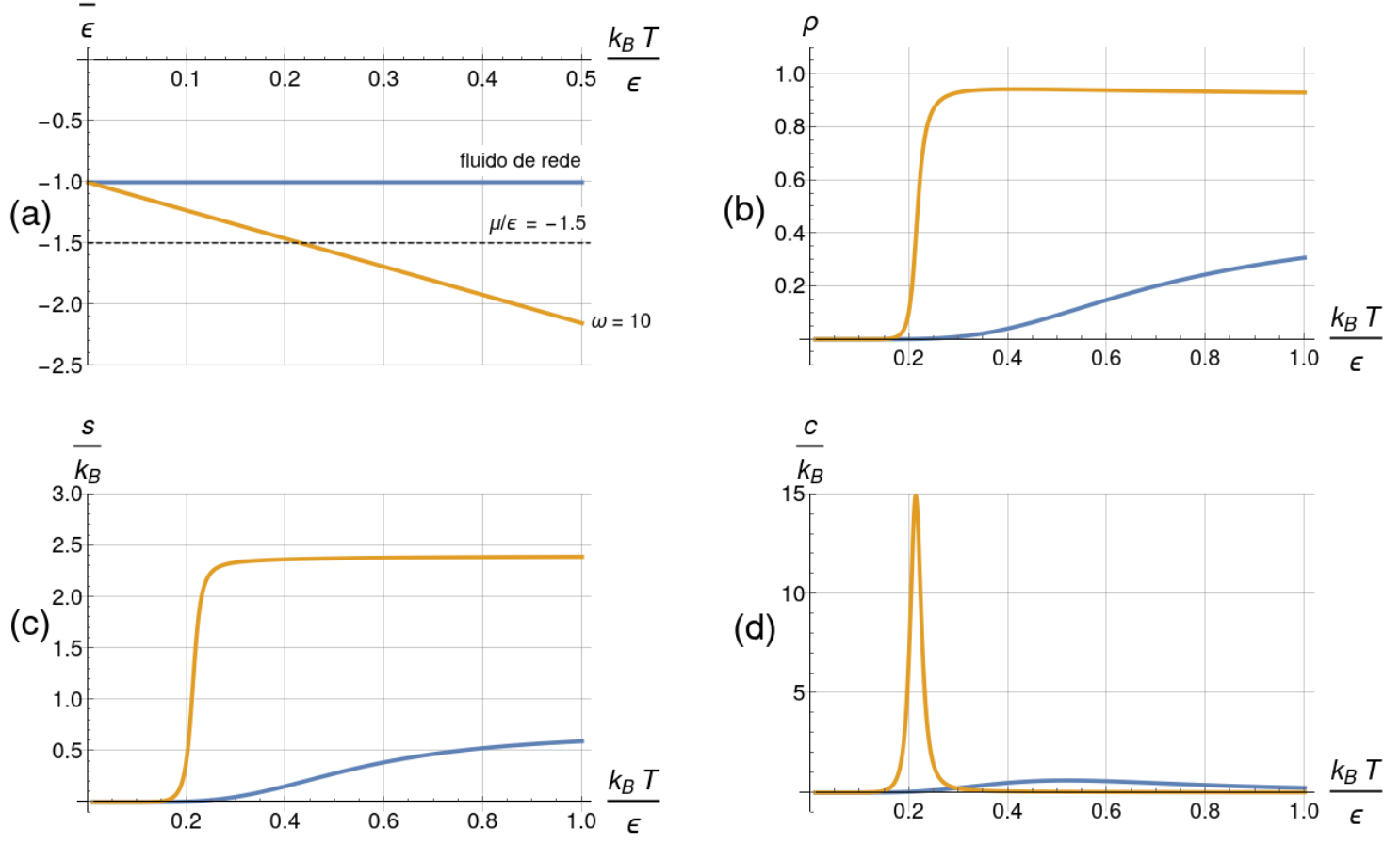

Figura 4.12: Em (a), o diagrama $\mu-T$ do fluido de rede, para o caso usual $(\omega=1)$ e o caso com degenerescência 10, a linha horizontal pontilhada, em $\mu / \epsilon=-1.5$, se refere ao potencial químico tomado fixo nos demais gráficos. O gráfico (b) mostra a densidade em função da temperatura. $\mathrm{O}$ gráfico (c) mostra a entropia por sítio em função da temperatura, e em (d) a capacidade térmica por sítio a potencial químico constante em função da temperatura.

ciado pela adição de degenerescência: uma mudança repentina das características do sistema, acompanhada de um pico acentuado na capacidade térmica por sítio.

Na figura (4.12), onde o potencial químico é mantido fixo em $\mu / \epsilon=-1.5$, é possível ver que o cruzamento da linha de pseudo-coexistência implica na passagem de uma região de baixa densidade, característica de uma quase-fase gasosa, para uma região com alta densidade, dita quase-fase fluida; essa transição é ainda seguida por um pico na capacidade térmica por sítio. $\mathrm{O}$ fluido de rede não degenerado não expressa sinais de pseudo-transição, não obstante o caminho tomado, para $\mu$ fixo, também não cruza linhas de pseudo-coexistência, não sendo um fenômeno realmente esperado.

Na figura (4.13), para a temperatura fixa $k_{B} T / \epsilon=0.2$, o cenário se repete no caso do fluido de rede degenerado, com as variações abruptas esperadas. Por outro lado, o fluido de rede usual apresenta uma mudança na densidade idêntica ao que ocorre no modelo degenerado, entretanto com pouco efeito sobre a entropia e a capacidade térmica por sítio.

Já na figura (4.14), mostramos a alteração na densidade para diversas degenerescências e so- 


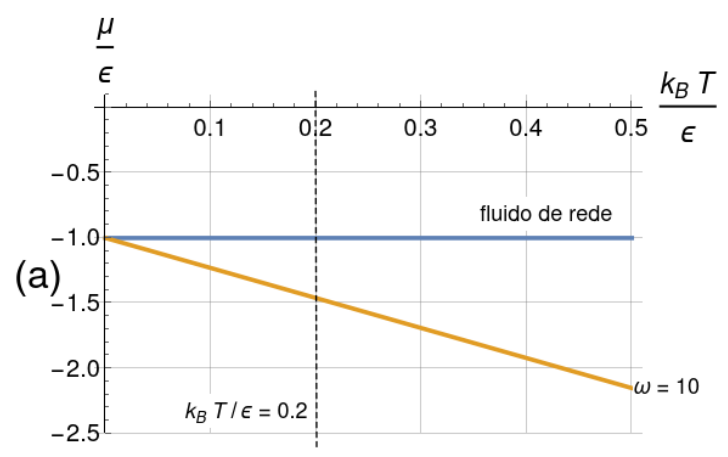

(b)

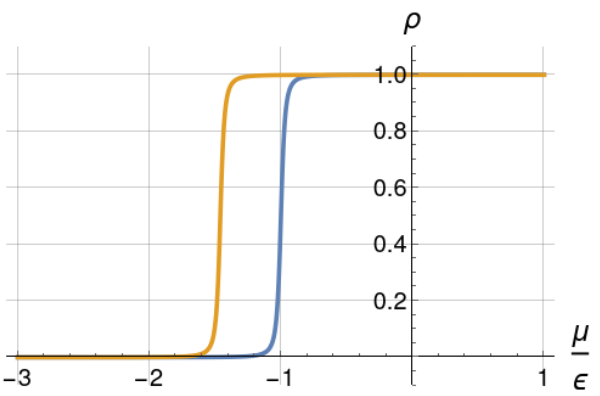

(d)

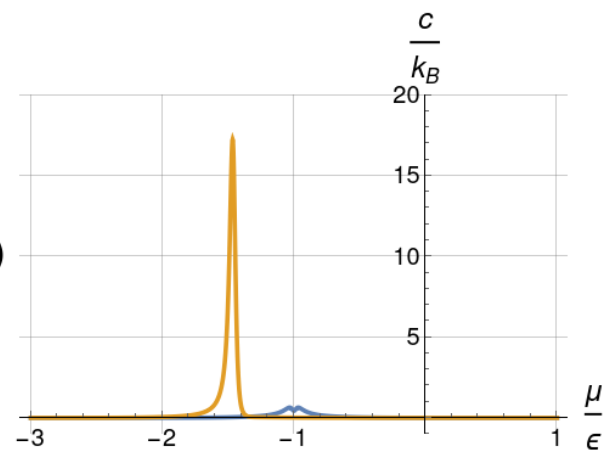

Figura 4.13: Em (a), o diagrama $\mu-T$ do fluido de rede, para o caso usual $(\omega=1)$ e o caso com degenerescência 10, a linha vertical tracejada, para $k_{B} T / \epsilon=0.2$, se refere à temperatura tomada fixa nos demais gráficos. O gráfico (b) mostra a densidade em função do potencial químico. O gráfico (c) mostra a entropia por sítio em função do potencial químico e o gráfico (d), o calor específico em função do potencial químico.

bre caminhos distintos. Em (b) cruzamos as linhas de pseudo-coexistência perpendicularmente, a uma distância de 0.5 do ponto $(0,-1)$ - da mesma maneira que fizemos para o modelo de Ising, através de uma família de retas (4.62). Novamente vemos que, para potencial químico fixo, a mudança é tão abrupta quanto maior é a degenerescência e, portanto, menor é a temperatura em que ocorre a pseudo-transição, gráfico (c). Por outro lado, no gráfico (d), para temperatura fixa, a taxa de mudança da densidade é visivelmente a mesma em todas as curvas, a menos de um deslocamento no valor de potencial químico, que coincide com o ponto onde as respectivas quase-fronteiras são atravessadas no diagrama (a). Lembramos que o mesmo ocorre para a magnetização no modelo de Ising degenerado, figura (4.5). 

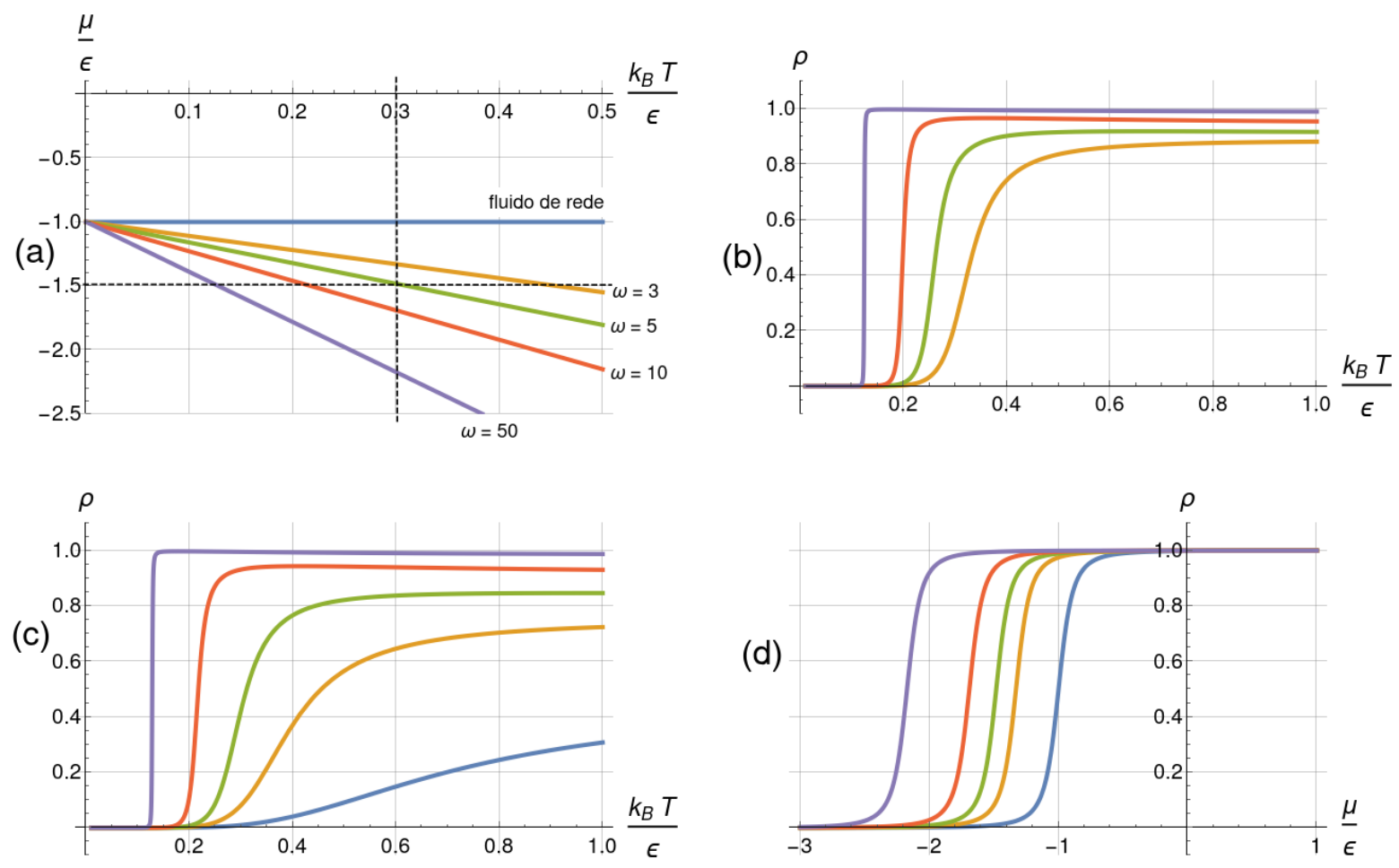

Figura 4.14: Em (a), o diagrama de quase-fases para o fluido de rede e para o fluido de rede degenerado $\operatorname{com} \omega=3,5,10$ e 50 . As cores das linhas são correspondentes às cores das curvas nos demais gráficos, isto é, as mesmas cores apresentam as mesmas degenerescências. As linhas tracejadas vertical e horizontal são a temperatura e o potencial químico fixos dos gráficos (d) e (c), respectivamente. O gráfico (b) é a densidade em função da temperatura ao atravessar as linhas de pseudo-coexistência perpendicularmente, à uma distância 0.5 do ponto $(0,-1)$. O gráfico (c) corresponde à densidade em função da temperatura para $\mu / \epsilon=-1.5$ fixo e o gráfico (d) é a densidade em função do potencial químico para $k_{B} T / \epsilon=0.3$ fixo. 


\section{Capítulo 5}

\section{O fluido de rede de Doniach (DLG)}

Recordamos que o modelo de Doniach, conquanto tente introduzir o conceito de área na monocamada, não define uma variação na densidade, pois cada sítio continua ocupado por uma única molécula. O fluido de rede de Doniach (DLG), proposto por Guidi e Henriques, é um modelo de três estados, onde o terceiro representa um sítio vazio na rede — na tese de doutorado de Guidi [8], o terceiro estado foi interpretado como vazio, já no artigo de Guidi e Henriques [9], foi considerado preenchido por água, de maneira a traduzir o efeito hidrofóbico nos sufactantes.

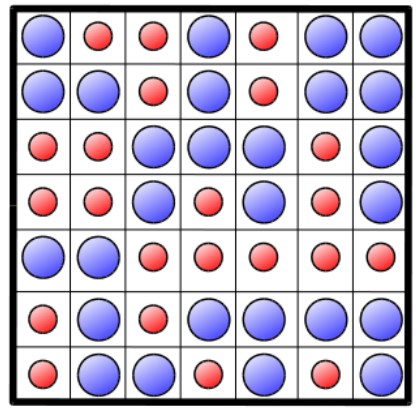

Doniach

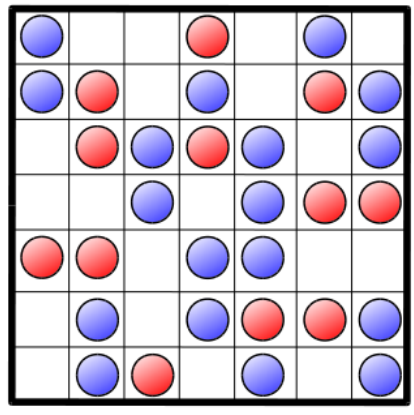

$D L G$

Figura 5.1: Representação do modelo de Doniach e do fluido de rede de Doniach (DLG), os círculos azuis e vermelhos se referem, respectivamente, ao estado desordenado e ordenado das caudas hidrofóbicas.

Considerando que a interação entre lipídios e buracos é nula, reescrevemos a Hamiltoniana (2.1) da maneira que segue

$$
\mathcal{H}(\{\eta\})=-\sum_{(i, j)} \delta_{i} \delta_{j}\left\{\epsilon_{o} \eta_{i} \eta_{j}+\epsilon_{d}\left(1-\eta_{i}\right)\left(1-\eta_{j}\right)+\epsilon_{o d}\left[\eta_{i}\left(1-\eta_{j}\right)+\left(1-\eta_{i}\right) \eta_{j}\right]\right\}
$$


onde $\delta_{i}$ vale 1 ou 0 para sítios ocupados e vazios ou preenchidos com moléculas de água, respectivamente.

É conveniente escrever a Hamiltoniana (5.1) usando uma única variável, para isso vamos evocar novamente o mapeamento (3.28) com a condição adicional de $\delta_{i}=\sigma_{i}^{2}$, onde agora temos $\sigma_{i}=-1,0$ e 1 , se referindo aos estados desordenado, vazio e ordenado, respectivamente, com o primeiro deles sendo $\omega$-degenerado, $\left(-1_{1},-1_{2}, \ldots,-1_{\omega}\right)$. Veja que construímos um modelo de Ising spin 1. A Hamiltoniana (5.1) se torna

$$
\mathcal{H}(\{\sigma\})=-J \sum_{(i, j)} \sigma_{i} \sigma_{j}-\Delta \sum_{(i, j)} \sigma_{i} \sigma_{j}\left(\sigma_{i}+\sigma_{j}\right)-K \sum_{(i, j)} \sigma_{i}^{2} \sigma_{j}^{2}
$$

onde

$$
J=\frac{\epsilon_{o}+\epsilon_{d}-2 \epsilon_{o d}}{4} \quad, \quad \Delta=\frac{\epsilon_{o}-\epsilon_{d}}{4} \quad \text { e } \quad K=\frac{\epsilon_{o}+\epsilon_{d}+2 \epsilon_{o d}}{4} .
$$

Estas novas energias de interação são todas positivas e refletem as seguintes propriedades: $J$ favorece a agregação de lipídios no mesmo estado, $\Delta$ governa a estabilidade do estado ordenado e $K$ favorece a agregação das moléculas, independente do estado.

Desta vez, transpomos a descrição do sistema para o ensemble grande canônico e dos campos $(T, L, \mu, H)$, tendo em vista que o número de partículas não é mais fixo. O motivo de querermos o campo como variável termodinâmica ficará evidente mais a frente. A energia livre nessas variáveis é obtida a partir da transformada de Legendre do grande potencial termodinâmico (4.66) com relação à variável $M$. Assim

$$
\Omega=\Phi-H M
$$

Diferenciando e usando a diferencial (4.67), considerando que haverá um termo adicional $+H d M$, obtemos

$$
d \Omega=-\sigma d L-S d T-N d \mu-M d H
$$

que nos dá as equações de estado 


$$
\begin{array}{cc}
\sigma=-\left(\frac{\partial \Omega}{\partial L}\right)_{T, \mu, H}, & S=-\left(\frac{\partial \Omega}{\partial T}\right)_{L, \mu, H}, \\
N=-\left(\frac{\partial \Omega}{\partial \mu}\right)_{T, L, H}, & M=-\left(\frac{\partial \Omega}{\partial H}\right)_{T, \mu, H} .
\end{array}
$$

A "Hamiltoniana"efetiva unidimensional construída a partir de (5.2), acrescida das contribuições da flutuação do número de partículas e da degenerescência

$$
\begin{array}{r}
\mathcal{H}_{e f}(\{\sigma\})=-\sum_{i=1}^{L}\left\{J \sigma_{i} \sigma_{i+1}+\Delta \sigma_{i} \sigma_{i+1}\left(\sigma_{i}+\sigma_{i+1}\right)+K \sigma_{i}^{2} \sigma_{i+1}^{2}+\frac{\mu}{2}\left(\sigma_{i}^{2}+\sigma_{i+1}^{2}\right)\right. \\
\left.+\frac{\ln \omega}{4 \beta}\left[\sigma_{i}\left(\sigma_{i}-1\right)+\sigma_{i+1}\left(\sigma_{i+1}-1\right)\right]\right\} .
\end{array}
$$

Ainda no idioma das variáveis de spin 1 , podemos definir uma densidade $\rho$, bem como uma "magnetização"por sítio, $m$ — daqui vem o motivo da nossa escolha de ensemble —, que nesse contexto traduz o grau de ordem do sistema: quanto mais próximo de 1, mais lipídios no estado ordenado, e quanto mais próximo de -1 , mais no estado desordenado. Densidade e "magnetização"são dadas, respectivamente, por

$$
\rho=\frac{N}{L}=\frac{1}{L} \sum_{i=1}^{L} \sigma^{2} \quad \text { e } \quad m=\frac{M}{L}=\frac{1}{L} \sum_{i=1}^{L} \sigma .
$$

Para obter $m$, vamos assumir por um instante que estamos tratando um sistema magnético e adicionar o termo linear

$$
-\frac{H}{2} \sum_{i=1}^{L}\left(\sigma_{i}+\sigma_{i+1}\right)
$$

à função (5.7). Assim que obtivermos as quantidades que nos interessam, fixamos $H=0$ e tudo volta ao normal.

Por uma questão de facilidade na notação das expressões, vamos definir os seguintes parâmetros 


$$
\left\{\begin{array}{l}
A:=e^{\beta(J-2 \Delta+K)}=e^{\beta \epsilon_{d}}, \\
B:=e^{\beta(J+2 \Delta+K)}=e^{\beta \epsilon_{o}} \\
C:=e^{\beta(-J+K)}=e^{\beta \epsilon_{o d}} \\
h:=e^{\beta H}
\end{array}\right.
$$

além da fugacidade $z=e^{\beta \mu}$. Por conseguinte, a matriz de transferência, é

$$
\mathbb{T}=\left[\begin{array}{ccc}
T(-1,-1) & T(-1,0) & T(-1,1) \\
T(0,-1) & T(0,0) & T(0,1) \\
T(1,-1) & T(1,0) & T(1,1)
\end{array}\right]=\left[\begin{array}{ccc}
\omega z h^{-1} A & \sqrt{\omega z h^{-1}} & \sqrt{\omega} z C \\
\sqrt{\omega z h^{-1}} & 1 & \sqrt{z h} \\
\sqrt{\omega} z C & \sqrt{z h} & z h B
\end{array}\right]
$$

Note que as entradas da matriz associadas à interação dos lipídios em determinados estados possuem um expoente no fator de Boltzmann dado pela energia de interação entre esses respectivos estados. Quer dizer, o elemento $T(-1,-1)$, associado à interação entre dois lipídios com caudas desordenadas, possui um expoente $\epsilon_{d}$, e para os outros fatores ocorre o mesmo.

A equação secular de (5.10) é dada por

$$
\lambda^{3}+a \lambda^{2}+b \lambda+c=0
$$

cujos coeficientes são

$$
\left\{\begin{array}{l}
a=-\left[1+z\left(h^{-1} \omega A+h B\right)\right] \\
b=z\left[\left(h^{-1} \omega A+h B\right)+\omega z\left(A B-C^{2}\right)-\left(h^{-1} \omega+h\right)\right] \\
c=\omega z^{2}\left[(A+B-2 C)-\left(A B-C^{2}\right)\right]
\end{array}\right.
$$

ou, mais explicitamente 


$$
\left\{\begin{array}{l}
a=-\left[1+e^{\beta\left(\mu+\epsilon_{o}-H\right)}+e^{\beta\left(\mu+\epsilon_{d}+H\right)}\right] \\
b=\omega e^{\beta\left(\mu+\epsilon_{o}-H\right)}+e^{\beta\left(\mu+\epsilon_{d}-H\right)}+\omega e^{\beta\left(2 \mu+\epsilon_{o}+\epsilon_{d}\right)}-\omega e^{2 \beta\left(\mu+\epsilon_{o d}\right)}-\omega e^{-\beta(H+\mu)}-e^{\beta(H+\mu)}, \\
c=\omega\left[e^{\beta\left(2 \mu+\epsilon_{o}\right)}+e^{\beta\left(2 \mu+\epsilon_{d}\right)}-2 e^{\beta\left(2 \mu+\epsilon_{o d}\right)}-e^{\beta\left(2 \mu+\epsilon_{o}+\epsilon_{d}\right)}+e^{2 \beta\left(\mu+\epsilon_{o d}\right)}\right] .
\end{array}\right.
$$

Sendo a equação (5.11) de grau 3, a sua resolução é um pouco trabalhosa e precisa ser feita com cuidado. No Apêndice B apresentamos uma demonstração para obtenção das raízes, que são dadas por

$$
\lambda_{k}=-\frac{a}{3}+\frac{2 \sqrt{\Delta_{0}}}{3} \cos \left(\frac{2 k \pi+\theta}{3}\right), \quad k=0,1,2,
$$

onde

$$
\theta=\tan ^{-1}\left(\frac{\sqrt{\Delta}}{\Delta_{1}}\right)
$$

e

$$
\left\{\begin{array}{l}
\Delta_{0}=a^{2}-3 b \\
\Delta_{1}=-2 a^{3}+9 a b-27 c \\
\Delta=4 \Delta_{0}^{3}-\Delta_{1}^{2}=27\left(a^{2} b^{2}-4 b^{3}-4 a^{3} c+18 a b c-27 c^{2}\right)
\end{array}\right.
$$

$\mathrm{O}$ discriminante $\Delta$ tem uma propriedade similar à que tem no caso de uma equação do $2^{\circ}$ grau: seu sinal indica a existência de raízes reais ou complexas, quando $\Delta>0$, todas as raízes da equação são reais - alertamos para não confundir o $\Delta$ do discriminante da equação cúbica com aquele da energia de interação da Hamiltoniana (5.7).

Dada a complexidade da expressão, verificamos o sinal de $\Delta$ através de uma varredura numérica, para diversos valores de $\omega$ e $\mu$, mostramos alguns resultados na figura (5.2). Para o conjunto de parâmetros que adotamos na análise do sistema, $\Delta / J=0.4 \mathrm{e} K / J=1$, a condição de realidade das raízes (5.14) sempre se satisfaz para temperatura finita.

Esse mesmo método é utilizado na verificação do maior autovalor que, como mostra a figura 

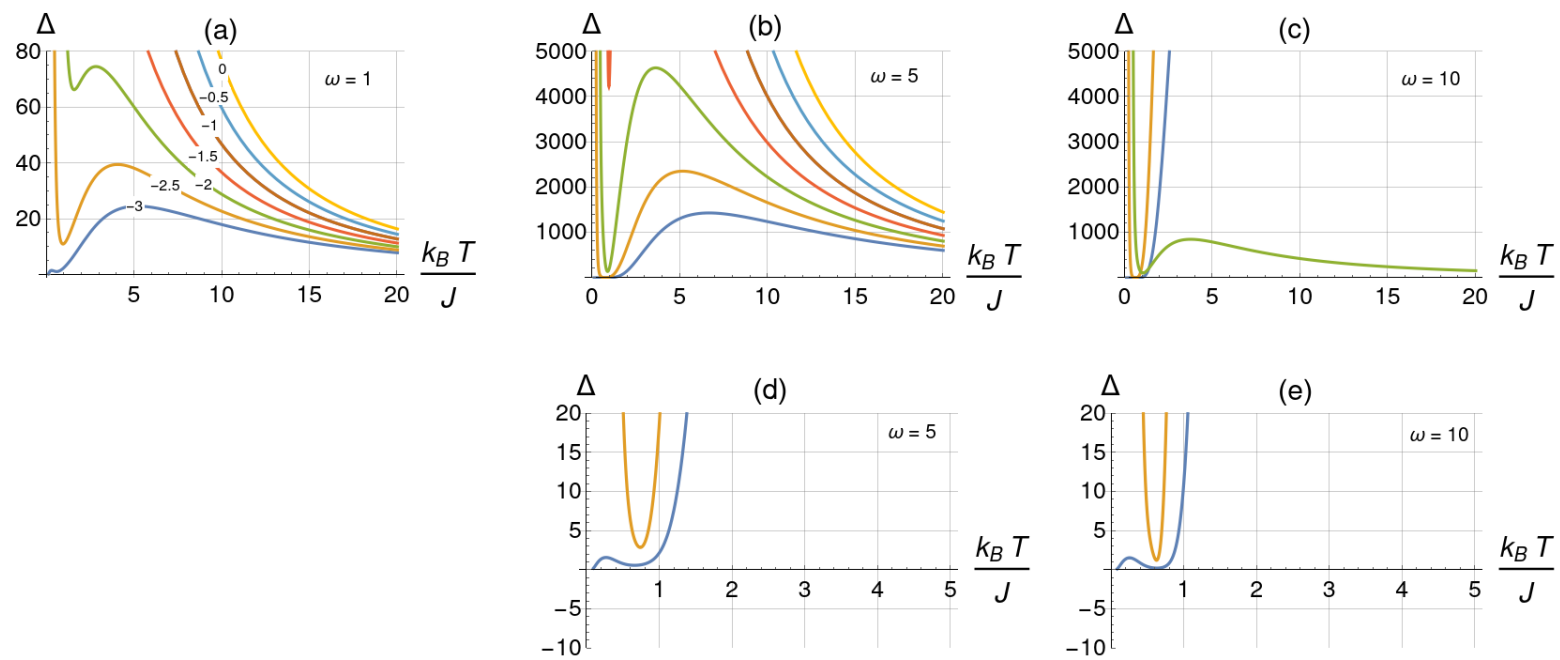

Figura 5.2: Gráficos do discriminante $\Delta$ da equação cúbica em função da temperatura reduzida. Todos se referem a valores de potencial químico $\mu / J=-3,-2.5,-2,-1.5,-1,-0.5$ e 0 . Nas curvas, à medida que o $\mu$ aumenta, $\Delta$ também fica maior, e as cores são correspondentes em todos os gráficos, isto é, as mesmas cores possuem o mesmo $\mu$. Os gráficos (a), (b) e (c) se referem às degenerescências $\omega=1,2$ e 3 , respectivamente. Os gráficos (d) e (e) são os mesmos parâmetros que os usados em (b) e (c), respectivamente, mas mostrando uma região mais próxima à origem. Os coeficientes da Hamiltoniana considerados aqui são $\Delta / J=0.4 \mathrm{e} K / J=1$.

(5.3), é o que corresponde ao autovalor $\lambda_{0}$.

Destarte, no limite termodinâmico, a grande função de partição será

$$
\mathcal{Z}=\lambda_{0}^{L}+\lambda_{1}^{L}+\lambda_{2}^{L}=\lambda_{0}^{L}\left[1+\left(\frac{\lambda_{1}}{\lambda_{0}}\right)^{L}+\left(\frac{\lambda_{2}}{\lambda_{0}}\right)^{L}\right] \stackrel{L \rightarrow \infty}{\longrightarrow} \lambda_{0}^{L} .
$$

Explicitamente

$$
\mathcal{Z}(T, \mu, L, H ; \omega)=-\left\{\frac{1}{3}\left[a-2 \sqrt{\Delta_{0}} \cos \left(\frac{\theta}{3}\right)\right]\right\}^{L}
$$

A energia livre é

$$
\Omega(T, \mu, L, H ; \omega)=-L k_{B} T \ln \left\{-\frac{1}{3}\left[a-2 \sqrt{\Delta_{0}} \cos \left(\frac{\theta}{3}\right)\right]\right\} .
$$

A magnetização e a entropia por sítio podem ser obtidas das equações de estado (5.6), a densidade, a energia interna por sítio e a capacidade térmica por sítio à potencial químico constante, através de equações análogas às obtidas para o fluido de rede, (4.78), (4.79) e (4.92), respectivamente, levando em consideração as variáveis mantidas fixas.

Devido ao tamanho das expressões, é conveniente operarmos por etapas. Seguem as deriva- 

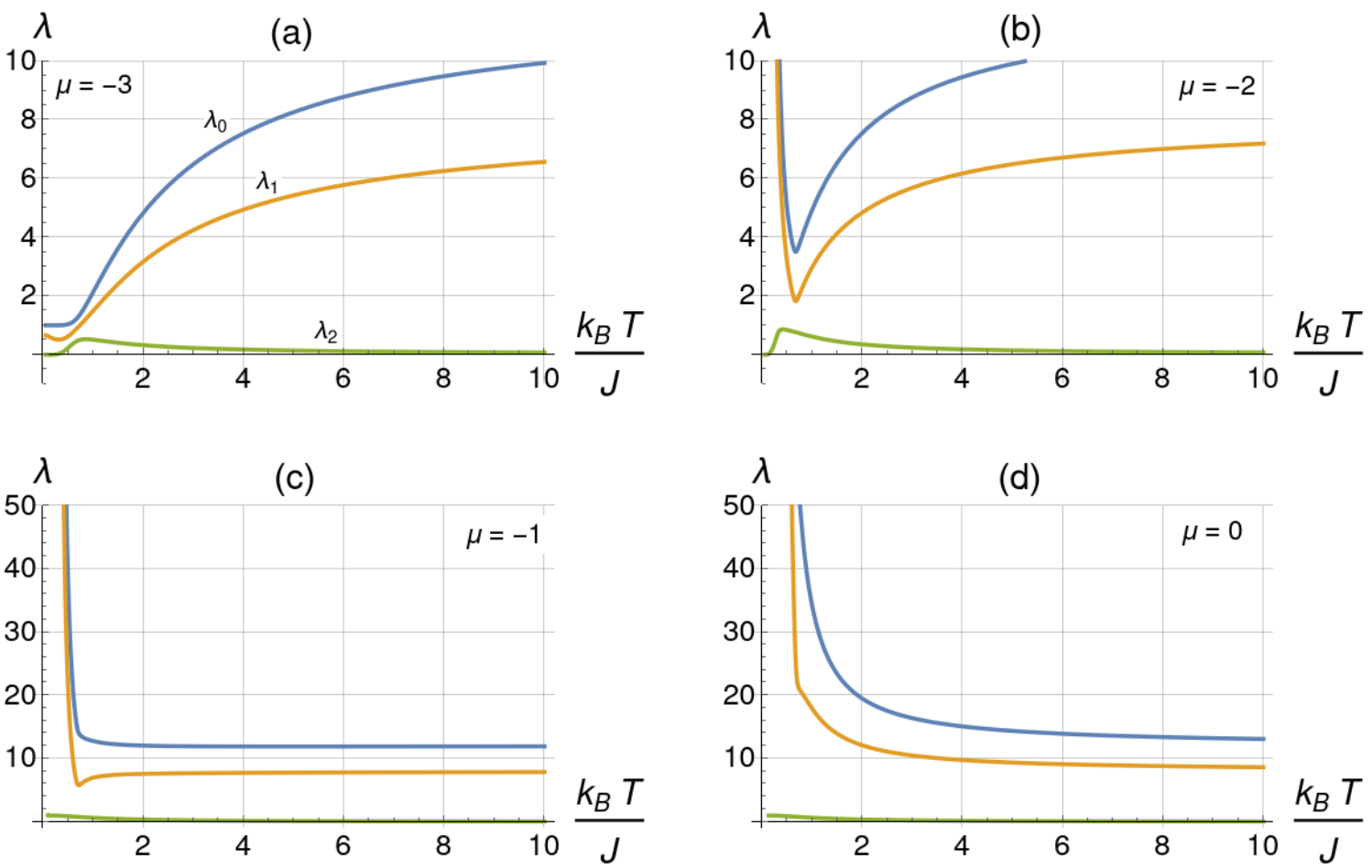

Figura 5.3: Os gráficos representam os autovalores associados à matriz de transferência do DLG unidimensional em função da temperatura. As cores das curvas em todos os gráficos são correspondentes, sendo azul, laranja e verde para $\lambda_{0}, \lambda_{1}$ e $\lambda_{2}$, respectivamente. Em (a), (b), (c) e (d) os potenciais químicos foram fixados em, respectivamente, $\mu / J=-3,-2,-1$ e 0 .

das com relação a uma das variáveis $x=\beta, \mu, H$, enquanto as outras são mantidas fixas

$$
\begin{gathered}
\frac{\partial \lambda_{0}}{\partial x}=-\frac{1}{3} \frac{\partial a}{\partial x}+\frac{2}{3}\left[\frac{1}{2 \sqrt{\Delta_{0}}} \frac{\partial \Delta_{0}}{\partial x} \cos \left(\frac{\theta}{2}\right)-\frac{\sqrt{\Delta_{0}}}{3} \frac{\partial \theta}{\partial x} \sin \left(\frac{\theta}{3}\right)\right], \\
\frac{\partial^{2} \lambda_{0}}{\partial x^{2}}=-\frac{1}{3} \frac{\partial^{2} a}{\partial x^{2}}+\frac{1}{54 \Delta_{0}^{\frac{3}{2}}}\left[18 \Delta_{0} \frac{\partial^{2} \Delta_{0}}{\partial x^{2}}-9\left(\frac{\partial \Delta_{0}}{\partial x}\right)^{2}-\left(2 \frac{\partial \theta}{\partial x} \Delta_{0}\right)^{2} \cos \left(\frac{\theta}{3}\right)\right] \\
-\frac{1}{9 \sqrt{\Delta_{0}}}\left(\frac{\partial \Delta_{0}}{\partial x} \frac{\partial \theta}{\partial x}+\frac{\partial^{2} \theta}{\partial x^{2}} \sqrt{\Delta_{0}}\right) \sin \left(\frac{\theta}{3}\right), \\
\frac{\partial \theta}{\partial x}=\frac{1}{2 \sqrt{\Delta}\left(\Delta+\Delta_{1}^{2}\right)}\left(\Delta_{1} \frac{\partial \Delta}{\partial x}-2 \Delta \frac{\partial \Delta_{1}}{\partial x}\right),
\end{gathered}
$$




$$
\begin{aligned}
& \frac{\partial^{2} \theta}{\partial x^{2}}=\frac{1}{2 \sqrt{\Delta}\left(\Delta+\Delta_{1}^{2}\right)^{2}}\left\{\left(\Delta+\Delta_{1}^{2}\right)\left(\Delta_{1} \frac{\partial^{2} \Delta}{\partial x^{2}}-2 \frac{\partial^{2} \Delta_{1}}{\partial x^{2}} \Delta-\frac{\partial \Delta_{1}}{\partial x} \frac{\partial \Delta}{\partial x}\right)\right. \\
& \left.-\left[\frac{\partial \Delta}{\partial x} \frac{\left(\Delta+\Delta_{1}^{2}\right)}{2 \Delta}+\frac{\partial \Delta}{\partial x}+2 \Delta_{1} \frac{\partial \Delta_{1}}{\partial x}\right]\left(\Delta_{1} \frac{\partial \Delta}{\partial x}-2 \frac{\partial \Delta_{1}}{\partial x} \Delta\right)\right\} \\
& \frac{\partial \Delta_{0}}{\partial x}=2 a \frac{\partial a}{\partial x}-3 \frac{\partial b}{\partial x}, \\
& \frac{\partial^{2} \Delta_{0}}{\partial x^{2}}=2\left[\left(\frac{\partial a}{\partial x}\right)^{2}+a \frac{\partial^{2} a}{\partial x^{2}}\right]-3 \frac{\partial^{2} b}{\partial x^{2}} \\
& \frac{\partial \Delta_{1}}{\partial x}=-6 a^{2} \frac{\partial a}{\partial x}+9\left(\frac{\partial a}{\partial x} b+a \frac{\partial b}{\partial x}\right)-27 \frac{\partial c}{\partial x}, \\
& \frac{\partial^{2} \Delta_{1}}{\partial x^{2}}=-6\left[2 a\left(\frac{\partial a}{\partial x}\right)^{2}+a^{2} \frac{\partial^{2} a}{\partial x^{2}}\right]+9\left(\frac{\partial^{2} a}{\partial x^{2}} b+a \frac{\partial^{2} b}{\partial x^{2}}+2 \frac{\partial a}{\partial x} \frac{\partial b}{\partial x}\right)-27 \frac{\partial^{2} c}{\partial x^{2}} \\
& \frac{\partial \Delta}{\partial x}=12 \Delta_{0}^{2} \frac{\partial \Delta_{0}}{\partial x}-2 \Delta_{1} \frac{\partial \Delta 1}{\partial x} \\
& \frac{\partial^{2} \Delta}{\partial x^{2}}=12\left[2 \Delta_{0}\left(\frac{\partial \Delta_{0}}{\partial x}\right)^{2}+\Delta_{0}^{2} \frac{\partial^{2} \Delta_{0}}{\partial x^{2}}\right]-2\left[\left(\frac{\partial \Delta_{1}}{\partial x}\right)^{2}+\Delta_{1} \frac{\partial^{2} \Delta_{1}}{\partial x^{2}}\right] \\
& \left\{\begin{array}{l}
\frac{\partial a}{\partial \mu}=-\beta z(\omega A+B) \\
\frac{\partial b}{\partial \mu}=\beta\left[b+\omega z^{2}\left(A B-C^{2}\right)\right] \\
\frac{\partial c}{\partial \mu}=2 \beta c
\end{array}\right. \\
& \left\{\begin{array}{l}
\frac{\partial a}{\partial H}=\beta z(\omega A-B) \\
\frac{\partial b}{\partial H}=\beta z(B-\omega A+\omega-1) \\
\frac{\partial c}{\partial H}=0
\end{array}\right.
\end{aligned}
$$




$$
\left\{\begin{array}{l}
\frac{\partial a}{\partial \beta}=-z\left[\omega\left(\mu+\epsilon_{d}\right) A+\left(\mu+\epsilon_{o}\right) B\right], \\
\frac{\partial b}{\partial \beta}=z\left\{\left(\mu+\epsilon_{d}\right) \omega A+\left(\mu+\epsilon_{o}\right) B+\omega z\left[\left(2 \mu+\epsilon_{d}+\epsilon_{o}\right) A B-2\left(\mu+\epsilon_{o d}\right) C^{2}\right]-\mu(\omega+1)\right\}, \\
\frac{\partial c}{\partial \beta}=\omega z^{2}\left\{\left(2 \mu+\epsilon_{d}\right) A+\left(2 \mu+\epsilon_{o}\right) B-2\left(2 \mu+\epsilon_{o d}\right) C-\left(2 \mu+\epsilon_{d} \epsilon_{o}\right) A B+2\left(\mu+\epsilon_{o d}\right) C^{2}\right\}
\end{array}\right.
$$

$$
\left\{\begin{array}{l}
\frac{\partial^{2} a}{\partial \beta^{2}}=-z\left[\omega\left(\mu+\epsilon_{d}\right)^{2} A+\left(\mu+\epsilon_{o}\right)^{2} B\right], \\
\frac{\partial^{2} b}{\partial \beta^{2}}=z\left\{\left(\mu+\epsilon_{d}\right)^{2} \omega A+\left(\mu+\epsilon_{o}\right)^{2} B+\omega z\left[\left(2 \mu+\epsilon_{d}+\epsilon_{o}\right)^{2} A B-4\left(\mu+\epsilon_{o d}\right)^{2} C^{2}\right]-\mu^{2}(\omega+1)\right\}, \\
\frac{\partial^{2} c}{\partial \beta^{2}}=\omega z^{2}\left\{\left(2 \mu+\epsilon_{d}\right)^{2} A+\left(2 \mu+\epsilon_{o}\right)^{2} B-2\left(2 \mu+\epsilon_{o d}\right)^{2} C-\left(2 \mu+\epsilon_{d} \epsilon_{o}\right)^{2} A B+4\left(\mu+\epsilon_{o d}\right)^{2} C^{2}\right\} .
\end{array}\right.
$$

Note que já escrevemos as derivadas de $a, b$ e $c$ tomando $H=0$.

\section{As funções termodinâmicas}

Finalmente, vamos escrever as grandezas em termos das derivadas obtidas, agora omitindo a dependência com $H$, já que a sua função já foi cumprida.

A densidade

$$
\rho(T, \mu ; \omega)=-\frac{k_{B} T}{\lambda_{0}}\left\{\frac{1}{3} \frac{\partial a}{\partial \mu}-\frac{2}{3}\left[\frac{1}{2 \sqrt{\Delta_{0}}} \frac{\partial \Delta_{0}}{\partial \mu} \cos \left(\frac{\theta}{2}\right)-\frac{\sqrt{\Delta_{0}}}{3} \frac{\partial \theta}{\partial \mu} \sin \left(\frac{\theta}{3}\right)\right]\right\} .
$$

A "magnetização"por sítio

$$
m(T, \mu ; \omega)=-\frac{k_{B} T}{\lambda_{0}}\left\{\frac{1}{3} \frac{\partial a}{\partial H}-\frac{2}{3}\left[\frac{1}{2 \sqrt{\Delta_{0}}} \frac{\partial \Delta_{0}}{\partial H} \cos \left(\frac{\theta}{2}\right)-\frac{\sqrt{\Delta_{0}}}{3} \frac{\partial \theta}{\partial H} \sin \left(\frac{\theta}{3}\right)\right]\right\}
$$

A energia interna por sítio 


$$
u(T, \mu ; \omega)=\mu \rho(T, \mu ; \omega)-\frac{1}{\lambda_{0}}\left(\frac{\partial \lambda_{0}}{\partial \beta}\right)_{\mu}
$$

A entropia por sítio

$$
s(T, \mu ; \omega)=k_{B} \ln \lambda_{0}+\frac{1}{T} \frac{1}{\lambda_{0}}\left\{\frac{1}{3} \frac{\partial a}{\partial \beta}-\frac{2}{3}\left[\frac{1}{2 \sqrt{\Delta_{0}}} \frac{\partial \Delta_{0}}{\partial \beta} \cos \left(\frac{\theta}{2}\right)-\frac{\sqrt{\Delta_{0}}}{3} \frac{\partial \theta}{\partial \beta} \sin \left(\frac{\theta}{3}\right)\right]\right\} .
$$

A capacidade térmica por sítio a potencial químico constante

$$
c_{\mu}(T ; \omega)=k_{B} \beta^{2}\left[\frac{1}{\lambda_{0}}\left(\frac{\partial^{2} \lambda_{0}}{\partial \beta^{2}}\right)_{\mu}-u^{2}(T, \mu ; \omega)\right]
$$

\section{As quase-fases}

No fluido de rede de Doniach também observamos o fenômeno das pseudo-transições. No diagrama $\mu-T$, figura (5.4), distinguimos três regiões com características próprias distintas, separadas por fronteiras mais ou menos bem definidas: as quase-fases fluido ordenado, fluido desordenado e gás — as duas primeiras sendo análogas às fases que mencionamos no capítulo 2: gel e fluido, respectivamente, e a terceira, uma fase caracterizada pela baixa densidade de lipídios. Embora seja difícil verificar analiticamente a competição entre os termos da diagonal principal a partir das funções termodinâmicas — como foi possível fazer no modelo de Ising —, no DLG essa característica também se apresenta, como será possível ver nos resultados numéricos. As entradas da diagonal da matriz (5.10) ditam o comportamento da energia livre, através de uma competição que se torna tão acentuada quanto menor for a temperatura do sistema. Cada fator é dominante na sua respectiva quase-fase: $T(-1,-1)$ para o fluido desordenado, $T(1,1)$ para o fluido ordenado e $T(0,0)$ para o gás. Também podemos obter uma expressão para a quase-fronteira a partir da coincidência entre esses termos.

A pseudo-transição entre os estados gás e líquido desordenado é obtida de $T(-1,-1)=$ $T(0,0)$

$$
T=-\frac{\mu+\epsilon_{d}}{k_{B} \ln \omega}
$$


Observe que essa relação é idêntica à do fluido de rede degenerado (4.93).

A pseudo-transição entre os estados líquido desordenado e líquido ordenado é calculada a partir de $T(-1,-1)=T(1,1)$, que nos fornece

$$
T=\frac{\epsilon_{o}-\epsilon_{d}}{k_{B} \ln \omega}
$$

que é um valor fixo de temperatura, dependente da energia de interação entre os lipídios e da degenerescência.

Por fim, a pseudo-transição entre os estados gás e líquido ordenado pode ser obtida de $T(0,0)=T(1,1)$, de maneira que

$$
\frac{\mu+\epsilon_{o}}{k_{B} T}=0
$$

que, para temperatura finita, só se verifica quando $\mu=-\epsilon_{o}$.

O diagrama de quase-fases para o sistema com degenerescência 10 é mostrado juntamente com um diagrama de densidade de entropia na figura (5.4). Utilizamos para a análise os valores $\Delta / J=0.4$ e $K / J=1$, que correspondem às energias de interação $\epsilon_{o} / J=2.8, \epsilon_{d} / J=1.2 \mathrm{e}$ $\epsilon_{o d} / J=0$, segundo as relações (5.3). Assim, para um sistema cuja degenerescência no estado -1 vale 10 , temos as seguintes linhas de pseudo-coexistência

$$
\begin{cases}k_{B} T / J=-0.43 \mu-0.52 & \text { [gás - líquido desordenado] }, \\ \mu / J=-2.8 & \text { [líquido ordenado - líquido desordenado] } \\ k_{B} T / J=0.69 & \text { [gás - líquido ordenado] } .\end{cases}
$$

Podemos confrontar o diagrama construído a partir dos elementos da diagonal principal (5.41) com o diagrama de densidade da entropia por sítio, ambos na figura (5.4) e ainda com diagrama obtido por Guidi [8] através da aproximação de campo médio, figura (5.5). Vemos que todos apresentam regiões similares associadas às fases termodinâmicas gás, líquido ordenado e líquido desordenado, com fronteiras qualitativamente iguais, excetuando as vizinhanças do "ponto triplo". No diagrama da densidade de entropia, vemos que a quase-fronteira, linha 
horizontal, entre as quase-fases líquido ordenado e desordenado apresenta uma queda ao diminuirmos o potencial químico, em aproximadamente $\mu / J \approx-2$. A linha diagonal que separa as quase-fases gás e líquido desordenado encontra as outras duas um pouco abaixo da pseudotransição horizontal. Além disso, ela não é tão nítida quanto as outras duas, sugerindo que a pseudo-transição entre essas regiões é bem menos abrupta, e a linha se torna mais dispersa conforme a temperatura do sistema aumenta.

Evidentemente, essas informações se perdem ao traduzirmos as quase-fronteiras nas equações (5.41), obtidas através da coincidência dos elementos de matriz da diagonal principal. Não obstante, essas expressões ainda descrevem razoavelmente bem as linhas de pseudo-transição vertical e horizontal, com precisão tanto melhor quanto menor é a temperatura.

Vemos também que no resultado obtido por Guidi, figura (5.5), a vizinhança do "ponto triplo", se comporta de modo diferente da nossa, a pseudo-transição entre as quase-fases gás e líquido desordenado encontra as outras duas mais acima do que o resultado que obtemos, em um valor de $\mu$ um pouco maior, antes da linha horizontal começar a decair.
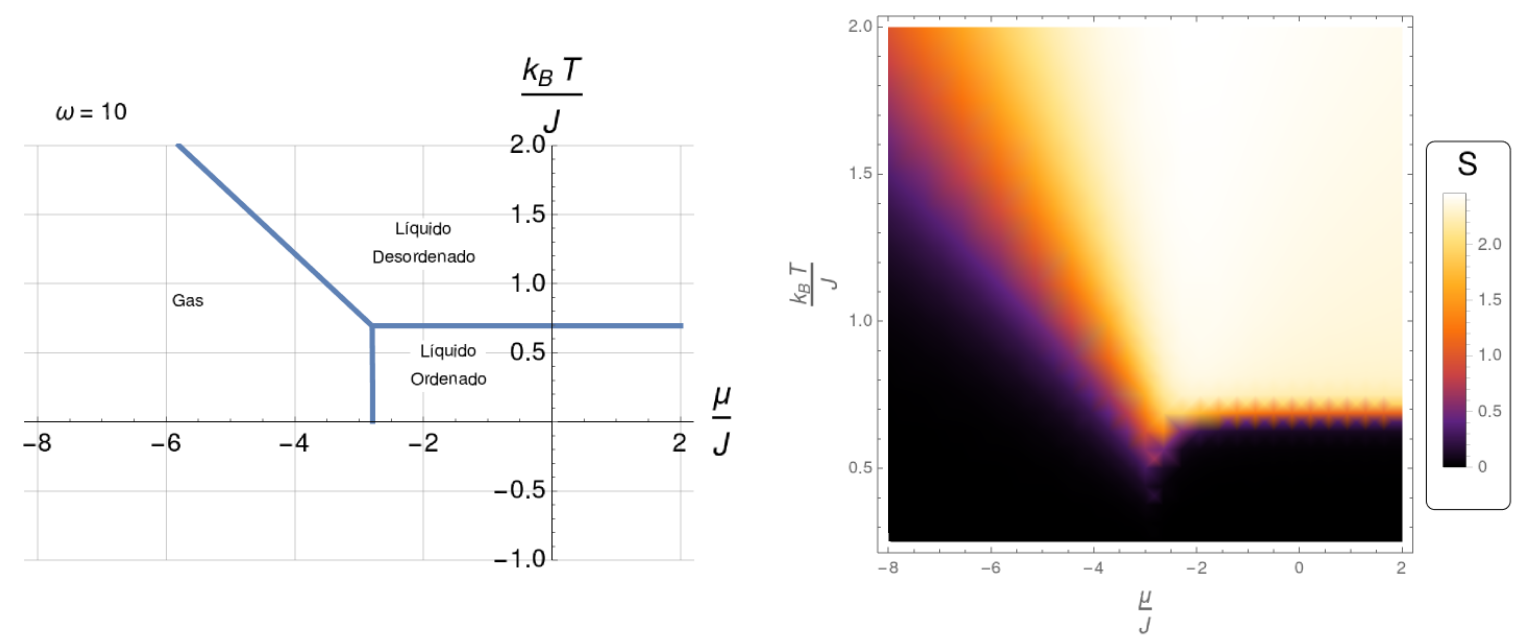

Figura 5.4: À esquerda, as linhas obtidas através da igualdade entre os elementos da diagonal principal da matriz de transferência do modelo, para degenerescência 10 no estado desordenado. À direita, um diagrama de densidade da entropia por sítio, do mesmo modelo, cujos eixos são correspondentes aos do gráfico da esquerda. Para ambos, os parâmetros usados são $\Delta / J=0.4$ e $K / J=1$.

O diagrama (5.6) mostra o comportamento das quase-fases sob variação da degenerescência. Duas consequências merecem sobressair:

Por um lado, o aumento da degenerescência provoca uma aproximação das quase-fronteiras da região de baixas temperaturas, o que implica, como vimos nos modelos de dois estados, 


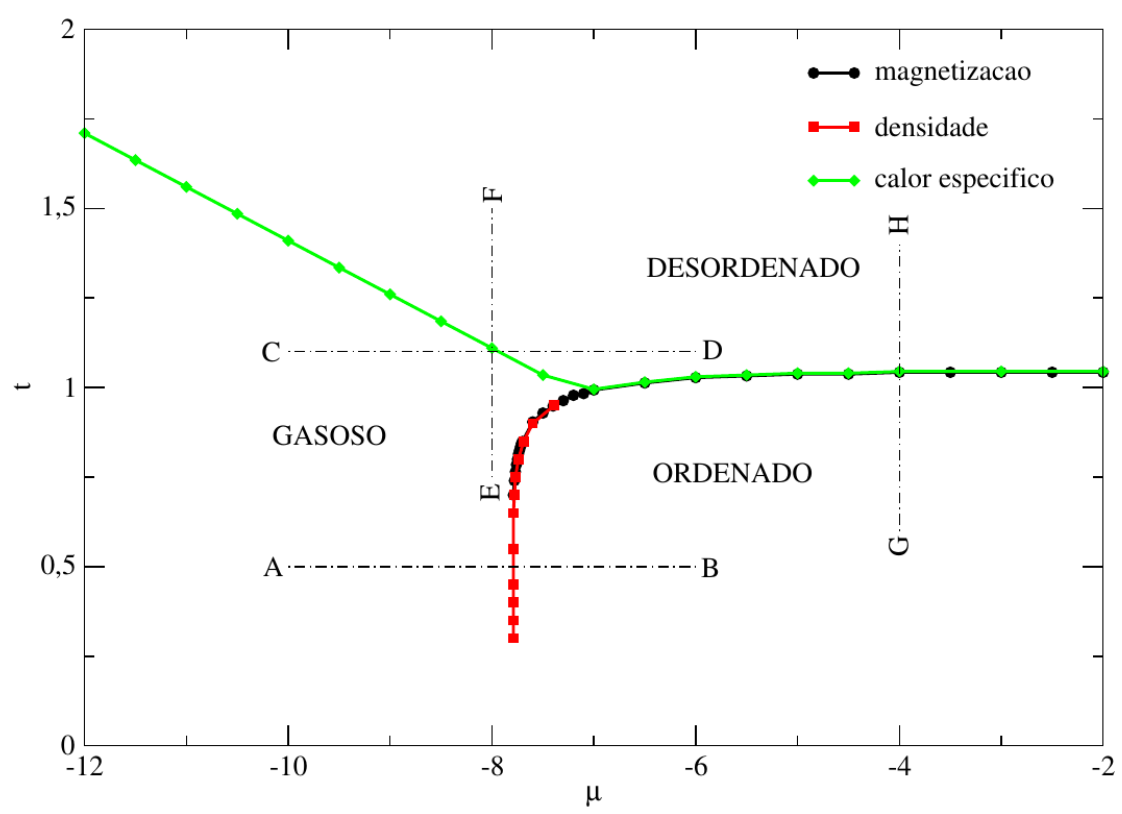

Figura 5.5: Diagrama de fases do fluido de rede de Doniach, obtido através da aproximação de campo médio, retirado da tese de doutorado de Henrique Guidi [8].

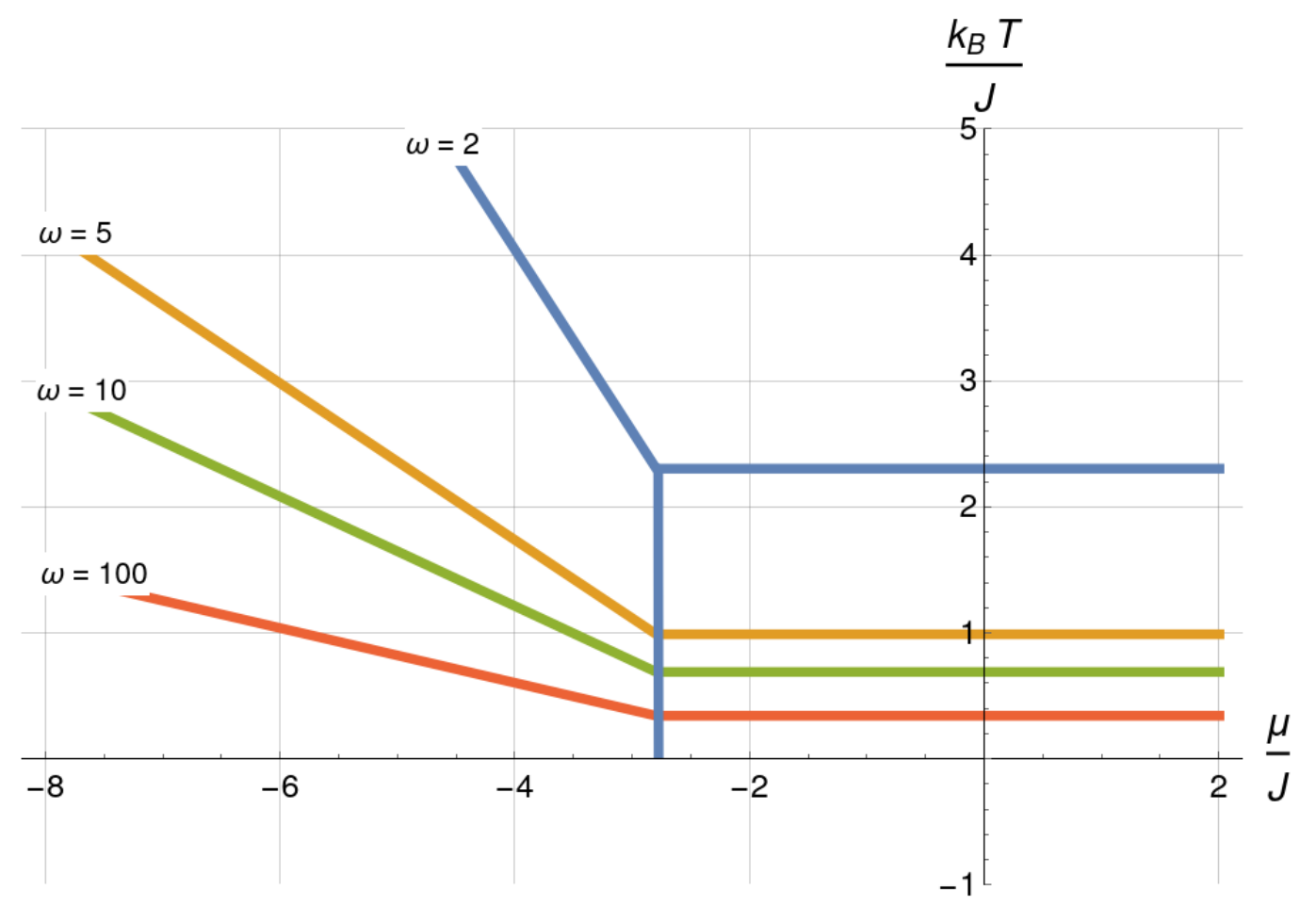

Figura 5.6: Diagrama de quase-fases do fluido de rede de Doniach unidimensional para as degenerescências $2,5,10$ e 100 no estado desordenado (-1), obtido a partir da igualdade entre os elementos da diagonal principal na matriz de transferência. 
em aumento do contraste entre essas regiões, causando mudanças mais abruptas na pseudotransição — além disso, os termos fora da diagonal principal na matriz (5.10) tendem a zero ao resfriar o sistema sempre que $\mu<0$, satisfazendo a condição proposta por Rojas [20], que exige que $T(a, b \neq a) \ll 1$ para o surgimento de pseudo-transições. Por outro lado, também vemos que o aumento de $\omega$ diminui a área das regiões associadas às quase-fases gás e líquido ordenado, mostrando um favorecimento à quase-fase líquido-desordenado, o que é razoável, dado que a degenerescência é nos estados desordenados, privilegiando, por conseguinte, a agregação de lipídios nessa configuração.

O caso $\omega=1$ - similar ao modelo BEG [43] —, precisa de um olhar mais cuidadoso. As expressões que descrevem as quase-fronteiras gás-fluido desordenado (5.38) e gás-fluido ordenado (5.39), indicam que essa condição implica em divergências na temperatura, sugerindo a ausência dessas linhas. A exceção é a pseudo-transição gás-fluido ordenado (5.40), que fornece $\mu=-\epsilon_{o}$. A figura (5.7) mostra os gráficos de densidade da entropia por sítio e da "magnetização"por sítio, para o sistema não-degenerado. Com efeito, observamos apenas uma linha, em $\mu / J=-2.8$ para temperaturas baixas, que separa duas regiões com propriedades nitidamente distintas, uma de "magnetização"1 e a outra com 0 , ambas com entropia nula, a região de magnetização negativa deixa de aparecer no diagrama. Por fim, é interessante notar que o diagrama de densidade de magnetização se assemelha muito ao diagrama de fases do modelo de Blume-Capel [44, 45], deixamos esse caso como uma investigação para o futuro.
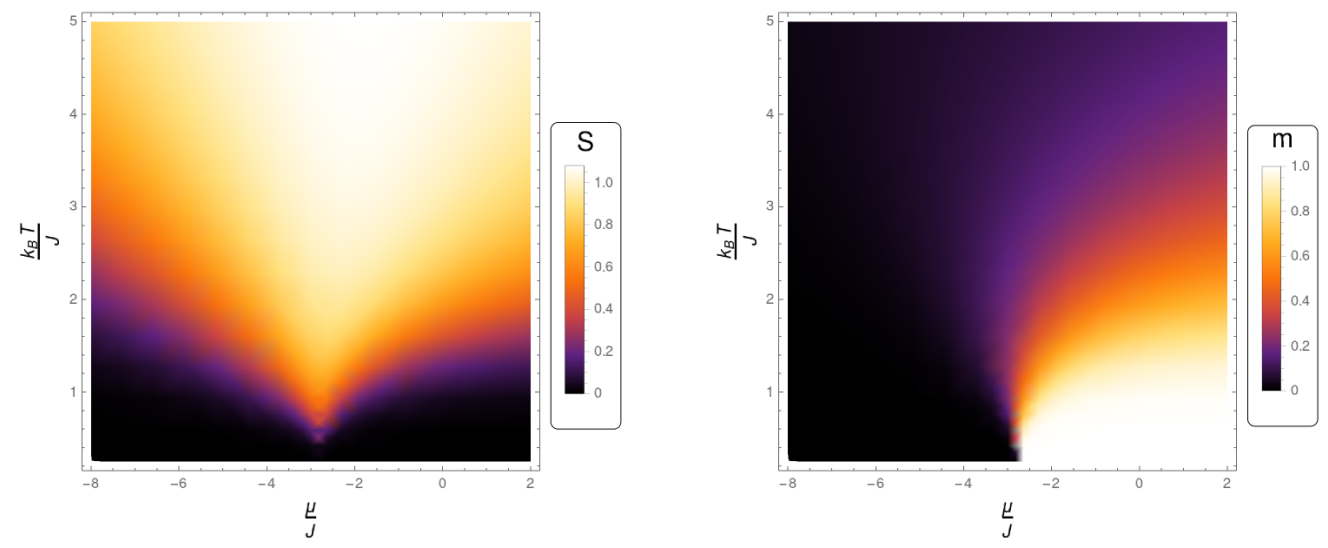

Figura 5.7: À esquerda, diagrama $\mu-T$ de densidade de entropia por sítio, a região mais escura remonta à entropia nula, enquanto as mais claras são para entropia $s / k_{B}=\ln 2$. À direita, a densidade de "magnetização"por sítio, cujos eixos coincidem com os eixos da entropia, onde as regiões mais escuras se referem à magnetização nula enquanto a mais claras, à magnetização nula. 


\section{Resultados numéricos}

Os gráficos de algumas das grandezas calculadas (5.33) - (5.37), são exibidos nas figuras que seguem.

(a)

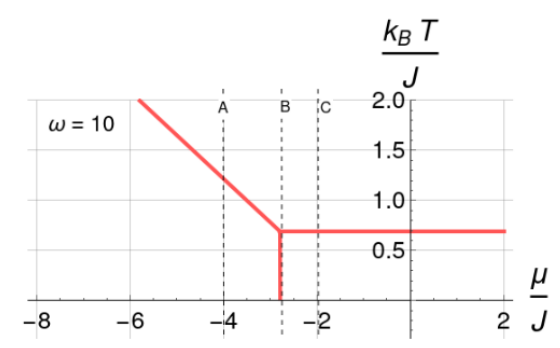

(c)

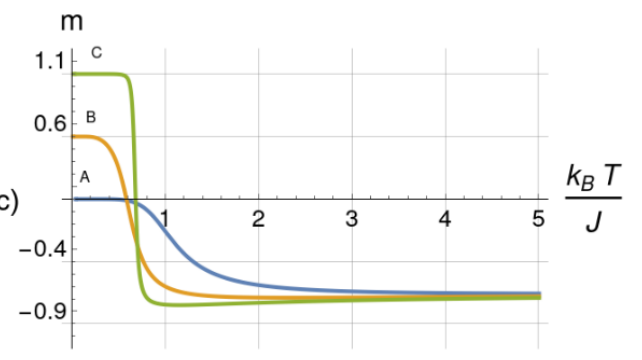

(b)

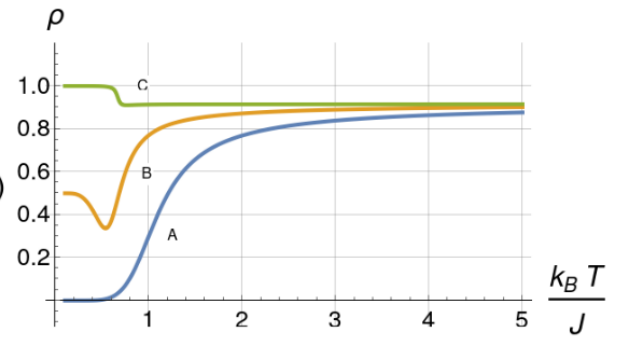

(d)

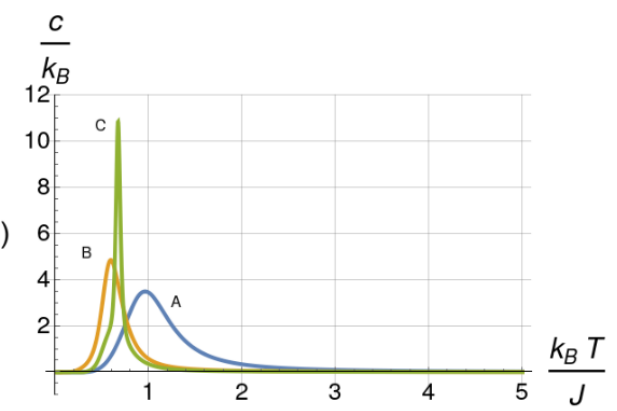

Figura 5.8: Em (a), o diagrama de quase-fases do DLG para degenerescência 10, as linhas verticais tracejadas $A, B$ e $C$ são os valores de potencial químico fixos $\mu / J=-4,-2.8$ e -2 e coincidem com as curvas dos demais gráficos, legendadas com as mesmas letras. Em (b), a densidade em função da temperatura. Em (c), a "magnetização"por sítio em função da temperatura. Em (d), a capacidade térmica por sítio a potencial químico constante em função da temperatura. Todos os gráficos são para $\omega=10$.

Na figura (5.8), buscamos fixar valores de $\mu$ de sorte a cruzar pontos de interesse no diagrama de quase-fases, gráfico (a). A curva $A$ tem $\mu / J=-4$, a curva $B$ foi escolhida de modo a satisfazer (5.40), sendo $\mu / J=-2.8$, e a curva $C$ fixa $\mu / J=-2$.

Notamos que ao cruzar a pseudo-transição gás-líquido desordenado, curva $A$, há aumento da densidade com a temperatura, acompanhada de uma diminuição da "magnetização", o que sugere que os lipídios vão para a quase-fase desordenada. Por outro lado, há um máximo na capacidade térmica por sítio para temperatura $k_{B} T / J \approx 1$, mostrando um desacordo com o ponto onde essa quase-fronteira é cruzada, no diagrama (a). Entretanto, como já comentamos, essa linha foi obtida analiticamente a partir das entradas da matriz de transferência e mostra uma pequena diferença com relação ao comportamento real do sistema, como mostra a figura (5.4), sendo levemente deslocada para cima e não traduz o aspecto mais disperso que a verdadeira quase-fronteira apresenta. Na curva $B$, sobre a pseudo-transição gás-líquido de- 
(a)

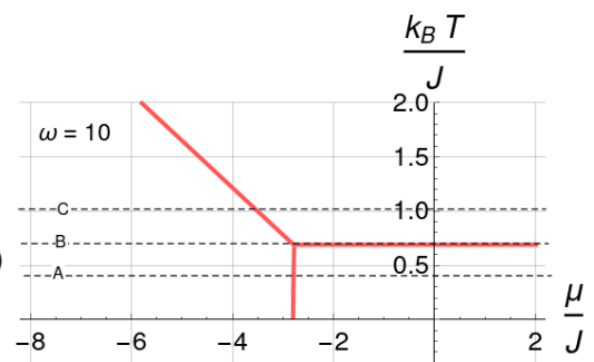

(c)

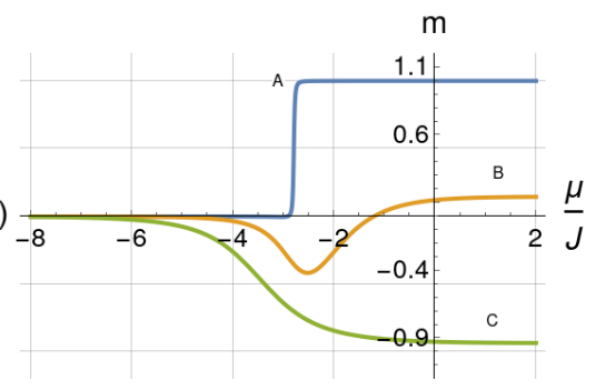

(b)

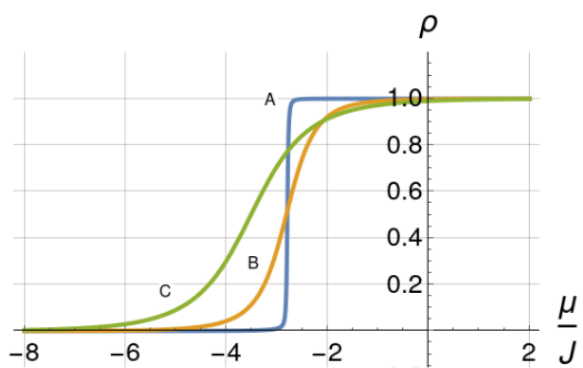

(d)

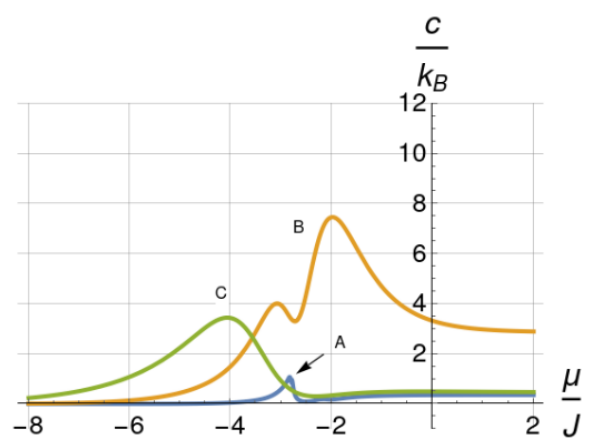

Figura 5.9: Em (a), o diagrama de quase-fases do DLG para degenerescência 10, as linhas horizontais tracejadas $A, B$ e $C$ são os valores de temperatura fixas $k_{B} T / J=0.4,0.68$ e 1 e coincidem com as curvas dos demais gráficos, legendadas com as mesmas letras. Em (b), a densidade em função do potencial químico. Em (c), a "magnetização"por sítio em função do potencial químico. Em (d), a capacidade térmica por sítio a potencial químico constante em função do potencial químico. Todos os gráficos são para $\omega=10$.
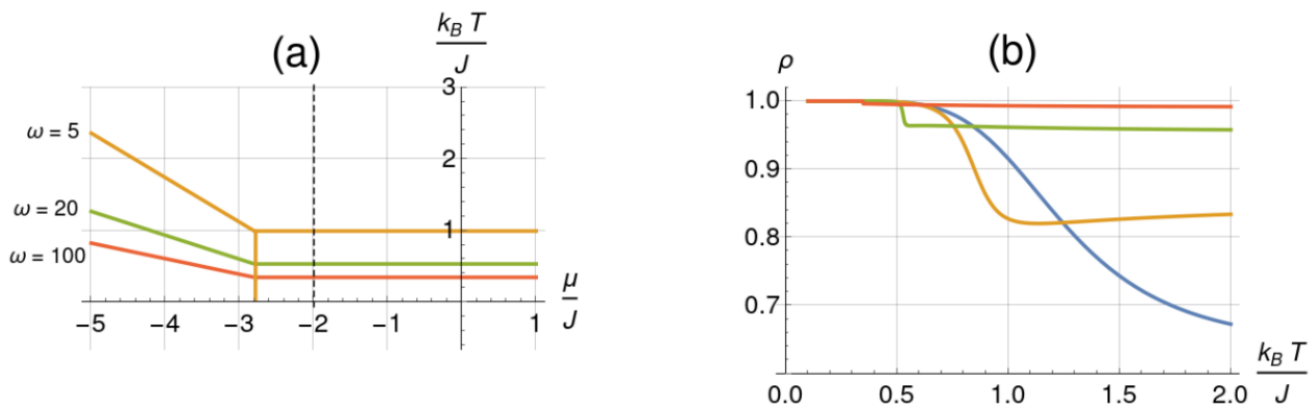

(c)
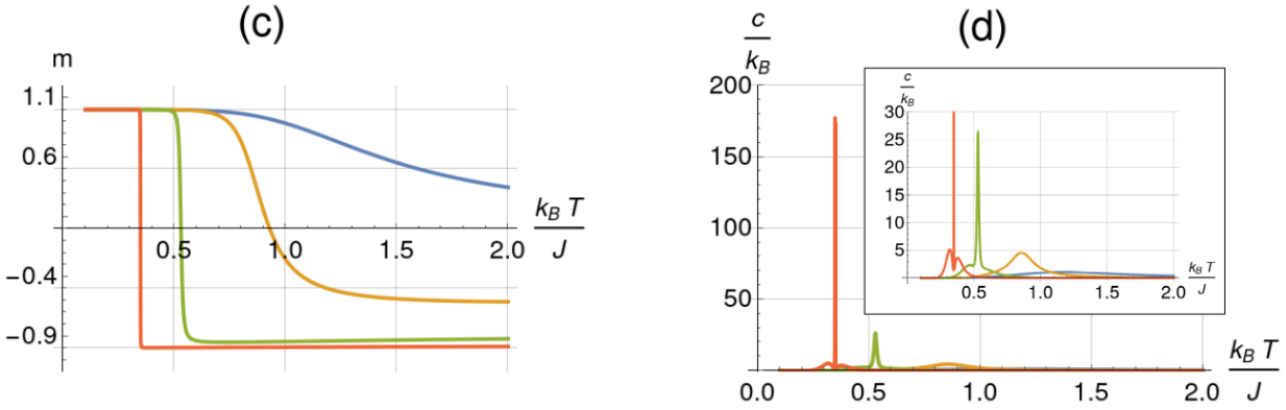

Figura 5.10: Em (a), diagrama de quase-fases do DLG para as degenerescências 5, 20 e 100, as cores das linhas correspondem às degenerescências das curvas nos demais gráficos, a única degenerescência que não aparece no gráfico (a) é $\omega=1$. As curvas azul, amarela, verde e vermelha são para $\omega=1,5,20$ e 100, respectivamente. Em (b), a densidade em função da temperatura. Em (c) a "magnetização"em função da temperatura. Em (d), a capacidade térmica por sítio a potencial químico constante em função da temperatura. 

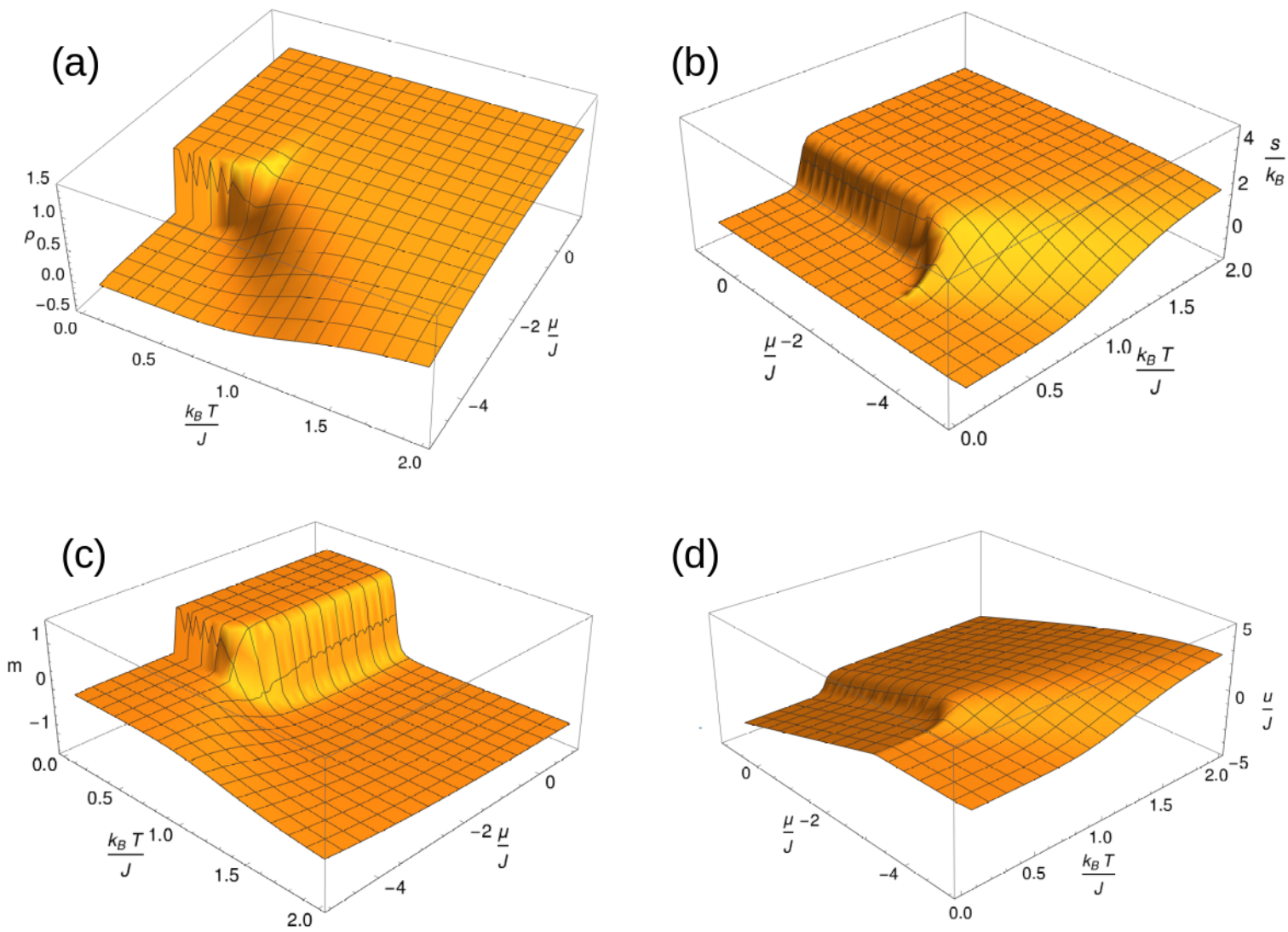

Figura 5.11: Superfícies tridimensionais do fluido de rede de Doniach obtidos para degenerescência 10. Em (a), a densidade. Em (b), a entropia por sítio. Em (c), a "magnetização"por sítio. Em (d), energia interna por sítio. 
sordenado, a rede começa semipreenchida - lembramos que o mesmo ocorre para o fluido de rede quando fixamos $\mu$ sobre a pseudo-transição — , há uma diminuição da densidade seguida de um aumento, bem como uma diminuição da "magnetização", o que sinaliza que o sistema passa rapidamente pela quase-fase gás antes de ir para a quase-fase líquido desordenado, ou em alguma região entre eles. Há também um leve aumento na capacidade térmica por sítio nas proximidades do "ponto triplo". Por fim, na curva $C$, que cruza a linha de pseudo-transição entre as quase-fases líquido ordenado e líquido desordenado, há uma diminuição relativamente abrupta na densidade, seguida de um patamar, e acompanhada de uma diminuição, igualmente repentina, na "magnetização", além de um pico, um pouco mais acentuado do que os outros, na capacidade térmica por sítio, o que sugere que essas quase-fases apresentam um contraste mais marcante do que o existente entre as quase-fases gás e líquido desordenado.

Em seguida, vamos nos debruçar sobre o que ocorre ao sistema sob variação de potencial químico, para valores de temperatura constantes. No gráfico (a) da figura (5.9), a linha tracejada $A$ remete à temperatura $k_{B} T / J=0.4$, a linha $B$ à temperatura $k_{B} T / J=0.68$, que é precisamente a quase-fronteira obtida da relação (5.39) e a linha $C, k_{B} T / J=1$.

Primeiramente, na curva $A$, vemos uma mudança abrupta na densidade e na magnetização, passando de uma rede vazia para uma rede preenchida com lipídios ordenados, acompanhado de um pequeno pico na capacidade térmica por sítio, o que sinaliza uma pseudo-transição gás-líquido ordenado. Na linha $B$, que passa paralelamente sobre a quase-fronteira líquido ordenado-líquido desordenado, há um aumento na densidade. Por outro lado, há uma diminuição da magnetização até atingir um mínimo seguido de um aumento, até um patamar um pouco maior do que zero, o que sugere que o sistema passou rapidamente pelo estado líquido desordenado antes de incidir sobre a pseudo-transição. Curiosamente também é possível observar dois máximos na capacidade térmica por sítio, provavelmente relacionado ao cruzamento da pseudo-transição gás-líquido desordenado e ao ponto onde atingirá a pseudo-transição gáslíquido ordenado. A linha $C$ se comporta de maneira similar à linha $A$ da figura (5.8), ambas cruzam a quase-fronteira gás-liquido desordenado, apresentando mudanças significativas, mas não abruptas, nas propriedades do sistema.

Na figura (5.10), procuramos avaliar o efeito da degenerescência nas pseudo-transições. No 
diagrama (a), vemos que o aumento de $\omega$ força as linhas de pseudo-transição a se aproximarem das regiões de baixa temperatura — ao passo que nada ocorre à pseudo-transição gás-fluido ordenado. Embora não mostremos a linha referente ao sistema não degenerado no diagrama (a), a sua curva está representada pela cor azul nos demais gráficos, observamos que não há mudança abrupta em nenhuma das suas quantidades. Em contrapartida, para $\omega=100$, ao cruzar a quasefronteira, em $\mu / J=0.35$, há uma diminuição pequena, quase imperceptível, porém bastante abrupta, na densidade, simultaneamente vemos uma mudança na "magnetização", semelhante a uma função degrau, bem como um pico acentuado na capacidade térmica por sítio. Curiosamente também aparecem dois pequenos máximos, antes e depois do pico, apesar de ainda não estar claro para nós o motivo desse comportamento, vale o destaque. Novamente, vemos que a temperatura desempenha papel central no fenômeno da pseudo-transição, ao que parece, os vestígios das fases termodinâmicas são mais perceptíveis a baixas temperaturas.

Por fim, com o objetivo de visualizar as diferentes regiões termodinâmicas do modelo, mostramos na figura (5.11) as superfícies tridimensionais para as quantidades analisadas, ainda com $\omega=10$. 


\section{Capítulo 6}

\section{Conclusões e considerações finais}

Neste trabalho, lançamos um olhar sobre modelos simplificados na rede unidimensional baseados no modelo de Ising _ , seguindo concomitantemente dois caminhos: a análise de modelos inspirados em biomembranas artificiais, particularmente o fluido de rede de Doniach (DLG), e o fenômeno das pseudo-transições e quase-fases.

A adição da degenerescência possibilitou observar pseudo-transições no modelo de Ising unidimensional de spin $1 / 2$ sob variação de temperatura, o que não podia ser observado no caso não-degenerado, já que a quase-fronteira está sobre a linha $H=0$. A degenerescência força uma mudança no ângulo dessa linha com relação ao eixo horizontal, permitindo que seja cruzada para um conjunto de valores de campo fixos. A análise também mostrou o papel fundamental da temperatura no contraste entre as quase-fases adjacentes, sendo mais nítido quanto menor é a temperatura onde ocorre a pseudo-transição. Em decorrência disso, o aumento da degenerescência, ao menos no modelo de Ising degenerado e no DLG, promove um aumento no contraste entre as quase-fases, já que aproxima a pseudo-transição da região de baixa temperatura.

O estudo do fluido de rede de Doniach unidimensional — que é um modelo de Ising de spin 1 degenerado —, também sinalizou a existência de pseudo-transições e quase-fases, com características similares àquelas obtidas para os modelos de dois estados. Embora sem uma justificativa rigorosa, verificamos que o método de analisar os elementos da diagonal principal da matriz de transferência nos ajuda a identificar as linhas de pseudo-transição. Uma formalização desse processo pode trazer importantes contribuições no estudo desses sistemas e deixamos 
como possibilidade para trabalhos futuros.

Outra questão em que esbarramos foi a noção de ponto crítico nos modelos unidimensionais, que possuem características de transições de primeira e segunda ordens, simultaneamente. Não encontramos, na literatura, algum texto que se aprofunde no problema, mas acreditamos que seja algo que vale ser investigado. Associado a isso, nos deparamos com um cenário, para a entropia residual crítica, que não foi previsto na conjectura proposta por Rojas. É possível, por exemplo, que essa dualidade na natureza do ponto crítico esteja relacionada com a dualidade nas linhas de pseudo-coexistência, já que ambas possuem características de transições contínuas e descontínuas - sem descontinuidade nas linhas, claro. Talvez uma ampliação da conjectura, acompanhada de uma melhor compreensão do que entendemos por ponto crítico em modelos unidimensionais, contribua no entendimento desses sistemas.

Por fim, nossa análise reforça a afirmação de que modelos unidimensionais podem fornecer pistas importantes sobre o comportamento do sistema em dimensões maiores, o que é corroborado ao confrontar os resultados obtidos aqui com aqueles já conhecidos, como o modelo de Ising e o DLG resolvidos na aproximação de campo médio. 


\section{Referências Bibliográficas}

[1] D. Voet and J. Voet, Biochemistry 1. Wiley \& Sons, 3nd ed., 2004.

[2] T. Ising, R. Folk, R. Kenna, B. Berche, and Y. Holovatch, "The fate of ernst ising and the fate of his model," 2017.

[3] S. Salinas, Introduçăo à Física Estatística. São Paulo: EDUSP, 1997.

[4] R. Baxter, Exactly Solved Models in Statistical Mechanics. London: Academic, 1982.

[5] K. Huang, Statistical Mechanics. New York: John Wiley \& Sons, Inc., 2nd ed., 1987.

[6] L. Onsanger, "Crystal statistics. i. a two-dimensional model with an order-disorder transition," Physical Review, vol. 65, no. 177, 1944.

[7] S. Doniach, "Thermodynamic fluctuations in phospholipid bilayers," The Journal of Chemical Physics, vol. 68, no. 4912, 1978.

[8] H. Guidi, Modelos estatísticos para a transição ordem-desordem de camadas lipídicas. PhD thesis, IFUSP, 2012.

[9] H. Guidi and V. Henriques, "Lattice solution model for order-disorder transitions in membranes and langmuir monolayers," Physical Review, vol. 90, no. 052705, 2014.

[10] L. Landau and E. Lifshitz, Statistical Physics Part 1. New York: Pergamon, 1980.

[11] K. Christensen and N. R. Moloney, Complexity and Criticality. PUBLISHED BY IMPERIAL COLLEGE PRESS AND DISTRIBUTED BY WORLD SCIENTIFIC PUBLISHING CO., 2005. 
[12] C. Kittel, "Phase transition of a molecular zipper," American Journal of Physics, vol. 37, no. $917,1969$.

[13] S. Chui and J. Weeks, "Pinning and roughening of one-dimensional models of interfaces and steps," Physical Review B, vol. 23, no. 2438, 1981.

[14] T. Dauxois, M. Peyrard, and A. R. Bishop, "Entropy-driven transition in a one-dimensional system," Physical Review B, vol. 51, no. 4027, 1995.

[15] P. Sarkanych, Y. Holovatch, and R. Kenna, "Exact solution of a classical short-range spin model with a phase transition in one dimension: The potts model with invisible states," Physics Letters A, vol. 381, no. 41, pp. 3589-3593, 2017.

[16] L. van Hove, "Sur l'intégrale de configuration pour les systèmes de particules À une dimension," Physica, vol. 16, no. 2, pp. 137 - 143, 1950.

[17] J. Cuesta and A. Sánches, "General non-existence theorem for phase transitions in onedimensional systems with short range interactions, and physical examples of such transitions," Journal of Statistical Physics, vol. 115, no. 3, pp. 869-893, 2004.

[18] R. Horn and C. Johnson, Matrix Analysis. Cambridge: Cambridge University Press, 11985.

[19] P. N. Timonin, "Spin ice in a field: Quasi-phases and pseudo-transitions," Journal of Experimental and Theoretical Physics, vol. 113, p. 251-265, Aug 2011.

[20] S. M. d. de Souza and O. Rojas, "Quasi-phases and pseudo-transitions in one-dimensional models with nearest neighbor interactions," Solid State Communications, vol. 269, pp. 131-134, 2018.

[21] O. Rojas, "A conjecture on the relationship between critical residual entropy and finite temperature pseudo-transitions of one-dimensional models," 2020.

[22] I. Carvalho, J. Torrico, S. de Souza, O. Rojas, and O. Derzhko, "Correlation functions for a spin-ising-xyz diamond chain: Further evidence for quasi-phases and pseudo-transitions," Annals of Physics, vol. 402, no. 45, pp. 45-65, 2019. 
[23] O. Rojas, J. Strečka, O. Derzhko, and S. M. de Souza, "Peculiarities in pseudo-transitions of a mixed spin-( $(1 / 2,1)$ ising-heisenberg double-tetrahedral chain in an external magnetic field," Journal of Physics: Condensed Matter, vol. 32, no. 035804, 2020.

[24] I. M. Carvalho, "Funções de correlação para uma cadeia de ising decorada com spins heisenberg numa estrutura diamante," Master's thesis, Universidade Federal de Lavras, Lavras, 2018.

[25] G. Cevc and D. Marsh, Phospholipid Bilayer. John Wiley \& Sons, 1987.

[26] M. Lamy-Freund and K. A. Riske, "The peculiar thermo-structural behavior of the anionic lipid dmpg.," Chemistry and physics of lipids, vol. 122 1-2, pp. 19-32, 2003.

[27] T. Enoki, V. Henriques, and M. Lamy, "Light scattering on the structural characterization of dmpg vesicles along the bilayer anomalous phase transition," Chemistry and Physics of Lipids, vol. 165, no. 8, pp. 826-837, 2012.

[28] R. G. R. Nunes, “Transição de fase ordem-desordem em membranas na presença de dissociação: modelos estatísticos,” 2011.

[29] M. N. Tamashiro, C. Barbetta, R. Germano, and V. B. Henriques, "Phase transitions and spatially ordered counterion association in ionic-lipid membranes: a statistical model," Phys. Rev. E, vol. 84, p. 031909, Sep 2011.

[30] V. B. Henriques, R. Germano, M. T. Lamy, and M. N. Tamashiro, "Phase transitions and spatially ordered counterion association in ionic-lipid membranes: theory versus experiment," Langmuir, vol. 27, no. 21, pp. 13130-13143, 2011.

[31] C. P. B. Vignoto, "Modelagem teórica de monocamadas anfifílicas: gás de rede de doniach em redes bipartidas na aproximação de campo médio,” 2020.

[32] F. O. de Oliveira, "Modelagem teórica de monocamadas anfifílicas: o gás de rede de doniach em aproximação de pares," 2016.

[33] F. O. de Oliveira and M. N. Tamashiro, "Phase transitions in phospholipid monolayers: statistical model at the pair approximation,” Phys. Rev. E, vol. 99, p. 012147, Jan 2019. 
[34] F. O. de Oliveira and M. N. Tamashiro, "Phase transitions in phospholipid monolayers: theory versus experiments," Langmuir, vol. 35, no. 10, pp. 3848-3858, 2019.

[35] H. Callen, Thermodynamics and an introduction to thermostatistics. New York: Wiley, 1985.

[36] M. Oliveira, Termodinâmica. New York: Livraria da Fisica, 2005.

[37] J. Yeomans, Statistical Mechanics of Phase Transitions. Oxford Science Publications, 1993.

[38] W. Kaplan, Cálculo Avançado - Vol. 2. Blucher, 1972.

[39] F. U. Coelho and M. L. Lourenço, Um Curso de Álgebra Linear. EDUSP, 2nd ed., 2013.

[40] B. M.A.A., F. Barbosa, and F. Oliveira, "Thermodynamic and dynamic anomalies in a one-dimensional lattice model of liquid water," The Journal of chemical physics, vol. 2, no. $134,2011$.

[41] E. O. Rizzatti, M. A. A. Barbosa, and M. C. Barbosa, "Quantum density anomaly in optically trapped ultracold gases," Physical Review A, vol. 102, Sep 2020.

[42] B. M.A.A., F. Barbosa, and F. Oliveira, "Residual entropy of ice nanotubes and ice layers," Physica A: Statistical Mechanics and its Applications, vol. 392, no. 4, pp. 680-688, 2013.

[43] M. Blume, V. J. Emery, and R. B. Griffiths, "Ising model for the $\lambda$ transition and phase separation in he ${ }^{3}-\mathrm{he}^{4}$ mixtures," Phys. Rev. A, vol. 4, pp. 1071-1077, Sep 1971.

[44] M. Blume, "Theory of the first-order magnetic phase change in $\mathrm{uo}_{2}$," Phys. Rev., vol. 141, pp. 517-524, Jan 1966.

[45] H. Capel, "On the possibility of first-order phase transitions in ising systems of triplet ions with zero-field splitting," Physica, vol. 32, pp. 966-988, May 1966. 


\section{Apêndice A}

\section{Método da matriz de transferência}

Considere um modelo unidimensional de rede, com $N$ sítios, e uma quantidade finita de estados discretos possíveis. A função de partição do modelo é dada por

$$
Z=\sum_{\{\sigma\}} e^{-\beta \mathcal{H}(\{\sigma\})}=\sum_{\{\sigma\}} \prod_{i=1}^{N} T\left(\sigma_{i}, \sigma_{i+1}\right),
$$

onde $T\left(\sigma_{i}, \sigma_{i+1}\right)$ são os elementos da matriz de transferência $\mathbb{T}$. Vamos mostrar que a equação acima expressa o traço da matriz $\mathbb{T}^{N}$.

Primeiro, note que se temos duas matrizes $\mathbb{A}$ e $\mathbb{B}$, o produto $\mathbb{C}=\mathbb{A} \cdot \mathbb{B}$ entre elas possui elementos dados por

$$
C_{i j}=\sum_{k} A_{i k} B_{k j}
$$

Logo, ao multiplicar $A$ por ela mesma, temos

$$
\begin{gathered}
A_{i j}^{2}=\sum_{k} A_{i k} A_{k j}, \\
A_{i j}^{3}=\sum_{k} A_{i k}^{2} A_{k j}=\sum_{k}\left(\sum_{l} A_{i l} A_{l k}\right) A_{k j}=\sum_{k, l} A_{i l} A_{l k} A_{k j},
\end{gathered}
$$




$$
A_{i j}^{4}=\sum_{k} A_{i k}^{2} A_{k j}^{2}=\sum_{k}\left(\sum_{l} A_{i l} A_{l k}\right)\left(\sum_{n} A_{k n} A_{n j}\right)=\sum_{k, l, n} A_{i l} A_{l k} A_{k n} A_{n j},
$$

e assim sucessivamente. Observe que a estrutura de (A.1) é idêntica a desse produto quando assumimos condições de contorno periódicas, isto é, quando $\sigma_{N+1}=\sigma_{1}$. Com efeito

$$
\begin{gathered}
Z=\sum_{\sigma_{1}} \sum_{\sigma_{2}} \ldots \sum_{\sigma_{N}} T\left(\sigma_{1}, \sigma_{2}\right) T\left(\sigma_{2}, \sigma_{3}\right) \ldots T\left(\sigma_{N-1}, \sigma_{N}\right) T\left(\sigma_{N}, \sigma_{N+1}\right) \\
=\sum_{\sigma_{1}} \sum_{\sigma_{3}} \ldots \sum_{\sigma_{N}}[\underbrace{\sum_{\sigma_{2}} T\left(\sigma_{1}, \sigma_{2}\right) T\left(\sigma_{2}, \sigma_{3}\right)}_{T^{2}\left(\sigma_{1}, \sigma_{3}\right)}] T\left(\sigma_{3}, \sigma_{4}\right) \ldots T\left(\sigma_{N-1}, \sigma_{N}\right) T\left(\sigma_{N}, \sigma_{N+1}\right) \\
=\sum_{\sigma_{1}} \sum_{\sigma_{4}} \ldots \sum_{\sigma_{N}}[\underbrace{\sum_{\sigma_{3}} T^{2}\left(\sigma_{1}, \sigma_{3}\right) T\left(\sigma_{3}, \sigma_{4}\right)}_{T^{3}\left(\sigma_{1}, \sigma_{4}\right)}] T\left(\sigma_{4}, \sigma_{5}\right) \ldots T\left(\sigma_{N-1}, \sigma_{N}\right) T\left(\sigma_{N}, \sigma_{N+1}\right) \\
\vdots \\
Z=\sum_{\sigma_{1}} \sum_{\sigma_{N}} T^{N+1}\left(\sigma_{1}, \sigma_{N}\right) T\left(\sigma_{N}, \sigma_{N+1}\right)=\sum_{\sigma_{1}} T^{N}\left(\sigma_{1}, \sigma_{N+1}\right) \stackrel{\sigma_{N+1}=\sigma_{1}}{=} \sum_{\sigma_{1}} T^{N}\left(\sigma_{1}, \sigma_{1}\right) .
\end{gathered}
$$

A expressão acima representa o traço da matriz de transferência elevada à $N$-ésima potência. Finalmente, podemos reescrever (A.1) como

$$
Z=\operatorname{tr}\left(\mathbb{T}^{N}\right)
$$


Agora, note que a matriz de transferência é real e simétrica, o que garante que ela é diagonalizável por uma matriz invertível $\mathbb{P}$ [39]. Além disso, o traço de uma matriz é invariante por mudança de base, de modo que a função de partição pode ser calculada a partir dos autovalores $\lambda_{k}$ de $\mathbb{T}$, que também não se alteram por mudança de base. De fato

$$
\operatorname{tr}(\mathbb{V})=\operatorname{tr}\left(\mathbb{P}^{-1} \mathbb{T P}\right)=\operatorname{tr}\left(\mathbb{T} \mathbb{P} \mathbb{P}^{-1}\right)=\operatorname{tr}(\mathbb{T})
$$

Assim, para um sistema com $n$ estados possíveis

$$
\mathbb{V}=\mathbb{P}^{-1} \mathbb{T P}=\left(\begin{array}{cccc}
\lambda_{1} & 0 & \ldots & 0 \\
0 & \lambda_{2} & \ldots & 0 \\
\vdots & \vdots & \ddots & 0 \\
0 & 0 & 0 & \lambda_{n}
\end{array}\right)
$$

onde $\lambda_{k}, k=1, \ldots, n$, são os autovalores associados a $\mathbb{T}$. Também é fácil verificar que

$$
\mathbb{V}^{N}=\left(\begin{array}{cccc}
\lambda_{1}^{N} & 0 & \ldots & 0 \\
0 & \lambda_{2}^{N} & \ldots & 0 \\
\vdots & \vdots & \ddots & 0 \\
0 & 0 & 0 & \lambda_{n}^{N}
\end{array}\right)
$$

Por fim, a função de partição é

$$
Z=\sum_{i=1}^{N} \lambda_{i}^{N},
$$

cujos autovalores são dados pelas raízes do polinômio característico de $\mathbb{T}$

$$
p(\lambda)=\operatorname{det}[\mathbb{T}-\lambda \mathbb{I}]
$$




\section{Apêndice B}

\section{Resolução da equação cúbica}

Considere a equação cúbica

$$
\lambda^{3}+a \lambda^{2}+b \lambda+c=0
$$

Primeiro vamos simplificar a equação eliminando o termo quadrático, tome

$$
\lambda=x+m,
$$

de modo que

$$
\begin{gathered}
(x+m)^{3}+a(x+m)^{2}+b(x+m)+c=0 \\
\left(x^{3}+3 x^{2} m+3 x m^{2}+m^{3}\right)+a\left(x^{2}+2 x m+m^{2}\right)+b(x+m)+c=0 \\
x^{3}+(3 m+a) x^{2}+\left(3 m^{2}+2 a m+b\right) x+\left(m^{3}+a m^{2}+b m+c\right)=0,
\end{gathered}
$$

impondo

$$
3 m+a=0 \Longrightarrow m=-\frac{a}{3}
$$

a solução assume a forma

$$
\lambda=x-\frac{a}{3}
$$


e a equação (B.2) se torna

$$
\begin{gathered}
x^{3}+\left[3\left(-\frac{a}{3}\right)^{2}+2 a\left(-\frac{a}{3}\right)+b\right] x+\left[\left(-\frac{a}{3}\right)^{3}+a\left(-\frac{a}{3}\right)^{2}+b\left(-\frac{a}{3}\right)+c\right]=0 \Longrightarrow \\
x^{3}+\left(\frac{-a^{2}+3 b}{3}\right) x+\left(\frac{2 a^{3}-9 a b+27 c}{27}\right)=0 .
\end{gathered}
$$

Agora vamos definir alguns parâmetros

$$
\Delta_{0}:=a^{2}-3 b, \quad \Delta_{1}:=-2 a^{3}+9 a b-27 c, \quad p:=-\frac{\Delta_{0}}{3}, \quad q:=-\frac{\Delta_{1}}{27} .
$$

de modo que a equação reduzida é

$$
x^{3}+p x+q=0
$$

Fazendo uma nova mudança de variáveis através de

$$
x=u+\frac{n}{u}
$$

temos

$$
\begin{gathered}
\left(u+\frac{n}{u}\right)^{3}+p\left(u+\frac{n}{u}\right)+q=0, \\
u^{3}+(3 n+p)\left(u+\frac{n}{u}\right)+\frac{n^{3}}{u^{3}}+q=0 .
\end{gathered}
$$

Para que o termo cruzado desapareça, fazemos uma nova imposição

$$
3 n+p=0 \quad \Longrightarrow \quad n=-\frac{p}{3} .
$$

Logo 


$$
x=u-\frac{p}{3 u},
$$

de forma que a equação (B.6) se torna

$$
\begin{aligned}
& u^{3}+q-\frac{p^{3}}{27 u^{3}}=0, \\
& v^{2}+q v-\frac{p^{3}}{27}=0 .
\end{aligned}
$$

Veja que se trata de uma equação do segundo grau em $v=u^{3}$. Com um pouco de trabalho algébrico não é difícil mostrar que as raízes são dadas por

$$
u_{ \pm}=\sqrt[3]{v_{ \pm}}=\frac{1}{3}\left(\frac{\Delta_{1} \pm \sqrt{\Delta_{1}^{2}-4 \Delta_{0}^{3}}}{2}\right)^{\frac{1}{3}}
$$

Entretanto, de forma geral, raízes cúbicas possuem 3 soluções complexas

$$
u_{ \pm, 0}=\sqrt[3]{v_{ \pm}}, \quad u_{ \pm, 1}=\sqrt[3]{v_{ \pm}} e^{i \frac{2 \pi}{3}}, \quad u_{ \pm, 2}=\sqrt[3]{v_{ \pm}} e^{i \frac{4 \pi}{3}}
$$

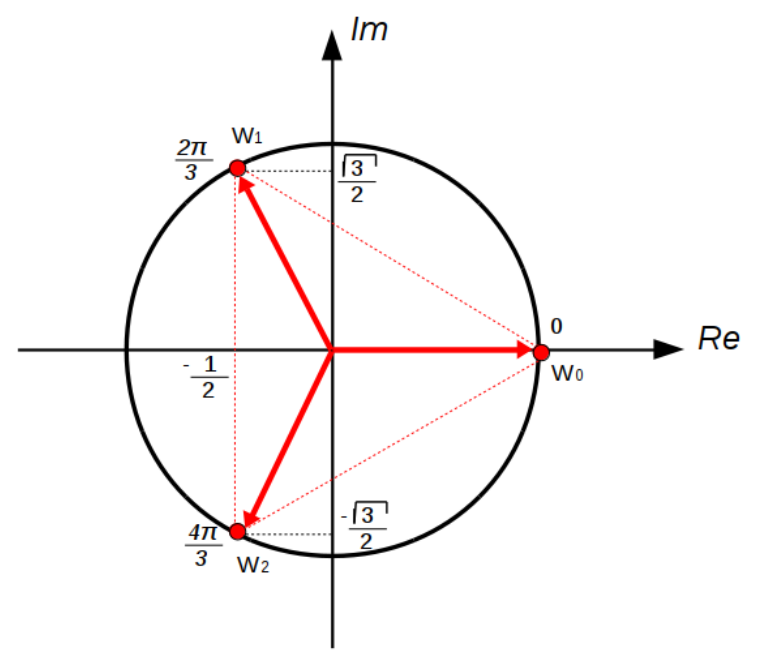

Figura B.1: Raízes cúbicas de 1.

Para determinar as soluções (B.7), considere a conhecida propriedade das raízes de equações de segundo grau para (B.8) 


$$
\begin{gathered}
v_{+} \cdot v_{-}=-\frac{p^{3}}{27} \Longrightarrow u_{+} \cdot u_{-}=-\frac{p}{3}, \\
u_{ \pm}=-\frac{p}{3 u_{\mp}},
\end{gathered}
$$

de maneira que, retornando a $x$, obtemos

$$
\begin{gathered}
x_{0}=u_{+, 0}-\frac{p}{3 u_{+, 0}}=u_{+, 0}+u_{-, 0} \quad \Longrightarrow \quad x_{0}=\sqrt[3]{v_{+}}+\sqrt[3]{v_{-}}, \\
x_{1}=u_{+, 1}-\frac{p}{3 u_{+, 1}}=\sqrt[3]{v_{+}} e^{i \frac{2 \pi}{3}}-\frac{p}{3 \sqrt[3]{v_{+}} e^{i \frac{2 \pi}{3}}} \quad \Longrightarrow \quad x_{1}=\sqrt[3]{v_{+}} e^{i \frac{2 \pi}{3}}+\sqrt[3]{v_{-}} e^{-i \frac{2 \pi}{3}}, \\
x_{2}=u_{+, 2}-\frac{p}{3 u_{+, 2}}=\sqrt[3]{v_{+}} e^{i \frac{4 \pi}{3}}-\frac{p}{3 \sqrt[3]{v_{+}} e^{i \frac{4 \pi}{3}}} \quad \Longrightarrow \quad x_{2}=\sqrt[3]{v_{+}} e^{i \frac{4 \pi}{3}}+\sqrt[3]{v_{-}} e^{-i \frac{4 \pi}{3}} .
\end{gathered}
$$

Finalmente, de forma mais compacta, a solução geral (B.3) é dada por

$$
\lambda_{k}=-\frac{a}{3}+\sqrt[3]{v_{+}} e^{i \frac{2 k \pi}{3}}+\sqrt[3]{v_{-}} e^{-i \frac{2 k \pi}{3}} ; \quad k=0,1,2 .
$$

Para finalizar, na expressão (B.9) note que quando $\Delta_{1}^{2}-4 \Delta_{0}^{3}<0$, temos

$$
v_{ \pm}=\frac{1}{3}\left(\frac{\Delta_{1} \pm i \sqrt{4 \Delta_{0}^{3}-\Delta_{1}^{2}}}{2}\right)^{\frac{1}{3}}=\frac{1}{3}\left(\left|\Delta_{0}\right|^{\frac{3}{2}} e^{ \pm i \theta}\right)^{\frac{1}{3}}
$$

onde

$$
\theta=\tan ^{-1}\left(\frac{\sqrt{4 \Delta_{0}^{3}-\Delta_{1}^{2}}}{\Delta_{1}}\right)
$$

nos fornecendo

$$
u_{ \pm}=\frac{\sqrt{\Delta_{0}} e^{ \pm i \frac{\theta}{3}}}{3} .
$$


Nesse caso, (B.3) se torna

$$
\begin{gathered}
\lambda_{k}=-\frac{a}{3}+\frac{\sqrt{\Delta_{0}} e^{i \frac{\theta}{3}}}{3} e^{i \frac{2 k \pi}{3}}+\frac{\sqrt{\Delta_{0}} e^{-i \frac{\theta}{3}}}{3} e^{-i \frac{2 k \pi}{3}}=-\frac{a}{3}+\frac{\sqrt[3]{\Delta_{0}}}{3}\left[e^{i\left(\frac{\theta+2 k \pi}{3}\right)}+e^{-i\left(\frac{\theta+2 k \pi}{3}\right)}\right], \\
\lambda_{k}=-\frac{a}{3}+\frac{2 \sqrt[3]{\Delta_{0}}}{3} \cos \left(\frac{\theta+2 k \pi}{3}\right) ; k=0,1,2
\end{gathered}
$$

que são precisamente as nossas raízes reais, obtidas da condição $\Delta:=4 \Delta_{0}^{3}-\Delta_{1}^{2}>0$. 